CDD: 511.3

\title{
PROLEGÓMENOS PARA UNA TEORÍA FORMAL DE ESTRUCTURAS (DESPUÉS DE N.DA COSTA ).
}

\section{VIDAL HUMBERTO NAVARRO MENA}

Departamento de Matemática y Cs. de la Computación Universidad de Santiago de Chile, Las Sophoras \# 175. Santiago, Chile.

Departamento de Ciencias de la Computación, Beuchef \# 851. Universidad de Chile ${ }^{1}$, Santiago, Chile.

vidal.navarro@usach.cl

Received: 22.04.2015; Accepted: 24.05.2015

Abstract: The present article has for objective to present an alternative reconstruction of Concept of Structure, motivated by the articles [1] and [3], as an abstract generalization of what is a mathematical object. First, its construction is presented, that is related to the Type Theory and Order in Logic. Which give us a context for properties and several interesting examples. Secondly, we will proceed towards a concrete semantic for analysing structures and letting us operate in them. Thus we are able to know "what itXB is true in it". Results of order and individuals reduction are presented through our construction. In the end, we formalized the discussions referred completely in our Type Theory.

keywords: Structure, Models, Structure Semantics, High-Order Logic, Newton Da Costa.

\footnotetext{
${ }^{1}$ Dirección actual.
}

Manuscrito - Rev. Int. Fil., Campinas, v. 38, n.2, pp.89-154, jul.-ago. 2015. 
Resumen: El articulo tiene por objetivo la reconstrucción alternativa del concepto de estructura, motivado por los articulos [1] y [3], como una generalización abstracta de lo que es un objeto matemático. Primero, mostramos su construcción, que tiene que ver con la teoría de tipos y orden en lógica, dando a lugar a propiedades y varios ejemplos interesantes. Luego avanzamos hacia una semántica concreta, para su análisis, y para permitirnos operar sobre ellas, sabiendo de este modo, lo que es "lo verdadero en ella". Obtenido ello, mostraremos los resultados de reducción de orden y de individuos, pero vistos en este contexto, así formalizando completamente en nuestra teoría de tipos la discusión de [1] (Ver también [2] y [3]) sobre estos temas.

Palabras clave: Estructuras, Modelos, Semántica de Estructuras, Lógica de orden mayor, Newton Da Costa.

\section{Índice}

Introducción

1. Reconstrucción del concepto de estructuras

2. Ejemplos de estructuras 21

Estructura simple y múltiple . . . . . . . . . . . . . . . . 21

Estructura análoga . . . . . . . . . . . . . 23

Estructura I fundamental . . . . . . . . . . . . . 31

Estructura completa . . . . . . . . . . 36

3. Lenguajes y semántica para estructuras. 37

Lenguajes formales para tipos. . . . . . . . . . . . 37

Semántica para estructuras. . . . . . . . . . . . . . 42

Transiciones entre estructuras. . . . . . . . . . . . 46

$\begin{array}{ll}\text { Referencias } & 69\end{array}$

Manuscrito - Rev. Int. Fil., Campinas, v. 38, n.2, pp.89-154, jul.-ago. 2015. 


\section{Introducción}

El objetivo del presente trabajo es el estudio de Estructuras motivado substancialmente por [1]. En principio este artículo puede ser leído independientemente de [1], sin embargo la motivación real, y, el extremo detalle con el que se ha hecho este articulo, se aprecia mejor por su conexión con el trabajo de N. da Costa y sus coautores. Destacamos que la motivación de los autores de [1], [2], [3], es explicar desde un punto de vista contemporáneo las investigaciones de J.Sebastiao da Silva y N.krasner sobre una teoría de Galois generalizada. Más que tratar de generalizar la teoría de Galois clásica [5] a otro tipo de ecuaciones ${ }^{2}$ o otras teorías algebraicas ${ }^{3}$ nosotros entendemos la teoría de S. da Silva ${ }^{4}$ como un intento de producir una teoría análoga en lógica matemática. Puede ser importante en este punto citar a A.Robinson: "the suggestion that numerous important concepts of Algebra possess natural geralizations the framework of the Theory of Models has met with a rather less lively response. Nevertheless, it is still the author's belief that investigations in this direction are both interesting and valuable."[8] pagina VI.

En este articulo nos ocuparemos de formalizar completamente la noción de estructura a là N.da Costa y sus coautores, y dejaremos nuestra versión de "teoria de Galois" para otra publicación. Una primera versión de esta teoría ha aparecido en [7]. Empezaremos con la construcción de estructuras y a medida que se avance abarcaremos su semántica. Para ello en el primer capítulo se mostrará la base para el concepto de estructura. Conectándolo con lo mostrado por Newton Da Costa en [1]. En el segundo capítulo continuaremos con ejemplos de estructuras: la estructura simple y múltiple, como la expresión en las estructuras de la diferencia e tipos con un solo individuo y varios, la estructura análoga como herramienta de reducción de individuos, la estructura I fundamental como herramienta de reducción de orden, y por ultimo la estructura completa, como formalización del objeto $\varepsilon(D)$, en [1] pagina 4, que interesantemente, no es estrictamente una estructura

\footnotetext{
${ }^{2}$ Como lo hizo S.Lie y definitivamente Vessiot para ecuaciones diferenciales.

${ }^{3}$ Como en el caso de Krasner

${ }^{4}$ Como explicada en [1].
}

Manuscrito - Rev. Int. Fil., Campinas, v. 38, n.2, pp.89-154, jul.-ago. 2015. 
en el sentido dado aquí. En el tercer capítulo construimos una semántica pertinente para ello reconstruimos los lenguajes, con la salvedad de que ahora son para varios individuos. A partir de ello formalizaremos las herramientas para la interpretación, y así avanzar hacia una teoría de modelos para estructuras. Habiendo desarrollado estas herramientas esenciales, mostraremos un concepto bastante intuitivo ${ }^{5}$, al que llamaremos transición. Sumado a las herramientas mencionadas, posibilitará una demostración de nuestros principales teoremas, reducción de individuos (3.19), reducción de orden (3.22) y corolario sobre reducción de orden e individuos (3.23).

\section{Reconstrucción del concepto de estruc- turas}

Lo a realizar en este trabajo sera bajo la teoría de conjuntos de Neuman-Bernays-Gödel(NBG), aunque la exposición sea un tanto informal, esta sera rigurosa y precisa, en el sentido usual de los trabajos de lógica matemática: usamos argumentos de matemática estándar en el entendimiento que todo se puede formalizar en el lenguaje adecuado.

Definición 1.1. Sea un conjunto $I \neq \emptyset$ definimos:

1. El generado de I, como el conjunto

$$
\mathcal{G}(I)=\left\{\left\langle a_{1}, \ldots, a_{n}\right\rangle \mid a_{1}, \ldots, a_{n} \in I \wedge 1 \leq n<\omega\right\},
$$

donde $\omega$ es el menor ordinal transfinito. Además definimos de manera recursiva, para $0 \leq l<\omega$,

$$
\mathcal{G}^{(0)}(I)=I, y, \mathcal{G}^{(l)}(I)=\mathcal{G}\left(\bigcup\left\{G^{(j)}(I) \mid 0 \leq j<l\right\}\right) .
$$

2. El conjunto $T_{I}$, de la forma

$$
T_{I}=\bigcup\left\{\mathcal{G}^{(l)}(I) \mid 0 \leq l<\omega\right\},
$$

Diremos que el conjunto $T_{I}$, que satisface $I \cap \mathcal{G}^{(l)}(I)=\emptyset$, para cualquier $l \in \mathbb{N}$, es el conjunto de tipos de $I$.

\footnotetext{
${ }^{5}$ Claramente visto en otros ámbitos, por ejemplo teoría de modelos saturada, pero de un modo distinto.
}

Manuscrito - Rev. Int. Fil., Campinas, v. 38, n.2, pp.89-154, jul.-ago. 2015. 
3. Los conjuntos $T^{(l)}(I)$, con $0 \leq l<\omega$,

$$
T^{(0)}(I)=\mathcal{G}^{(0)}(I), \quad y, T^{(l)}(I)=\mathcal{G}^{(l)}(I) \backslash \mathcal{G}^{(l-1)}(I) .
$$

Diremos que $T^{(l)}(I)$ es el conjunto de tipos de orden l de $T_{I}$, y llamaremos a $T^{(0)}(I)$ el conjunto de individuos de $T_{I}$.

Notemos que esta definición es equivalente a la definición de los tipos $T$ en [1] página 4. Para ello observemos que en el caso $I=\{i\}$, $a \in T$ de orden ${ }^{6} 0$ entonces $a \in I \subset T_{I}$, luego usando la forma inductiva de $T$ tenemos que $a=\left\langle a_{1}, \ldots, a_{n}\right\rangle \in T$, y usando hipótesis de inducción sobre el orden de $a_{i}$ para $1 \leq i \leq m$, tenemos $a_{i} \in T_{I}$. Luego $a_{i} \in \mathcal{G}^{n_{i}}(I)$ para ciertos $0 \leq n_{i}<\omega$. Así, por la definición anterior, basta fijar $q$ como el máximo de los $n_{i}$, para obtener que

$$
\left\langle a_{1}, \ldots, a_{n}\right\rangle \in \mathcal{G}^{(q+1)}(I) \subset T_{I} .
$$

Así $T \subset T_{I}$. Por otro otro lado es un tanto fácil ver que $T_{I} \subset T$, para ello ver que $\mathcal{G}^{(0)}(I) \subset T$ y que si $\mathcal{G}^{(q)}(I) \subset T$ para cualquier $q<l<\omega$ entonces $\mathcal{G}^{(l)}(I) \subset T$. Así, como $l$ es arbitrario, tenemos $T_{I} \subset T$.

Proposición 1.2. Sea $1 \leq l<\omega$. Tenemos:

1. $\mathcal{G}^{(l)}(I)$ es un conjunto.

2. La clase $\left\{\mathcal{G}^{(j)}(I) \mid 0 \leq j<\omega\right\}$ es un conjunto.

3. Para $1 \leq l \leq l^{\prime}<\omega, \mathcal{G}^{(l)}(I) \subset \mathcal{G}^{\left(l^{\prime}\right)}(I)$.

4. No siempre se cumple que $I \cap \mathcal{G}^{(1)}(I)=\emptyset$, y por lo tanto la restricción hecha en la parte 2 de definición 1.1 tiene sentido para un conjunto arbitrario.

5. $T^{(l)}(I)$ es un conjunto.

6. Los conjuntos $\mathcal{G}^{(l)}(I)$, y, $T^{(l)}(I)$, son distintos del vacío.

7. Sean $0 \leq i \leq j<\omega$, se cumple: $T^{(i)}(I) \cap T^{(j)}(I)=\emptyset$, si y solo si, $i \neq j$.

8. $T_{I}$ es un conjunto y $T_{I}=\bigcup\left\{T^{(j)}(I) \mid 1 \leq j<\omega\right\}$.

\footnotetext{
${ }^{6}$ En el sentido que se le da al "orden" en el trabajo [1] pagina 4.
} 
Demostración. Enumerando las demostraciones:

1. Si $I$ es un conjunto entonces $\mathcal{G}^{(1)}(I)$ considerado como las funciones desde un subconjunto de $\mathbb{N}$ a $I$, es decir, $x \in \mathcal{G}^{(1)}(I)$ entonces $x \in \mathbb{P}(\mathbb{N} \times I)$, donde $\mathbb{P}$ y $\times$ son símbolos para el conjunto potencia y el producto cartesiano, respectivamente. Así $\mathcal{G}^{(1)}(I) \subset \mathbb{P}(\mathbb{N} \times I)$, de lo que se deduce que $\mathcal{G}^{(1)}(I)$ es un conjunto $^{7}$. Por otro lado veamos que para $1 \leq l<\omega$, tenemos que: $x \in \mathcal{G}^{(l)}(I)$, entonces $x \subset \mathbb{N}^{l} \times \mathcal{G}^{(l-1)}(I)$. Usando una maquinada hipótesis de inducción tenemos $\mathcal{G}^{(l-1)}(I)$ es un conjunto, así, $x \in$ $\mathbb{P}\left(\mathbb{N}^{l} \times \mathcal{G}^{(l-1)}(I)\right)$, de lo que se deduce $\mathcal{G}^{(l)}(I) \subset \mathbb{P}\left(\mathbb{N}^{l} \times \mathcal{G}^{(l-1)}(I)\right)$. De la misma forma en que se deduce que $\mathcal{G}^{(1)}(I)$ es un conjunto, deducimos $\mathcal{G}^{(l)}(I)$, para $1 \leq l<\omega$.

2. Notemos que la clase $\left\{\mathcal{G}^{(j)}(I) \mid 0 \leq j<\omega\right\}$ es un conjunto, puesto que claramente hay una aplicación sobreyectiva de $\mathbb{N}^{0}$ a tal ${ }^{8}$.

3. Si $A \subset B$ entonces claramente, por definición anterior, $\mathcal{G}(A) \subset$ $\mathcal{G}(B)$. Además veamos que

$$
\bigcup\left\{\mathcal{G}^{(j)}(I) \mid 0 \leq j<l\right\} \subset \bigcup\left\{\mathcal{G}^{(j)}(I) \mid 0 \leq j<l^{\prime}\right\},
$$

para $l, l^{\prime}$ definidos para este ítem. Ahora usando lo mostrado en un principio

$$
\mathcal{G}\left(\bigcup\left\{\mathcal{G}^{(j)}(I) \mid 0 \leq j<l\right\}\right) \subset \mathcal{G}\left(\bigcup\left\{\mathcal{G}^{(j)}(I) \mid 0 \leq j<l^{\prime}\right\}\right),
$$

claramente tenemos que $\mathcal{G}^{(l)}(I) \subset \mathcal{G}^{\left(l^{\prime}\right)}(I)$, para $1 \leq l \leq l^{\prime}$.

4. Escojamos $I=\left\{i_{1}, i_{2}, f_{I}\right\}$, con $f_{I}:\{1,2\} \mapsto\left\{i_{1}, i_{2}\right\}$, de modo que $f_{I}(j)=i_{j}$, en tal caso, $f_{I}=\left\langle i_{1}, i_{2}\right\rangle$. Luego claramente $f_{I} \in$ $\mathcal{G}^{(1)}(I)$, así, $I \cap \mathcal{G}^{(1)}(I) \neq \emptyset$.

5. Claramente $T^{(l)}(I) \subset \mathcal{G}^{(l)}(I)$, luego por el ítem 1 de esta proposición $T^{(l)}(I)$ es un conjunto ${ }^{9}$.

\footnotetext{
${ }^{7}$ Para mas detalle ver página 234, teorema 8.8 del libro [4].

${ }^{8}$ Para mas detalle ver página 234, teorema 8.7 del libro [4].

${ }^{9}$ Para mas detalle ver página 234 , teorema 8.8 del libro [4].
} 
6. Notemos que $\mathcal{G}^{(0)}(I)=I \neq \emptyset$. Por otro lado, por definición 1.1 ítem 1 ,

$$
\mathcal{G}^{(l+1)}(I)=\mathcal{G}\left(\bigcup\left\{\mathcal{G}^{(j)}(I) \mid 1 \leq j<l+1\right\}\right),
$$

por hipótesis de inducción tenemos que $\mathcal{G}^{(l)}(I) \neq \emptyset$, así, por definición 1.1 ítem 1 tenemos que existe $a \in \mathcal{G}^{(l)}(I)$, de modo que $\langle a\rangle \in \mathcal{G}^{(l+1)}(I)$. Así $\mathcal{G}^{(l+1)}(I) \neq \emptyset$. Para demostrar que $T^{(l)}(I)$ no es vacío notemos algunos hechos:

a) Si $x \notin I$, entonces $\langle x\rangle \notin \mathcal{G}^{(1)}(I)$. Este hecho esta dado por la definición 1.1 ítem 1 .

b) Del mismo modo, deducimos, por la definición 1.1 ítem 2, $x \notin \bigcup\left\{\mathcal{G}^{(j)}(I) \mid 0 \leq j<l\right\}$, entonces $\langle x\rangle \notin \mathcal{G}^{(l)}(I)$.

c) Notemos que por definición $1.1 I \cap \mathcal{G}^{(1)}(I)=\emptyset$, ya que $\mathcal{G}^{(1)}(I) \neq \emptyset$, entonces existe $x \in \mathcal{G}^{(1)}(I)$, de modo que $x \notin I$, luego $T^{(1)}(I) \neq \emptyset$.

Usando como hipótesis de inducción $T^{(l)}(I) \neq \emptyset$, para $1 \leq l<\omega$, obtenemos que: existe $x \in \mathcal{G}^{(l)}(I)$, de modo que $\left.x \notin \mathcal{G}^{(l)}-1\right)(I)$. Ahora, de la definición 1.1 ítem 2 y el ítem 3 de esta proposición, $x \notin \bigcup\left\{\mathcal{G}^{(j)}(I) \mid 0 \leq j<l\right\}$. Luego por el ítem $\left.b\right)$, tenemos $\langle x\rangle \notin$ $\mathcal{G}^{(l)}(I)$, pero a su vez, por definición $1.1\langle x\rangle \in \mathcal{G}^{(l+1)}(I)$. Por lo tanto $T^{(l+1)}(I)=\mathcal{G}^{(l+1)}(I) \backslash \mathcal{G}^{(l)}(I) \neq \emptyset$.

En síntesis $T^{(l)}(I) \neq \emptyset$. Por cierto, $T^{(0)}(I)$ es claramente distinto del vacío dada la definición 1.1.

7. De derecha a izquierda. Si $i \neq j$, entonces $i<j$, luego, por definición 1.1 ítem 2 ,

$$
T^{(i)}(I) \subset \mathcal{G}^{(i)}(I) \subset \mathcal{G}^{(j-1)}(I),
$$

así por definición 1.1 ítem $3 T^{(j)}(I) \cap T^{(i)}(I)=\emptyset$.

De izquierda a derecha, supongamos que $i=j$, y, $T^{(j)}(I) \cap$ $T^{(i)}(I)=\emptyset$, luego tenemos un claro contrasentido con el ítem anterior y la definición 1.1.

8. Por el ítem 2 de esta proposición $T_{I}$ es un conjunto. Veamos que $T^{(0)}(I)=\mathcal{G}^{(0)}(I)$. Ahora supondremos que para $1 \leq l<\omega$,

$$
\bigcup\left\{\mathcal{G}^{(j)}(I) \mid 0 \leq j<l\right\} \subset \bigcup\left\{T^{(j)}(I) \mid 0 \leq j<l\right\},
$$

Manuscrito - Rev. Int. Fil., Campinas, v. 38, n.2, pp.89-154, jul.-ago. 2015. 
luego

$\bigcup\left\{\mathcal{G}^{(j)}(I) \mid 0 \leq j<l\right\} \cup \mathcal{G}^{(l)}(I)=\bigcup\left\{T^{(j)}(I) \mid 0 \leq j<l\right\} \cup \mathcal{G}^{(l)}(I)$,

Como

$\mathcal{G}^{(l-1)}(I) \subset \bigcup\left\{\mathcal{G}^{(j)}(I) \mid 0 \leq j<l\right\}, \mathrm{y}, T^{(l)}(I) \cup \mathcal{G}^{(l-1)}(I)=\mathcal{G}^{(l)}(I)$,

deducimos

$$
\bigcup\left\{T^{(j)}(I) \mid 0 \leq j<l\right\} \cup T^{(l)}(I)=\bigcup\left\{T^{(j)}(I) \mid 0 \leq j<l+1\right\},
$$

Por ultimo, como

$$
\bigcup\left\{\mathcal{G}^{(j)}(I) \mid 0 \leq j<l+1\right\}=\bigcup\left\{\mathcal{G}^{(j)}(I) \mid 0 \leq j<l\right\} \cup \mathcal{G}^{(l)}(I),
$$

y que $l \in \mathbb{N}^{0}$ es arbitrario, $T_{I}=\bigcup\left\{T^{(j)}(I) \mid 0 \leq j<\omega\right\}$.

Definición 1.3. Sean $l$ de modo que $0 \leq l<\omega$, y sea $a \in T_{I}$. Definimos la función ord $_{I}: T_{I} \mapsto \mathbb{N}^{0}$, de la forma,

$$
\operatorname{ord}_{I}(a)=l \text {, si y solo si, } a \in T^{(l)}(I) \text {. }
$$

Llamaremos a $\operatorname{ord}_{I}(a)$ el orden de a.

Notemos que el orden de $a$ esta bien definido dada la proposición anterior, en especifico el ítem 7.

La siguiente proposición muestra que nuestra definición es una generalización natural del "orden" de [1] página 4.

Proposición 1.4. Sean $a, a_{1}, \ldots, a_{n} \in T_{I}$, y sea $n$ de modo que $1 \leq$ $n<\omega$. Se cumple

1. $a \in I$, entonces ord $_{I}(a)=0$.

2. $a=\left\langle a_{1}, \ldots, a_{n}\right\rangle$, entonces

$$
\operatorname{ord}_{I}(a)=\operatorname{máx}\left\{\operatorname{ord}_{I}\left(a_{1}\right), \ldots, \operatorname{ord}_{I}\left(a_{n}\right)\right\}+1 \text {. }
$$


Demostración. Si $a \in I$, por definición 1.1 ítem $3, a \in T^{(0)}(I)$, y, por definición 1.3, $\operatorname{ord}_{I}(a)=0$. Para $a \in T^{(1)}(I)$, tendríamos, por definición 1.1 ítem $3,\left\{a_{1}, \ldots, a_{n}\right\} \subset T^{(0)}(I)$, así claramente se cumple

$$
\operatorname{ord}_{I}(a)=0+1=\operatorname{máx}\left\{\operatorname{ord}_{I}\left(a_{1}\right), \ldots, \operatorname{ord}_{I}\left(a_{n}\right)\right\}+1 .
$$

Para $a \in T^{(j)}(I)$, con $1<j<\omega$, tenemos por definición 1.1 ítem 2,

$$
\left\{a_{1}, \ldots, a_{n}\right\} \subset \bigcup\left\{\mathcal{G}^{(i)}(I) \mid 0 \leq i<j\right\},
$$

luego por definición 1.3: $\operatorname{ord}_{I}\left(a_{i}\right) \leq j-1$, para $1 \leq i \leq n$. Dada la forma de $a$ sabemos $\left\langle a_{1}, \ldots, a_{n}\right\rangle \notin \mathcal{G}^{(j-1)}(I)$, así

$$
\left\{a_{1}, \ldots, a_{n}\right\} \not \subset \bigcup\left\{\mathcal{G}^{(i)}(I) \mid 0 \leq i<j-1\right\},
$$

en síntesis: existe $a_{q}$, con $1 \leq q \leq n$, que cumple,

$$
a_{q} \notin \bigcup\left\{\mathcal{G}^{(i)}(I) \mid 0 \leq i<j-1\right\}, \mathrm{y}, a_{q} \in \bigcup\left\{\mathcal{G}^{(i)}(I) \mid 0 \leq i<j\right\} .
$$

Luego $a_{q} \in \mathcal{G}^{(j-1)}(I), \mathrm{y}, a_{q} \notin \mathcal{G}^{(j-2)}(I)$, así, $a_{q} \in T^{(j-1)}(I)$, es decir $\operatorname{ord}_{I}\left(a_{q}\right)=j-1$. Como $\operatorname{ord}_{I}\left(a_{i}\right) \leq j-1$, para $1 \leq i \leq n$, entonces, $\operatorname{ord}_{I}\left(a_{q}\right)=\operatorname{máx}\left\{\operatorname{ord}_{I}\left(a_{1}\right), \ldots, \operatorname{ord}_{I}\left(a_{n}\right)\right\}$. Por lo tanto, dado que $\operatorname{ord}_{I}(a)=j$,

$$
\operatorname{ord}_{I}(a)=j=\operatorname{ord}_{I}\left(a_{q}\right)+1=\operatorname{máx}\left\{\operatorname{ord}_{I}\left(a_{1}\right), \ldots, \operatorname{ord}_{I}\left(a_{n}\right)\right\}+1 .
$$

Ahora que disponemos de una buena definición de tipos podemos definir tipos de conjuntos. Luego usaremos esto para definir tipos de estructuras.

Definición 1.5. Sea $O b j$ un conjunto.

1. Diremos que Obj es una colección objetiva de I si cumple:

a) Existe $f$ biyección entre $O b j$ e $I, f: I \mapsto O b j$.

b) $x \in O b j$, entonces $x \neq \emptyset$.

c) para $x, y \in O b j, x \neq y$ si y solo si $x \cap y=\emptyset$. 
2. Para: Obj una colección objetiva de $I, f$ una biyección correspondiente entre $I$ y Obj, definimos $\mathcal{O}_{I}$, la objetivación de $I$, del siguiente modo

a) para $a \in I, \mathcal{O}_{I}(O b j)(a)=f(a) \times\{0\}$.

b) si $a=\left\langle a_{1}, \ldots, a_{n}\right\rangle$, con $a_{1}, \ldots, a_{n} \in T_{I}$, entonces

$\mathcal{O}_{I}(O b j)(a)=\left(\mathbb{P}\left(\mathcal{O}_{I}(O b j)\left(a_{1}\right) \times \ldots \times \mathcal{O}_{I}(O b j)\left(a_{1}\right)\right) \backslash\{\emptyset\}\right) \times\left\{\operatorname{ord}_{I}(a)\right\}$.

c) Sea $l \in \mathbb{N}^{0}$, definimos $\mathcal{P}_{I}^{(l)}$, el peldaño $l$ de $I$, como

$$
\mathcal{P}_{I}^{(l)}(O b j)=\bigcup\left\{\mathcal{O}_{I}(O b j)(a) \mid a \in T^{(l)}(I)\right\} .
$$

3. Definimos $\mathcal{E}_{I}$ la escalera de $I$, de la forma

$$
\mathcal{E}_{I}(O b j)=\bigcup\left\{\mathcal{O}_{I}(O b j)(a) \mid a \in T_{I}\right\} .
$$

4. Definimos $\emptyset^{\omega}$, la relación vacia, de la forma $\emptyset^{\omega}=(\emptyset, \omega)$.

Por cierto en el marco de la definición anterior ítem 1, si Obj cumple 1.a) y 1.b) no necesariamente cumple la propiedad 1.c), en tal caso podemos usar $O b j^{*}=\left\{x^{*} \mid x \in O b j\right\}$, donde $x^{*}=x \times\{x\}$. Notemos que para cada $a^{*}, b^{*}$ distintos entre sí se cumple $a^{*} \cap b^{*}=\emptyset$.

Notemos que $\mathcal{P}_{I}^{(l)}(O b j)$ es un conjunto, puesto que $\left\{\mathcal{O}_{I}(O b j)(a) \mid a \in\right.$ $\left.T^{(l)}(I)\right\}$ es un conjunto. Esto se deduce de la proposición 1.2 ítem 5 y el hecho de que hay una aplicación desde $T^{(l)}(I)$ a $\left\{\mathcal{O}_{I}(O b j)(a) \mid a \in\right.$ $\left.T^{(l)}(I)\right\}$ que es sobreyectiva ${ }^{10}$. Del mismo modo deducimos que $\left\{\mathcal{P}_{I}^{(l)}(O b j) \mid 0 \leq l<\omega\right\}, \mathrm{y}, \mathcal{E}_{I}(O b j)$, son conjuntos.

Con respecto al ítem 3 de la definición anterior notemos que $\mathcal{E}_{I}(O b j)$ es equivalente también

$$
\mathcal{E}_{I}(O b j)=\bigcup\left\{\mathcal{P}_{I}^{(l)} \mid l \in \omega\right\},
$$

y el motivo de definirlo, al modo que fue, es mas que nada dejar en claro que $\mathcal{E}_{I}(O b j)$ es el conjunto de todas las relaciones posibles.

No es difícil notar que un caso particular de lo definido es $I=\{i\}$ y $O b j=\{D\}$ para un $D$ conjunto cualquiera, este caso es importante,

\footnotetext{
${ }^{10}$ Para mas detalle ver página 234, teorema 8.7 del libro [4].
} 
puesto que nos permitirá conectar la definición de estructura que se dará mas adelante con el concepto de estructura en Newton da Costa ${ }^{11}$.

Proposición 1.6. Sean $O b j$ una colección objetiva de $I, a, b \in T_{I}$, $i, j \in \mathbb{N}^{0}$ y $\emptyset^{\omega}$ la relación vacía. Se cumple

1. $\mathcal{O}_{I}(O b j)(a) \neq \emptyset$

2. $\emptyset^{\omega} \notin \mathcal{O}_{I}(O b j)(a)$.

3. $a \neq b$ si y solo si $\mathcal{O}_{I}(O b j)(a) \cap \mathcal{O}_{I}(O b j)(b)=\emptyset$.

4. $a \neq b$ entonces $\mathcal{O}_{I}(O b j)(a) \neq \mathcal{O}_{I}(O b j)(b) .{ }^{12}$

5. $j \neq i$, si y solo si, $\mathcal{P}^{(j)}(O b j) \cap \mathcal{P}^{(i)}(O b j)=\emptyset$.

Demostración. Enumerando las demostraciones:

1. Demostraremos por inducción sobre el orden de $a$. Si $\operatorname{ord}_{I}(a)=0$, entonces $a \in I$. Luego por definición 1.5 ítem 1 y 2

$$
\mathcal{O}_{I}(O b j)(a)=f(a) \times\{0\} \neq \emptyset .
$$

Ahora, para $\operatorname{ord}_{I}(a)=m$, tenemos

$$
\mathcal{O}_{I}(O b j)(a)=\left(\mathbb{P}\left(\mathcal{O}_{I}(O b j)\left(a_{1}\right) \times \ldots \times \mathcal{O}_{I}(O b j)\left(a_{n}\right)\right) \backslash\{\emptyset\}\right) \times\left\{\operatorname{ord}_{I}(a)\right\},
$$

donde $a=\left\langle a_{1}, \ldots, a_{n}\right\rangle$. Notemos que por proposición 1.4 ítem 2 $\operatorname{ord}_{I}\left(a_{i}\right)<m$, para $1 \leq i \leq n$, así, por hipótesis de inducción, tenemos que $\mathcal{O}_{I}(O b j)\left(a_{i}\right) \neq \emptyset$. Luego

$$
\mathbb{P}\left(\mathcal{O}_{I}(O b j)\left(a_{1}\right) \times \ldots \times \mathcal{O}_{I}(O b j)\left(a_{n}\right)\right) \backslash\{\emptyset\} \neq \emptyset .
$$

Por lo tanto, en virtud de la igualdades mostradas $\mathcal{O}_{I}(O b j)(a) \neq$ $\emptyset$.

2. Claramente se cumple este ítem, puesto que $\omega \notin \mathbb{N}^{0}$.

\footnotetext{
${ }^{11}$ Ver trabajo [1] página 4.

${ }^{12}$ Por cierto diremos que $\mathcal{O}_{I}(O b j)$ es inyectiva a pesar de no se una función, el motivo de esto, es que la propiedad mostrada tiene la cualidad de ser.
}

Manuscrito - Rev. Int. Fil., Campinas, v. 38, n.2, pp.89-154, jul.-ago. 2015. 
3. De derecha a izquierda. La demostración sera por inducción sobre el orden. Sea $a \neq b, a, b \in I$, luego tenemos que

$$
\mathcal{O}_{I}(O b j)(a) \cap \mathcal{O}_{I}(O b j)(b)=\emptyset,
$$

por definición 1.5 ítem 1 y 2. Ahora sean $\operatorname{ord}_{I}(a)=m_{1}, \mathrm{y}$, $\operatorname{ord}_{I}(b)=m_{2}$, claramente si $m_{1} \neq m_{2}$, entonces,

$$
\mathcal{O}_{I}(O b j)(a) \cap \mathcal{O}_{I}(O b j)(b)=\emptyset,
$$

dada la definición 1.5 ítem 2. Si $m_{1}=m_{2}$, tenemos que $a, b \in$ $T^{\left(m_{1}\right)}(I)$, en tal caso, $a=\left\langle a_{1}, \ldots, a_{n}\right\rangle, \mathrm{y}, b=\left\langle b_{1}, \ldots, b_{n^{\prime}}\right\rangle$. Sin perdida de generalidad supongamos $n \leq n^{\prime}$, luego debe ocurrir:

a) $\mathrm{O}$ bien existe $1 \leq q \leq n$, de modo que $a_{q} \neq b_{q}$.

b) O bien no existe $1 \leq q \leq n$, de modo que $a_{q} \neq b_{q}$, pero $n<n^{\prime}$.

En el primer caso por hipótesis de inducción $\mathcal{O}_{I}(O b j)\left(a_{q}\right) \cap$ $\mathcal{O}_{I}(O b j)\left(b_{q}\right)=\emptyset$, luego

$\mathcal{O}_{I}(O b j)\left(a_{1}\right) \times \ldots \times \mathcal{O}_{I}(O b j)\left(a_{n}\right) \cap \mathcal{O}_{I}(O b j)\left(b_{1}\right) \times \ldots \times \mathcal{O}_{I}(O b j)\left(b_{n^{\prime}}\right)=\emptyset$,

así la intersección de $\mathbb{P}\left(\mathcal{O}_{I}(O b j)\left(a_{1}\right) \times \ldots \times \mathcal{O}_{I}(O b j)\left(a_{n}\right)\right)$ con $\left.\mathbb{P}\left(\mathcal{O}_{I}(O b j)\left(b_{1}\right) \times \ldots \times \mathcal{O}_{I}(O b j)\left(b_{n^{\prime}}\right)\right)\right)=\{\emptyset\}$ es vaciá, de lo cual deducimos, por definición 1.5 ítem $2, \mathcal{O}_{I}(O b j)(a) \cap \mathcal{O}_{I}(O b j)(b)=$ $\emptyset$. Para el caso $b)$, es obvio que la intersección de $\mathcal{O}_{I}(O b j)\left(a_{1}\right) \times$ $\ldots \times \mathcal{O}_{I}(O b j)\left(a_{n}\right) \operatorname{con} \mathcal{O}_{I}(O b j)\left(b_{1}\right) \times \ldots \times \mathcal{O}_{I}(O b j)\left(b_{n^{\prime}}\right)$, es vaciá. Del mismo modo que el punto anterior deducimos

$$
\mathcal{O}_{I}(O b j)(a) \cap \mathcal{O}_{I}(O b j)(b)=\emptyset .
$$

De derecha a izquierda, supongamos que $a=b$, luego

$$
\mathcal{O}_{I}(O b j)(a) \cap \mathcal{O}_{I}(O b j)(b)=\mathcal{O}_{I}(O b j)(a),
$$

pero por el ítem 1 de esta proposición $\mathcal{O}_{I}(\operatorname{Obj})(a) \neq \emptyset$, lo cual produce un claro contrasentido con la hipótesis, por lo tanto $a \neq$ $b$.

4. Por el ítem 1 y 3 tenemos $a \neq b$ entonces $\mathcal{O}_{I}(O b j)(a) \neq \mathcal{O}_{I}(O b j)(b)$. Luego $\mathcal{O}_{I}(O b j)$ es inyectiva. 
5. Si $x \in \mathcal{P}_{I}^{(j)}(O b j)$, por definición 1.5 ítem 2, entonces $x=(R, j)$, con $x \in \mathcal{O}_{I}(O b j)(a)$, para algún $a \in T^{(j)}(I)$. Del mismo modo obtenemos que $y=\left(R^{\prime}, i\right)$, con $y \in \mathcal{O}_{I}(O b j)(b)$, para algún $b \in$ $T^{(i)}(I)$. Claramente si $j \neq i$ entonces $\mathcal{P}_{I}^{(j)}(O b j) \cap \mathcal{P}_{I}^{(i)}(O b j)=\emptyset$, dada la construcciones de lo elementos da cada conjunto.

De derecha a izquierda. Si $\mathcal{P}^{(j)}(O b j) \cap \mathcal{P}^{(j)}(O b j)=\emptyset$, entones por la proposición 1.2 ítem 7 , y las construcciones mostradas, tenemos $i \neq j$, o bien, $\mathcal{P}_{I}^{(j)}(O b j)=\emptyset$. Como $\mathcal{P}_{I}^{(j)}(O b j)=\emptyset$ es un contrasentido con el ítem 1, puesto que, dada la proposición 1.2 ítem 6 , existe $a \in T^{(j)}(I)$, de modo que

$$
\emptyset \neq \mathcal{O}_{I}(O b j)(a) \subset \mathcal{P}_{I}^{(j)}(O b j) .
$$

Por lo tanto $j \neq i$.

En lo que se viene una definición del tipo de una relación, formalizando lo expuesto en el trabajo [1] pagina 5.También presentamos un operador que usaremos en la definición 1.9 ítem 4.

Definición 1.7. Sea Obj una colección objetiva de $I, a \in T_{I}, R \in$ $\mathcal{E}_{I}(O b j)$.

1. $R$ es de tipo a en $O b j$, si y solo si, $R \in \mathcal{O}_{I}(O b j)(a)$.

2. Si $a \in I$, diremos que $R$ es un a-individuo en $O b j$

3. El operador $\mathcal{R e l}\left(\mathcal{O}_{I}\right)$, la relación en $\mathcal{O}_{I}$, es de la forma

$$
\operatorname{Rel}\left(\mathcal{O}_{I}\right)(R)=\left\{\begin{array}{lll}
X & \text { para } & R=\left(X, \operatorname{ord}_{I}(a)\right) \in \mathcal{O}_{I}(O b j)(a) \\
\emptyset & \text { para } & R=\emptyset^{\omega}
\end{array}\right.
$$

Notemos que el ítem 1 de esta definición es acertado dada la proposición 1.6 ítem 3, es decir, cada relator tiene un solo tipo asociado.

El motivo para definir el concepto de tipo de una relación de una manera algo distinta de lo hecho en [1] es eliminar algunas ambigüedades. Tomemos $\emptyset$, el conjunto vacío, que en términos del trabajo [1] cumple $\emptyset \in t(\langle i\rangle)$ y $\emptyset \in t(\langle\langle i\rangle\rangle)$, luego tenemos, en términos de lo expuesto en la pagina 4 y 5 de [1], que el vacío es de tipo $\langle i\rangle$ y $\langle\langle i\rangle\rangle$, 
además, es de orden 1 y 2 al mismo tiempo. El camino que se ha tomado para solucionar esto es considerar que la relación vacía es una relación especial que no tiene tipo.

Notemos que si $I=\{i\}$ recuperamos la definición del trabajo [1] puesto que $T=T_{I}$. Aunque con un cierto reparo, puesto que las relaciones en este mundo son un par: un conjunto relación y su orden.

Ejemplo 1.8. Veamos:

1. Sea Obj $=\left\{\mathbb{N}^{0}\right\}$, sea $I=\{i\}$, donde es claro que Obj es una colección objetiva ${ }^{13}$ de I, y en tal caso:

a) Si suc $=\left\{(n, m) \in \mathbb{N}^{0} \times \mathbb{N}^{0} \mid n=m+1\right\}$, entonces definimos $s u c^{1}=(\{((n, 0),(m, 0)) \mid(n, m) \in s u c\}, 1) \in \mathcal{O}_{I}(\operatorname{Obj})(\langle i, i\rangle)$, luego suc ${ }^{1}$ es de tipo $\langle i, i\rangle$.

b) $S i+=\left\{\left(n_{1}, n_{2}, n_{3}\right) \in \mathbb{N}^{0} \times \mathbb{N}^{0} \times \mathbb{N}^{0} \mid n_{1}+n_{2}=n_{3}\right\}$, entonces $+{ }^{1}=\left(\left\{\left(\left(n_{1}, 0\right),\left(n_{2}, 0\right),\left(n_{3}, 0\right)\right) \mid\left(n_{1}, n_{2}, n_{3}\right) \in+\right\}, 1\right)$, $y+{ }^{1} \in \mathcal{O}_{I}(O b j)(\langle i, i, i\rangle)$, luego $+{ }^{1}$ es de tipo $\langle i, i, i\rangle$.

c) $S i \bullet=\left\{\left(n_{1}, n_{2}, n_{3}\right) \in \mathbb{N}^{0} \times \mathbb{N}^{0} \times \mathbb{N}^{0} \mid n_{1} \bullet n_{2}=n_{3}\right\}$, entonces $\bullet^{1}=\left(\left\{\left(\left(n_{1}, 0\right),\left(n_{2}, 0\right),\left(n_{3}, 0\right)\right) \mid\left(n_{1}, n_{2}, n_{3}\right) \in+\right\}, 1\right)$, $y \bullet^{1} \in \mathcal{O}_{I}(O b j)(\langle i, i, i\rangle)$, luego $\bullet^{1}$ es de tipo $\langle i, i, i\rangle$.

2. Sea $(G, \star)$ un grupo, sea $O b j=\{G\}$, sea $I=\{i\}$, donde es claro que Obj es una colección objetiva ${ }^{14}$ de I. En tal caso:

a) $S i \star=\left\{\left(g_{1}, g_{2}, g_{3}\right) \in G \times G \times G \mid q_{1} \star g_{2}=g_{4}\right\}$, entonces

$$
\begin{gathered}
\star^{1}=\left(\left\{\left(\left(n_{1}, 0\right),\left(n_{2}, 0\right),\left(n_{3}, 0\right)\right) \mid\left(n_{1}, n_{2}, n_{3}\right) \in \bullet\right\}, 1\right), \\
y \star^{1} \in \mathcal{O}_{I}(O b j)(\langle i, i, i\rangle), \text { luego } \star^{1} \text { es de tipo }\langle i, i, i\rangle .
\end{gathered}
$$

b) Si $e_{G}$ es el neutro, entonces $e_{G}^{1}=\left(\left\{e_{G}\right\}, 1\right)$ es de tipo $\langle i\rangle$.

\footnotetext{
${ }^{13}$ Satisface las condiciones 1.a), 1.b) y 1.c) de la definición 1.5.

${ }^{14}$ Satisface las condiciones 1.a), 1.b) y 1.c) de la definición 1.5.
} 
3. Sea $\left(D, \mathfrak{T}_{D}\right)$ un espacio topológico, supongamos que $D \cap \mathbb{P}(D)=$ $\emptyset$. Notemos que $O b j=\{D, \mathbb{P}(D)\}$ es una colección objetiva de $I=\{i, j\}$, donde es claro que Obj es una colección objetiva ${ }^{15}$ de I. En tal caso:

a) Sea $\in_{D}=\{(a, b) \in D \times \mathbb{P}(D) \mid a \in b\}$, entonces

$$
\in_{D}^{1}=\left(\left\{\left((a, 0),(b, 0) \mid(a, b) \in \in_{D}\right\}, 1\right) \in \mathcal{O}_{I}(O b j)(\langle i, j\rangle),\right.
$$

asi $\in_{D}^{1}$ es de tipo $\langle i, j\rangle$.

b) Definimos

$$
\mathfrak{T}_{D}^{1}=(\{(a, 0) \mid a \in \mathfrak{T}\}, 1) \in \mathcal{O}_{I}(O b j)(\langle j\rangle),
$$

luego $\mathfrak{T}_{D}^{1}$ es de tipo $\langle j\rangle$.

4. Sea $\left(D, \mathfrak{U}_{D}\right)$, una variedad topológica de dimensión $n$, con $\mathfrak{U}_{D}$ un atlas maximal. Suponiendo que Obj $=\left\{D, \mathbb{P}(D), \mathbb{R}^{n}, \mathbb{P}\left(\mathbb{R}^{n}\right)\right\}$ es una colección objetiva de $I=\{i, j, o, p\}$. Entonces, para $\varphi \in \mathfrak{U}_{D}$, $\varphi \subset A \times B$, con $A \subset D$ y $B \subset \mathbb{R}^{n}$, además de otras propiedades esenciales, pero con lo dicho es suficiente para definir

$$
\varphi^{1}=(\{((a, 0),(b, 0)) \mid(a, b) \in \varphi\}, 1) \subset \mathcal{O}_{I}(O b j)(\langle i, o\rangle),
$$

luego $\varphi^{1}$ es de tipo $\langle i, o\rangle$. Por cierto para $O b j, I$ definido más arriba, tenemos que $\mathfrak{T}_{D}^{1}$ es de tipo $\langle j\rangle, \mathfrak{T}_{\mathbb{R}^{n}}^{1}$ es de tipo $\langle p\rangle, \in_{D}^{1}$ es de tipo $\langle i, o\rangle, y, \in_{\mathbb{R}^{n}}^{1}$ es de tipo $\langle o, p\rangle$.

Definición 1.9. Sea Obj una colección objetiva de I.

1. Una pre-estructura en Obj, es una función $\Psi: \mathbb{N}^{0} \cup\{\omega\} \mapsto$ $\mathcal{E}_{I}(O b j) \cup\left\{\emptyset^{\omega}\right\}$, que cumple

$$
\Psi(l) \subset \mathcal{P}_{I}^{(l)}(O b j), \text { para } l \in \mathbb{N}^{0}, y, \Psi(\omega)=\emptyset^{\omega} .
$$

2. Sea $a \in T_{I}$, definimos el universo de tipo a de $\Psi$ como $U^{a}(\Psi)=\Psi\left(\operatorname{ord}_{I}(a)\right) \cap \mathcal{O}_{I}(O b j)(a)$.

3. El universo de $\Psi, U(\Psi)$, como $U(\Psi)=\bigcup \Psi\left[\mathbb{N}^{0}\right]$, donde $\Psi[]$, es el operador imagen de $\Psi$.

\footnotetext{
${ }^{15}$ Satisface las condiciones 1.a), 1.b) y 1.c) de la definición 1.5.
} 
4. $\Psi$ es una estructura en $O b j$, si y solo si: es una pre-estructura, y para cada $R \in U(\Psi)$ de tipo $a=\left\langle a_{1}, \ldots, a_{n}\right\rangle$ en $T_{I}$

$$
\operatorname{Rel}\left(\mathcal{O}_{I}\right)(R) \subset U^{a_{1}}(\Psi) \times \ldots \times U^{a_{n}}(\Psi) .
$$

Por lo general denotaremos las estructuras como sucesiones en $\mathbb{N}^{0}$, puesto que su ultimo valor es el mismo para cualquiera y es irrelevante para la diferenciación entre ellas.

Puede que la definición parezca poco intuitiva y difiera de lo mostrado por Newton da Costa en [1], sin embargo ésta es capaz de modelar bastante bien las estructuras que él plantea. Para ello tomemos la estructura $e=\left\langle D, r_{l}\right\rangle$, en el sentido de [1], lo primero que haremos es nombrar $R_{l}$ al conjunto de los elementos de $r_{l}$. Luego, fijando $I=\{i\}$ y $O b j=\{D\}$, transformamos las relaciones de $R_{l}$ como hemos mencionado en el párrafo antecedente al ejemplo 1.8. El conjunto de los elementos transformados lo denotaremos por $R_{l}^{*}$. Ya estamos casi listos, para terminar construimos la sucesión

$$
\left\langle D \times\{0\}, R_{l}^{*} \cap \mathcal{P}_{I}^{(1)}(O b j), R_{l}^{*} \cap \mathcal{P}_{I}^{(2)}(O b j), \ldots, R_{l}^{*} \cap \mathcal{P}_{I}^{(n)}(O b j), \ldots\right\rangle,
$$

que es el análogo de la estructura de Newton da Costa $^{16}$ en esta construcción.

Puede resultar curioso el hecho que se definiera pre-estructura y no directamente estructura. El motivo de ello es que la pre-estructura posee algunos casos anómalos. Por ejemplo la siguiente pre-estructura: $\left\langle\mathbb{R}^{0},\left\{+_{\mathbb{C}}^{1}, \cdot_{\mathbb{C}}^{1}\right\}, \emptyset, \ldots\right\rangle$, donde $\mathbb{R}^{0}=\mathbb{R} \times\{0\}, \mathrm{y}+{ }_{\mathbb{C}}^{1}, \cdot{ }_{\mathbb{C}}^{1}$ son la suma y el productos en los complejos modificados para estar acorde a la definición anterior.

Definición 1.10. Sea $\Psi$ una pre-estructura en $O b j$, con $O b j$ una colección objetiva de I. Definimos:

1. Para $R \in U(\Psi)$ :

$$
\operatorname{ord}_{I}(R)=\operatorname{ord}_{I}(a) \text {, si } R \in \mathcal{O}_{I}(O b j)(a) \text { para algún } a \in T_{I} .
$$

Llamaremos a $\operatorname{ord}_{I}(R)$, el orden de $R$.

\footnotetext{
${ }^{16}$ Ver trabajo [1] página 4.
} 
2. El orden de $\Psi$ es de la forma:

$$
\operatorname{ord}_{I}(\Psi)=\left\{\begin{array}{cl}
\operatorname{máx}\left\{\operatorname{ord}_{I}(R) \mid R \in U(\Psi)\right\} & , \text { si este max existe } \\
\omega & , \text { en otro caso. }
\end{array}\right.
$$

Notemos que esta definición está bien fundada dada la proposición 1.6 ítem 2 y 3 . Esto nos asegura que ningún relator tendrá mas de un tipo; del mismo modo, el orden asociado es único.

\section{Ejemplos de estructuras}

\section{Estructura simple y múltiple}

Hemos visto en varios ejemplos que el caso particular de estructuras en el cual se fija $I=\{i\}$ y $O b j=\{D\}$, para $D$ un conjunto cualquiera, es muy recurrente al momento de tratar de entender a Newton da Costa, tal hecho es el que motiva la siguiente definición.

Definición 2.1. Sea Obj una colección objetiva de I. Diremos que

1. Si Obj $=\{D\}$, con $D$ un conjunto cualquiera, una estructura en Obj será denotada por e, y será llamada estructura simple.

2. Si \#Obj $>1$, donde \# es el operador cardinal, una estructura en Obj será denotada por $\mu$, y será llamada estructura múltiple.

Las estructuras simples son nuestro análogo a Newton da Costa. Las estructuras múltiples nos permitirá, hablar de estructuras de varios individuos. Sin embargo, según Newton da Costa, son prescindibles, puesto que se pueden reducir a una estructura simple, pero como no se ha demostrado tal afirmación trabajaré con ellas. En mi opinión las estructuras múltiples simplifican la traducción de conceptos matemáticos a estructuras, como vimos en el ejemplo 1.8.

Ejemplo 2.2. Usando el ejemplo 1.8 veamos

1. Sea $O b j=\left\{\mathbb{N}^{0}\right\}$, definimos e como:

a) $e(0)=\mathbb{N}^{0} \times\{0\}$.

b) $e(1)=\left\{+^{1}, \bullet^{1}, s u c^{1}\right\}$. 
c) $e(l)=\emptyset$, para $1<l<\omega$.

Esta estructura permite de modelar la aritmética. Generalmente se denota por

$$
e=\left\langle\mathbb{N}^{0} \times\{0\},\left\{+^{1}, \bullet^{1}, s u c^{1}\right\}\right\rangle .
$$

2. Sea $O b j=\{G\}$, definimos e como:

a) $e(0)=G \times\{0\}$.

b) $e(1)=\left\{\star^{1}, e_{G}^{1}\right\}$.

c) $e(l)=\emptyset$, para $1<l<\omega$.

Esta estructura permite de modelar la noción de grupo. Generalmente se denota por

$$
e=\left\langle G \times\{0\},\left\{\star^{1}, e_{G}^{1}\right\}\right\rangle .
$$

3. Sea $O b j=\{D, \mathbb{P}(D)\}$, definimos $\mu$ como:
a) $\mu(0)=(D \cup \mathbb{P}(D)) \times\{0\}$.
b) $\mu(1)=\left\{\mathfrak{T}_{D}^{1}, \in_{D}^{1}\right\}$.
c) $\mu(l)=\emptyset$, para $1<l<\omega$.

Esta estructura permite de modelar la noción de topología. Generalmente se denota por

$$
\mu=\left\langle(D \cup \mathbb{P}(D)) \times\{0\},\left\{\mathfrak{T}_{D}^{1}, \in_{D}^{1}\right\}\right\rangle .
$$

4. Sea Obj $=\left\{D, \mathbb{P}(D), \mathbb{R}^{n}, \mathbb{P}\left(\mathbb{R}^{n}\right)\right\}$, definimos $\mu$ como:

a) $\mu(0)=\left(D \cup \mathbb{P}(D) \cup \mathbb{R}^{n} \cup \mathbb{P}\left(\mathbb{R}^{n}\right)\right) \times\{0\}$.

b) $\mu(1)=\left\{\mathfrak{T}_{D}^{1}, \in_{D}^{1}, \mathfrak{T}_{\mathbb{R}^{n}}^{1}, \in_{\mathbb{R}^{n}}^{1}\right\} \cup \mathfrak{U}_{D}^{*}$, donde $\mathfrak{U}_{D}^{*}=\left\{\varphi^{1} \mid \varphi \in\right.$ $\left.\mathfrak{U}_{D}\right\}$

c) $\mu(l)=\emptyset$, para $1<l<\omega$.

Esta estructura permite de modelar la noción de variedad topológica. Generalmente se denota por

$$
\mu=\left\langle\left(D \cup \mathbb{P}(D) \cup \mathbb{R}^{n} \cup \mathbb{P}\left(\mathbb{R}^{n}\right)\right) \times\{0\},\left\{\mathfrak{T}_{D}^{1}, \in_{D}^{1}, \mathfrak{T}_{\mathbb{R}^{n}}^{1}, \in_{\mathbb{R}^{n}}^{1}\right\} \cup \mathfrak{U}_{D}^{*}\right\rangle .
$$

En lo que sigue si $I=\{i\}$, entonces $T_{I}$ será denotado por $T$, ord $d_{I}$ por ord, $\mathcal{O}_{I}$ por $\mathcal{O}, \mathcal{E}_{I}$ por $\mathcal{E}, \mathcal{P}_{I}^{(l)}$ por $\mathcal{P}^{(l)}$, como en el articulo [1]. 


\section{Estructura análoga}

A continuación construiremos las herramientas necesarias para poder reducir estructuras múltiples a estructuras simples. Para ello construiremos las transformaciones de tipos convenientes. Después construiremos colecciones objetivas convenientes para estas transformaciones, y por último construiremos la estructura que permitirá la reducción.

Definición 2.3. Sea $J$ un conjunto arbitrario donde $T_{J}$ es un conjunto de tipos y sea $I=\{i\}$ como arriba. Definimos, la función singular de $J$, como una función $\mathcal{S}_{J}: T_{J} \mapsto T$, que cumple

1. $\mathcal{S}_{J}(a)=i$, para todo $a \in J$.

2. si $a=\left\langle a_{1}, \ldots, a_{n}\right\rangle$, entonces $\mathcal{S}_{J}(a)=\left\langle\mathcal{S}_{J}\left(a_{1}\right), \ldots, \mathcal{S}_{J}\left(a_{n}\right)\right\rangle$.

Proposición 2.4. Sea \# es el operador cardinal. Se cumple que:

1. para todo $a \in T_{J}, \operatorname{ord}_{J}(a)=\operatorname{ord}\left(\mathcal{S}_{J}(a)\right)$.

2. $\mathcal{S}_{J}$ es sobreyectiva.

3. Sea $a \in T$, se cumple que:

$$
\# \mathcal{S}_{J}[\{a\}] \leq \omega \text {, si y solo si, } \# J \leq \omega .
$$

Demostración. Enumerando las demostraciones:

1. La demostración será por inducción sobre el orden de $a \in T_{J}$, sea $\operatorname{ord}_{J}(a)=0$, luego necesariamente $a \in J$, de lo contrario por proposición 1.4 ítem $2 \operatorname{ord}_{I}(a) \geq 1$. Así $a \in J$ y $\mathcal{S}_{J}(a)=i$, luego por definición $1.3 \operatorname{ord}\left(\mathcal{S}_{J}(a)\right)=0$. Por lo tanto $\operatorname{ord}_{J}(a)=$ $\operatorname{ord}\left(\mathcal{S}_{I}(a)\right)$.

Para $\operatorname{ord}_{J}(a) \geq 1$, tenemos que $a=\left\langle a_{1}, \ldots, a_{n}\right\rangle$, de lo contrario $a \in J$ lo cual es una clara contradicción con la proposición 1.4 ítem 1. Luego:

$$
\operatorname{ord}_{J}(a)=\operatorname{máx}\left\{\operatorname{ord}_{J}\left(a_{1}\right), \ldots, \operatorname{ord}_{J}\left(a_{n}\right)\right\}+1,
$$

$\operatorname{como}_{\operatorname{ord}}\left(a_{j}\right)<\operatorname{ord}_{J}(a)$, para $1 \leq j \leq n$, por hipótesis de inducción

$$
\operatorname{ord}_{J}(a)=\operatorname{máx}\left\{\operatorname{ord}\left(\mathcal{S}_{J}\left(a_{1}\right)\right), \ldots, \operatorname{ord}\left(\mathcal{S}_{J}\left(a_{n}\right)\right)\right\}+1,
$$

de lo cual se deduce, por proposición 1.4, $\operatorname{ord}_{J}(a)=\operatorname{ord}\left(\mathcal{S}_{J}(a)\right)$. 
2. Demostraremos por inducción sobre el orden. Sea $a \in T$ de modo que $\operatorname{ord}(a)=0$, luego necesariamente $a=i$, de lo contrario tenemos un contrasentido con la proposición 1.4. Ahora escojamos cualquier $j \in J$ para obtener por el ítem 1 y definición 2.3

$$
\operatorname{ord}_{J}(j)=\operatorname{ord}\left(\mathcal{S}_{J}(j)\right)=\operatorname{ord}(i)=0,
$$

lo cual afirma que $\mathcal{S}_{J}(j)=i$.

Para $\operatorname{ord}(a) \geq 1$, tenemos que $a=\left\langle a_{1}, \ldots, a_{n}\right\rangle$, para $1 \leq n<\omega$, luego, por proposición 1.4

$$
\operatorname{ord}(a)=\operatorname{máx}\left\{\operatorname{ord}\left(a_{1}\right), \ldots, \operatorname{ord}\left(a_{n}\right)\right\}+1,
$$

usando hipótesis de inducción, ya que $\operatorname{ord}\left(a_{l}\right)<\operatorname{ord}(a)$ para $1 \leq l \leq n$, existe $b_{l} \in T_{J}$, tal que $\mathcal{S}_{J}\left(b_{l}\right)=a_{l}$. Así por definición 2.3

$$
a=\left\langle\mathcal{S}_{J}\left(a_{1}\right), \ldots, \mathcal{S}_{J}\left(a_{n}\right)\right\rangle=\mathcal{S}_{J}\left(\left\langle b_{1}, \ldots, b_{n}\right\rangle\right) .
$$

Por lo tanto $\mathcal{S}_{I}$ es sobreyectiva.

3. Sea $\mathcal{S}_{J}^{-1}$ es la imagen inversa del conjunto bajo $\mathcal{S}_{J}$. La demostración sera por inducción sobre el $\operatorname{ord}(a)$.

- Para $\operatorname{ord}(a)=0$ tenemos que $a=i$ y $\mathcal{S}_{J}[\{i\}]=J$, así claramente $\# \mathcal{S}_{J}^{-1}[\{i\}] \leq \omega$, si y solo si $\# J \leq \omega$.

- Si $\operatorname{ord}(a)>0$, luego $a=\left\langle a_{1}, \ldots, a_{n}\right\rangle$, para algún $n \in$ $\mathbb{N}$. Ahora veamos que si $a^{\prime} \in \mathcal{S}_{J}^{-1}[\{a\}]$, entonces $a^{\prime}=$ $\left\langle a_{1}^{\prime}, \ldots, a_{m}^{\prime}\right\rangle$, para algún $m \in \mathbb{N}$, esto viene dado por el ítem 1 de esta proposición y el contrasentido que se produce con la proposición 1.4 si $m=0$. Mas aún, podemos deducir que $m=n$ y $\mathcal{S}_{J}\left(a_{j}\right)=a_{j}^{\prime}$, para $1 \leq j \leq n$. Lo que equivale $a_{j}^{\prime} \in \mathcal{S}_{J}^{-1}\left[\left\{a_{j}\right\}\right]$, para $1 \leq j \leq n$. Como $a^{\prime}$ es arbitrario es arbitrario tenemos que

$$
\# \mathcal{S}_{J}^{-1}[\{a\}]=\#\left(\mathcal{S}_{J}^{-1}\left[\left\{a_{1}\right\}\right] \times \ldots \times \mathcal{S}_{J}^{-1}\left[\left\{a_{n}\right\}\right]\right) .
$$

Por hipótesis de inducción, ya que por proposición 1.4, $\operatorname{ord}\left(a_{j}\right)<\operatorname{ord}(a)$ para $1 \leq j \leq n$, tenemos que: $\# \mathcal{S}_{J}^{-1}\left[\left\{a_{j}\right\}\right] \leq \omega$, si y solo si $\# J \leq \omega$. Como $\mathcal{S}_{J}^{-1}[\{a\}] \leq \omega$, es equivalente a $\# \mathcal{S}_{J}^{-1}\left[\left\{a_{j}\right\}\right] \leq \omega$, para $1 \leq j \leq n$. Por lo tanto $\# \mathcal{S}_{J}^{-1}[\{a\}] \leq \omega$, equivale $\# J \leq \omega$. 
Para lo que se viene, sea $O b j$ una colección objetiva para $I$ arbitrario. Entonces, notemos que $O b j_{i}=\{\bigcup O b j\}$ es una colección objetiva de $\{i\}$. Usamos $O b j_{i}$ para crear una estructura conveniente para la reducción de individuos.

Proposición 2.5. Sean: Obj una colección objetiva para $I, O_{b j}$ como arriba, $l \in \mathbb{N}^{0}$, y $a \in T_{I}$.

1. $\bigcup\left\{\mathcal{O}_{I}(O b j)(a) \mid \in I\right\}=\mathcal{O}\left(O b j_{i}\right)(i)$, es decir $\mathcal{P}_{I}^{(0)}(O b j)=\mathcal{P}^{(0)}\left(O b j_{i}\right)$.

2. $\mathcal{O}_{I}(O b j)(a) \subset \mathcal{O}\left(O b j_{i}\right)\left(\mathcal{S}_{I}(a)\right)$.

3. $R \subset \mathcal{O}_{I}(O b j)(a), y, R \neq \emptyset$, entonces $\left(R, \operatorname{ord}_{I}(a)+1\right)$ es de tipo $\left\langle\mathcal{S}_{I}(a)\right\rangle$ en $\operatorname{Obj}_{i}$.

4. si $R$ es de tipo a en $O b j$, entonces $R$ es de tipo $\mathcal{S}_{I}(a)$ en $O b j_{i}$.

5. si $R$ es un a-individuo en $O b j$ entonces $R$ es un individuo en $O b j_{i}$.

6. $\mathcal{P}_{I}^{(l)}(O b j) \subset \mathcal{P}^{(l)}\left(O b j_{i}\right)$.

7. $\mathcal{E}_{I}(O b j) \subset \mathcal{E}\left(O b j_{i}\right)$.

8. $R \in \mathcal{P}_{I}^{(l)}(O b j)$, para algún $l \in \mathbb{N}$, entonces $\operatorname{Rel}\left(\mathcal{O}_{I}\right)(R)=\operatorname{Rel}(\mathcal{O})(R)$.

Demostración. Enumerando las demostraciones:

1. Veamos que, por definición 1.5 ítem 2,

$$
\bigcup\left\{\mathcal{O}_{I}(O b j)(a) \mid a \in I\right\}=(\bigcup\{f(a) \mid a \in I\}) \times\{0\}=\mathcal{O}\left(O b j_{i}\right)(i),
$$

donde $f$ es la biyección de $I$ en $O b j$. Claramente por definición 1.5 ítem 2 y lo mencionado,

$$
\mathcal{P}_{I}^{(0)}(O b j)=\mathcal{P}^{(0)}\left(O b j_{i}\right)
$$


2. La demostración sera por inducción sobre el orden de $a \in T_{I}$. Para $\operatorname{ord}_{I}(a)=0$, entonces $a \in I$, luego por el ítem 1 de esta proposición y la definición 2.3,

$$
\mathcal{O}_{I}(O b j)(a) \subset \mathcal{O}(O b j)(i)\left(\mathcal{S}_{I}(a)\right) .
$$

Para $\operatorname{ord}_{I}(a) \geq 1$, tenemos $a=\left\langle a_{1}, \ldots, a_{n}\right\rangle$. Usando la proposición 1.4, tenemos $\operatorname{ord}_{I}\left(a_{j}\right)<\operatorname{ord}_{I}(a)$, para $1 \leq j \leq n$, luego por hipótesis de inducción, la proposición 2.4 ítem 1 y definición 1.5 item 2 ,

$\mathcal{O}_{I}(O b j)(a) \subset\left(\mathbb{P}\left(\mathcal{O}\left(O b j_{i}\right)\left(a_{1}\right) \times \ldots \times \mathcal{O}\left(O b j_{i}\right)(a)\right)\right) \times\left\{\operatorname{ord}\left(\mathcal{S}_{I}(a)\right)\right\}$.

Por lo tanto, por definición 1.5 ítem 2,

$$
\mathcal{O}_{I}(O b j)(a) \subset \mathcal{O}\left(O b j_{i}\right)\left(\mathcal{S}_{I}(a)\right) .
$$

3. Notemos que por hipótesis e ítem anterior tenemos, para todo $a \in T_{I}$,

$$
R \in \mathbb{P}\left(\mathcal{O}_{I}(O b j)(a)\right) \backslash\{\emptyset\} \subset \mathbb{P}\left(\mathcal{O}\left(O b j_{i}\right)\left(\mathcal{S}_{I}(a)\right)\right) \backslash\{\emptyset\},
$$

luego tenemos que

$$
\left(R, \operatorname{ord}_{I}(a)+1\right) \in\left(\mathbb{P}\left(\mathcal{O}\left(O b j_{i}\right)\left(\mathcal{S}_{I}(a)\right)\right) \backslash\{\emptyset\}\right) \times\left\{\operatorname{ord}_{I}(a)+1\right\} .
$$

Pensando en la pertenencia mostrada, veamos que, por proposición 2.4 ítem 1 ,

$$
\operatorname{ord}_{I}(\langle a\rangle)=\operatorname{ord}_{I}(a)+1=\operatorname{ord}_{I}\left(\mathcal{S}_{I}(a)\right)+1=\operatorname{ord}\left(\mathcal{S}_{I}(a)\right) .
$$

Luego, por definición 1.5 ítem 2,

$$
\left(R, \operatorname{ord}_{I}(a)+1\right) \in \mathcal{O}\left(O b j_{i}\right)\left(\mathcal{S}_{I}(a)\right) .
$$

Por lo tanto $\left(R, \operatorname{ord}_{I}(a)+1\right)$ es de tipo $\mathcal{S}_{I}(a)$ en $\operatorname{Obj}_{i}$.

4. Consecuencia directa del ítem 2, puesto que: $R$ de tipo $a$ en $O b j$, entonces $R \in \mathcal{O}_{I}(O b j)(a)$, luego $R \in \mathcal{O}\left(O b j_{i}\right)\left(\mathcal{S}_{I}(a)\right)$, así $R$ es de tipo $\mathcal{S}_{I}(a)$ en $O b j_{i}$. 
5. Consecuencia directa del ítem anterior.

6. Demostraremos por inducción. Para $l=0$, el resultado se tiene por el ítem 1. Para $1 \leq l<\omega$, por definición 1.5 ítem 2, $\mathcal{P}_{I}^{(l)}(O b j)=\bigcup\left\{\mathcal{O}_{I}(O b j)(a) \mid a \in T^{(l)}(I)\right\}$, así, por el ítem 2 de esta proposición,

$$
\mathcal{P}_{I}^{(l)}(O b j) \subset \bigcup\left\{\mathcal{O}\left(O b j_{i}\right)\left(\mathcal{S}_{I}(a)\right) \mid a \in T^{(l)}(I),\right.
$$

luego por la proposición 2.4 ítem 1 y la definición 1.3

$$
\mathcal{P}_{I}^{(l)}(O b j) \subset \bigcup\left\{\mathcal{O}\left(O b j_{i}\right)(a) \mid a \in T^{(l)}(\{i\}) .\right.
$$

Por lo tanto $\mathcal{P}_{I}^{(l)}(O b j) \subset \mathcal{P}^{(l)}\left(O b j_{i}\right)$.

7. Por definición 1.5 ítem $4 \mathcal{E}_{I}(O b j) \subset \bigcup\left\{\mathcal{O}_{I}(O b j)(a) \mid a \in T_{I}\right\}$. Luego, por el ítem 2 de esta proposición

$$
\bigcup\left\{\mathcal{O}\left(O b j_{i}\right)\left(\mathcal{S}_{I}(a)\right) \mid a \in T_{I}\right\} \subset \bigcup\left\{\mathcal{O}\left(O b j_{i}\right)(a) \mid a \in T\right\}=\mathcal{E}\left(O b j_{i}\right) .
$$

Obteniendo el resultado.

8. Sea $R \in \mathcal{P}_{I}^{(l)}(O b j)$, claramente, por definición 1.7,

$R=\left(\mathcal{R e l}\left(\mathcal{O}_{I}\right)(R), l\right)$. Del mismo modo tenemos que

$R=(\mathcal{R e l}(\mathcal{O})(R), l)$, dado el ítem 2 y 6 de esta proposición.

Por lo tanto $\operatorname{Rel}\left(\mathcal{O}_{I}\right)(R)=\operatorname{Rel}(\mathcal{O})(R)$.

La definición siguiente está basada en la definición 1.9. Además fijamos los tipos $T_{I}$ y tomamos $a \in T_{I}$

Definición 2.6. Sea $\mu$ una estructura múltiple en $O b j$, y sea $l \in \mathbb{N}^{0}$. Definimos:

1. Los relatores $\delta_{\mu}^{T}$ como

$$
\delta_{\mu}^{T}(a)=\left\{\begin{array}{cl}
\left(U^{a}(\mu), \operatorname{ord}_{I}(a)+1\right) & , \text { si, } U^{a}(\mu) \neq \emptyset \\
\emptyset^{\omega} & , \text { en otro caso. }
\end{array}\right.
$$

\section{La escala análoga de $\mu$ de orden $l$}

$$
\mathcal{A}_{\mu}^{l}=\left\{\delta_{\mu}^{T}(a) \mid \operatorname{ord}_{I}(a)=l \wedge \delta_{\mu}^{T}(a) \neq \emptyset^{\omega}\right\} .
$$

Manuscrito - Rev. Int. Fil., Campinas, v. 38, n.2, pp.89-154, jul.-ago. 2015. 


\section{La escala análoga total de $\mu$}

$$
\mathcal{A}_{\mu}=\bigcup\left\{\mathcal{A}_{\mu}^{l} \mid 0 \leq l<\operatorname{ord}_{I}(\mu)\right\} .
$$

4. $\mu_{\mathcal{A}}$ como una función de $\mu_{\mathcal{A}}: \mathbb{N}^{0} \cup\{\omega\} \mapsto \mathcal{E}\left(O b j_{i}\right)$, de modo que cumple

$$
\mu_{\mathcal{A}}(l)=\left\{\begin{array}{cl}
\mu(0) & \text { si, } l=0 \\
\mu(l) \cup \mathcal{A}_{\mu}^{l-1} & \text { si, } 1 \leq l<\omega, y, l \leq \operatorname{ord}_{I}(\mu) \\
\emptyset & \text { si, } 1 \leq l<\omega, y, l>\operatorname{ord}_{I}(\mu) \\
\emptyset^{\omega} & \text { si } l=\omega .
\end{array}\right.
$$

\section{Llamaremos a $\mu_{\mathcal{A}}$ la estructura análoga de $\mu$.}

Mostraremos mas adelante ${ }^{17}$, que $\mu_{\mathcal{A}}$ es muy útil al momento de reducir semánticamente, $\mu$ estructura múltiple, a una estructura simple.

Notemos que $\mu_{\mathcal{A}}$ es realmente una estructura. Tal resultado esta dado esencialmente por la proposición 2.5 ítems 3,6 y el siguiente hecho justificado mas adelante ${ }^{18}$

$$
\operatorname{Rel}(\mathcal{O})\left(\delta_{\mu}^{T}(a)\right) \subset U^{a}(\mu) \subset U^{\mathcal{S}_{I}(a)}\left(\mu_{\mathcal{A}}\right) .
$$

La idea de esta definición es reducir de manera conveniente las estructuras de varios individuos. Para ello lo que se ha hecho es agregar relatores convenientes para no perder la expresividad de $\mu$. Estos relatores son los $\delta_{\mu}^{T}(a)$, y para que sean realmente útiles necesitamos que sean distintos de $\emptyset^{\omega}$. Es por ello que construimos $\mathcal{A}_{\mu}^{l}$, para poder discriminar aquellos $\delta_{\mu}^{T}(a)$ que son $\emptyset^{\omega}$. Por último los agregamos a la estructura, de un solo individuo, considerando el orden de los relatores $\delta_{\mu}^{T}(a)$.

Ejemplo 2.7. Usando el ejemplo 2.2, partes 3 y 4, tenemos

1. Para el ítem 3, usando la definición anterior, $\mu_{\mathcal{A}}$ es de la forma

a) $\mu_{\mathcal{A}}(0)=(D \cup \mathbb{P}(D)) \times\{0\}$.

\footnotetext{
${ }^{17}$ En especifico desde 3.13 en adelante.

${ }^{18}$ Ver proposición 2.8 ítem 4.
} 
b) $\mu_{\mathcal{A}}(1)=\left\{\mathfrak{T}_{D}^{1}, \in_{D}^{1}, D^{1}, \mathbb{P}^{1}(D)\right\}$.

c) $\mu_{\mathcal{A}}(l)=\emptyset$, para $1<l<\omega$.

Donde $D^{1}=(D \times\{0\}, 1), y, \mathbb{P}(D)^{1}=(\mathbb{P}(D) \times\{0\}, 1)$.

2. Para el ítem 4, usando la definición anterior, $\mu_{\mathcal{A}}$ es de la forma

a) $\mu_{\mathcal{A}}(0)=\left(D \cup \mathbb{P}(D) \cup \mathbb{R}^{n}, \mathbb{P}\left(\mathbb{R}^{n}\right)\right) \times\{0\}$.

b) $\mu_{\mathcal{A}}(1)=\left\{\mathfrak{T}_{D}^{1}, \in_{D}^{1}, D^{1}, \mathbb{P}(D)^{1},\left(\mathbb{R}^{n}\right)^{1}, \mathbb{P}\left(\mathbb{R}^{n}\right)^{1}\right\}$.

c) $\mu_{\mathcal{A}}(l)=\emptyset$, para $1<l<\omega$.

Donde $D^{1}, \mathbb{P}(D)^{1}$ son definidos en el item anterior, $y\left(\mathbb{R}^{n}\right)^{1}=$ $\left(\mathbb{R}^{n} \times\{0\}, 1\right), \mathbb{P}(D)^{1}=\left(\mathbb{P}\left(\mathbb{R}^{n}\right) \times\{0\}, 1\right)$.

En lo que sigue usaremos las nociones de las definiciones 1.9 y 2.6. Además tengamos en mente la proposición 2.5 ítem 2, puesto que ella es la que da sentido a lo que viene.

Proposición 2.8. Sean $R \in \mathcal{E}_{I}(O b j), a \in T_{I}$.

1. $\operatorname{ord}(R)=\operatorname{ord}_{I}(R)$.

2. $U\left(\mu_{\mathcal{A}}\right)=U(\mu) \cup \mathcal{A}_{\mu}$.

3. $\operatorname{ord}\left(\mu_{\mathcal{A}}\right)=\operatorname{ord}_{I}(\mu)$.

4. $U^{a}(\mu) \subset U^{\mathcal{S}_{I}(a)}\left(\mu_{\mathcal{A}}\right)$.

Demostración. Enumerando las demostraciones:

1. Por proposición 2.5 ítem 2 tenemos

$$
R \in \mathcal{O}_{I}(O b j)(a) \subset \mathcal{O}\left(O b j_{i}\right)(a),
$$

así, $\operatorname{ord}_{I}(R)=\operatorname{ord}_{I}(a), \mathrm{y}, \operatorname{ord}(R)=\operatorname{ord}\left(\mathcal{S}_{I}(a)\right)$. Como $\operatorname{ord}_{I}(a)=$ $\operatorname{ord}\left(\mathcal{S}_{I}(a)\right)$, dada la proposición 2.4 ítem 1 , tenemos que

$$
\operatorname{ord}_{I}(R)=\operatorname{ord}(R) \text {. }
$$


2. Notemos que por definición 2.6 tenemos $\mu(l) \subset \mu_{\mathcal{A}}(l)$, para $1 \leq$ $l \leq \operatorname{ord}_{I}(\mu)$. Por definición 1.9 ítem 3 , y, el claro hecho $\mu(q) \neq \emptyset$ para $q>\operatorname{ord}_{I}(\mu)$, obtenemos

$$
U(\mu)=\bigcup\left\{\mu(j) \mid 1 \leq j \leq \operatorname{ord}_{I}(\mu)\right\} \subset U\left(\mu_{\mathcal{A}}\right) .
$$

Por otro lado, tenemos $\mathcal{A}_{\mu}^{l-1} \subset U\left(\mu_{\mathcal{A}}\right)$, para $0 \leq l \leq \operatorname{ord}_{I}(\mu)$. En síntesis

$$
U(\mu) \cup \mathcal{A}_{\mu} \subset U\left(\mu_{\mathcal{A}}\right) .
$$

Ahora sea $l \in \mathbb{N}^{0}$, tenemos que:

a) $l=0$, entonces, por definición 2.6, $\mu_{\mathcal{A}}(0)=\mu(0)$.

b) Si $0<l<\operatorname{ord}_{I}(\mu)$, luego $\mu_{\mathcal{A}}(l) \subset \mu(l) \cup \mathcal{A}_{\mu}^{l-1}$.

c) Si $l>\operatorname{ord}_{I}(\mu)$, luego $\mu_{\mathcal{A}}(l)=\emptyset$. Luego usando la definición 1.9 ítem 3 y eliminando los vacíos correspondientes,

$$
\begin{gathered}
U\left(\mu_{\mathcal{A}}\right)=\bigcup\left\{\mu_{\mathcal{A}}(0)\right\} \cup \bigcup\left\{\mu_{\mathcal{A}}(l) \mid 1 \leq l \leq \operatorname{ord}_{I}(\mu)\right\}, \\
\bigcup\left\{\mu_{\mathcal{A}}(0)\right\} \cup \bigcup\left\{\mu_{\mathcal{A}}(l) \mid 1 \leq l \leq \operatorname{ord}_{I}(\mu)\right\} \subset U(\mu) \cup \mathcal{A}_{\mu} .
\end{gathered}
$$

Por lo tanto $U\left(\mu_{\mathcal{A}}\right)=U(\mu) \cup \mathcal{A}_{\mu}$.

d) Por definición 2.1 y proposición 2.4 ítem 1

$$
\operatorname{ord}_{I}(\mu)=\left\{\begin{array}{cl}
\operatorname{máx}\{\operatorname{ord}(R) \mid R \in U(\mu)\} & , \text { si existe tal máximo. } \\
\omega & , \text { en otro caso. }
\end{array}\right.
$$

Usando la definición 2.6, tenemos, para $0 \leq l<\operatorname{ord}_{I}(\mu)$,

$$
x \in \mathcal{A}_{\mu}^{l} \subset \mathcal{A}_{\mu}, \mathrm{y}, \operatorname{ord}(x) \leq \operatorname{ord}_{I}(\mu),
$$

luego:

1) Si existe máx $\{\operatorname{ord}(R) \mid R \in U(\mu)\}$, entonces, por lo mostrado y el ítem anterior,

$$
\operatorname{máx}\{\operatorname{ord}(R) \mid R \in U(\mu)\}=\operatorname{máx}\left\{\operatorname{ord}(R) \mid R \in U(\mu) \cup \mathcal{A}_{\mu}\right\} \text {, }
$$$$
\operatorname{máx}\left\{\operatorname{ord}(R) \mid R \in U(\mu) \cup \mathcal{A}_{\mu}\right\}=\operatorname{máx}\left\{\operatorname{ord}(R) \mid R \in U\left(\mu_{\mathcal{A}}\right)\right\} \text {, }
$$

es decir

$$
\operatorname{máx}\{\operatorname{ord}(R) \mid R \in U(\mu)\}=\operatorname{máx}\left\{\operatorname{ord}(R) \mid R \in U\left(\mu_{\mathcal{A}}\right)\right\} .
$$


2) Si no existe máx $\{\operatorname{ord}(R) \mid R \in U(\mu)\}$, entonces, no existe máx $\left\{\operatorname{ord}(R) \mid R \in U\left(\mu_{\mathcal{A}}\right)\right\}$.

Usando los casos mostrados tenemos $\operatorname{ord}_{I}(\mu)=\left\{\begin{array}{cl}\operatorname{máx}\left\{\operatorname{ord}(R) \mid R \in U\left(\mu_{\mathcal{A}}\right)\right\} & , \text { si existe tal máximo. } \\ \omega & , \text { en otro caso. }\end{array}\right.$

Por lo tanto, por la definición 2.1, $\operatorname{ord}_{I}(\mu)=\operatorname{ord}\left(\mu_{\mathcal{A}}\right)$.

e) Sea $a \in T_{I}$, de modo que $\operatorname{ord}_{I}(a)=l$. Notemos que $\mu(l) \subset$ $\mu_{\mathcal{A}}(l)$, luego, usando las definiciones 1.9 ítem 2 y 2.3 , además de las proposiciones 2.4 ítem 1 y 2.5 ítem 2

$$
\begin{aligned}
& U^{a}(\mu)=\mu(l) \cap \mathcal{O}_{I}(O b j)(a) \subset \mu_{\mathcal{A}}(l) \cap \mathcal{O}\left(O b j_{i}\right)\left(\mathcal{S}_{I}(a)\right), \\
& \mu(l) \cap \mathcal{O}_{I}(O b j)(a) \subset \mu_{\mathcal{A}}(l) \cap \mathcal{O}\left(O b j_{i}\right)\left(\mathcal{S}_{I}(a)\right)=U^{\mathcal{S}_{I}(a)}\left(\mu_{\mathcal{A}}\right) . \\
& \text { Obteniendo } U^{a}(\mu) \subset U^{\mathcal{S}_{I}(a)}\left(\mu_{\mathcal{A}}\right) .
\end{aligned}
$$

\section{Estructura I fundamental}

El propósito de esta sección es explicitar cómo realizar una reducción de orden para estructuras.

Definición 2.9. Sean Obj una colección objetiva de $\{i\}$ y $T$ el conjunto de tipos de $\{i\}$. Definimos

1. $I_{T}$ como $I_{T}=\{\{\mathbb{N}\} \times\{a\} \mid a \in T\}$. Por mera estética denotaremos $\{\mathbb{N}\} \times\{a\}$ por $a^{T}$.

2. $O \mathrm{Obj}_{T}$ como $O b j_{T}=\{\mathcal{O}(O b j)(a) \mid a \in T\}$.

Proposición 2.10. Sea $a \in T$ se cumple

1. $T_{I_{T}}$ es un conjunto de tipos de $I_{T}$.

2. $\mathrm{Obj}_{T}$ es una colección objetiva de $I_{T}$.

3. $\mathcal{O}_{I_{T}}\left(O b j_{T}\right)\left(a^{T}\right)=\mathcal{O}(O b j)(a) \times\{0\}$.

Demostración. Enumerando las demostraciones: 
1. Sea $x \in \mathcal{G}^{(l)}\left(I_{T}\right)$ y $l \in \mathbb{N}$ arbitrario, si $x$ es considerado como una función desde un conjunto finito a $\mathcal{G}^{(l-1)}\left(I_{T}\right)$, es decir $x \subset$ $\mathbb{N} \times \mathcal{G}^{(l-1)}\left(I_{T}\right)$. Si $x=\{\mathbb{N}\} \times\{a\} \in I_{T}$, tendríamos $\{\mathbb{N}\} \times\{a\} \subset$ $\mathbb{N} \times \mathcal{G}^{(l-1)}\left(I_{T}\right)$, lo cual es una clara contradicción, puesto que $\mathbb{N} \notin \mathbb{N}$. Luego $T_{S} \cap \mathcal{G}^{(l)}\left(I_{T}\right)=\emptyset$, para cualquier $l \in \mathbb{N}$. Por lo tanto $T_{I_{T}}$ es un conjunto de tipos de $I_{T}$, dada la definición 1.1 ítem 2.

2. Notemos que $O b j_{T}$ es equipotente con $T$, razón por la cual $I_{T}$ es un conjunto ${ }^{19}$. Tal hecho esta justificado por la construcción de $O b j_{T}$ y el hecho que $\mathcal{O}(O b j)$ es inyectiva. Por otro lado notemos que por definición anterior $I_{T}$ es equipotente con $T$, razón por la cual $I_{T}$ es un conjunto ${ }^{20}$. Como $T$ es equipolente con $I_{T}$ y $O b j_{T}$, entonces $I_{T}$ es equipotente con $O b j_{T}$.

Ahora notemos que para $x \in O b j_{T}$, entonces $x=\mathcal{O}(O b j)(a)$, para algún $a \in T$, luego $x \neq \emptyset$ dada la definición 1.5 ítem 2 y la proposición 1.6 ítem 1 . Notemos que para $a, b \in T_{I}$ distintos entre si, se tiene por la proposición 1.6 ítem $3, \mathcal{O}(O b j)(a) \cap \mathcal{O}(O b j)(b)=$ $\emptyset$. Por lo tanto, por definición 1.5 ítem $1, \mathrm{Obj}_{T}$ es una colección objetiva de $I_{T}$.

3. Notemos que $I_{T}$ y $T$ son equipolentes. Fijemos entonces, $f$, la biyección entre $I_{T}$ y $T$, de modo que $f\left(a^{T}\right)=a$. Por otro lado veamos que la biyección entre $T$ es $O b j_{T}$ es $\mathcal{O}(O b j)$, así, la biyección entre $I_{T}$ y $O b j_{T}$, es, $\mathcal{O}(O b j) \circ f$. Luego, por definición 1.5 ítem 2 ,

$$
\mathcal{O}_{I_{T}}\left(O b j_{T}\right)(a)=\left(\mathcal{O}(O b j) \circ f\left(a^{T}\right)\right) \times\{0\}=\mathcal{O}(O b j)(a) \times\{0\} .
$$

Definición 2.11. Sea e una estructura simple, $a=\left\langle a_{1}, \ldots, a_{m}\right\rangle$ con $1 \leq m<\omega, \operatorname{ord}(a)=n \in \mathbb{N}$, y $R \in \mathcal{O}(\operatorname{Obj})(a)$. Definimos ${ }^{21}$ :

1. La reducción $\mathrm{I}$ de a de e, denotada por $\mathrm{I}_{e}(a)$ el conjunto que mostramos a continuación:

$$
\left\{\left((R, 0),\left(b_{1}, 0\right), \ldots,\left(b_{m}, 0\right)\right) \mid\left(b_{1}, \ldots, b_{m}\right) \in \operatorname{Rel}(\mathcal{O})(R) \wedge R \in U^{a}(e)\right\} .
$$

\footnotetext{
${ }^{19}$ Para mas detalle ver la pagina 234, teorema 8.7 [4].

${ }^{20}$ Para mas detalle ver la pagina 234, teorema 8.7 del libro [4].

${ }^{21}$ Por si las dudas, revisar definición 1.7 ítem 3.
} 
2. Delta I de a en $e$

$$
\delta_{e}^{\mathrm{I}}(a)=\left\{\begin{array}{cl}
\left(\mathrm{I}_{e}(a), 1\right) & , \text { si, } \mathrm{I}_{e}(a) \neq \emptyset \\
\emptyset^{\omega} & , \text { en otro caso. }
\end{array}\right.
$$

\section{Relación I fundamental de $e$}

$$
\mathrm{I}_{e}=\left\{\delta_{e}^{\mathrm{I}}(a) \mid a \in T_{I} \wedge \delta_{e}^{\mathrm{I}}(a) \neq \emptyset^{\omega}\right\}
$$

4. $e_{\mathrm{I}}$ como una función $e_{I}: \mathbb{N}^{0} \cup\{\omega\} \mapsto \mathcal{E}_{T_{I_{T}}}\left(O b j_{T}\right)$, de modo que

$$
e_{\mathrm{I}}(l)=\left\{\begin{array}{cl}
U(e) \times\{0\} & , \text { si,l=0 } \\
\mathrm{I}_{e} & , \text { si, } l=1 \\
\emptyset & , \text { si, } l>1 \\
\emptyset^{\omega} & , \text { si, } l=\omega .
\end{array}\right.
$$

\section{Llamaremos a $e_{\mathrm{I}}$ la estructura I fundamental de e.}

Notemos que esta estructura es múltiple pero de orden a lo mas 1 . $\mathrm{Su}$ propósito es reducir las estructuras de orden mayor a estructuras de orden 1. Sin embargo tal utilidad será mostrada a partir de definición 3.13 , en la reducción semántica de estructuras de orden mayor.

Esta es una formalización de los relatores $E_{t}$ definidos en el trabajo de Newton da Costa[1] página 9. Esta formalización es necesaria pues es posible deducir de su definición original que $E_{T}$ posee mas de un tipo.

Por cierto, el hecho de que la definición mostrada sea una buena definición, esta dado sistemáticamente por la proposición 2.10.

Proposición 2.12. Sean ord $(a)=n \geq 1$, de modo que: $a=\left\langle a_{1}, \ldots, a_{m}\right\rangle, y, b \in T$. Se cumple

1. Si $\delta_{e}^{\mathrm{I}}(a) \neq \emptyset^{\omega}$, entonces $\delta_{e}^{\mathrm{I}}(a) \in \mathcal{O}_{I_{T}}\left(O b j_{T}\right)\left(\left\langle a^{T}, a_{1}^{T}, \ldots, a_{m}^{T}\right\rangle\right)$. Es decir $\delta_{e}^{\mathrm{I}}(a)$ es de tipo $\left\langle a^{T}, a_{1}^{T}, \ldots, a_{m}^{T}\right\rangle$.

2. Si $\delta_{e}^{\mathrm{I}}(a) \neq \emptyset^{\omega}$, entonces $\operatorname{ord}_{I_{T}}\left(\delta_{e}^{\mathrm{I}}(a)\right)=1$.

3. $U\left(e_{\mathrm{I}}\right)=(U(e) \times\{0\}) \cup \mathrm{I}_{e}$

4. $\operatorname{ord}_{I_{T}}\left(e_{\mathrm{I}}\right)= \begin{cases}0 & , \text { si, } \mathrm{I}_{e}=\emptyset \\ 1 & , \text { en otro caso. }\end{cases}$ 
5. $U^{b}(e) \times\{0\}=U^{b^{s}}\left(e_{\mathrm{I}}\right)$.

6. $e_{\mathrm{I}}$ es una estructura.

Demostración. Enumerando las demostraciones:

1. Veamos que

$$
\mathrm{I}_{e}(a) \subset\left(U^{a}(e) \times\{0\}\right) \times\left(U^{a_{1}}(e) \times\{0\}\right) \times \ldots \times\left(U^{a_{n}}(e) \times\{0\}\right),
$$

Si $\delta_{e}^{\mathrm{I}}(a) \neq \emptyset^{\omega}$, entonces $\mathrm{I}_{e}(a) \neq \emptyset$, así $\mathrm{I}_{e}(a)$ es subconjunto

$$
\left(\mathbb{P}\left((\mathcal{O}(O b j)(a) \times\{0\}) \times\left(\mathcal{O}(O b j)\left(a_{1}\right) \times\{0\}\right) \times \ldots \times\left(\mathcal{O}(O b j)\left(a_{n}\right) \times\{0\}\right)\right)\right) \backslash\{\emptyset\},
$$

Así, por proposición 2.10 ítem 3,

$\mathrm{I}_{e}(a) \subset\left(\mathbb{P}\left(\mathcal{O}_{I_{T}}\left(O b j_{T}\right)\left(a^{T}\right) \times \mathcal{O}_{I_{T}}\left(O b j_{T}\right)\left(a_{1}^{T}\right) \times \ldots \times \mathcal{O}_{I_{T}}\left(O b j_{T}\right)\left(a_{n}^{T}\right)\right)\right) \backslash\{\emptyset\}$.

Por otro lado, por proposición 1.4 y definición 2.9 ítem 1, tenemos $\operatorname{ord}_{I_{T}}\left(\left\langle a^{T}, a_{1}^{T}, \ldots, a_{m}^{T}\right\rangle\right)=\operatorname{máx}\left\{\operatorname{ord}_{I_{T}}(b) \mid b \in\left\{a^{T}, a_{1}^{T}, \ldots, a_{n}^{T}\right\}\right\}+1$ es decir $\operatorname{ord}_{I_{T}}\left(\left\langle a^{T}, a_{1}^{T}, \ldots, a_{m}^{T}\right\rangle\right)=1$. Por lo tanto, por definición 1.5 ítem 2 ,

$$
\delta_{e}^{\mathrm{I}}(a)=\left(\mathrm{I}_{e}(a), 1\right) \in \mathcal{O}_{I_{T}}\left(O b j_{T}\right)\left(\left\langle a^{T}, a_{1}^{T}, \ldots, a_{m}^{T}\right\rangle\right) .
$$

2. Consecuencia clara del ítem anterior y la definición 1.10,

$$
\operatorname{ord}_{I_{T}}\left(\delta_{e}^{\mathrm{I}}(a)\right)=\operatorname{ord}_{I_{T}}\left(\left\langle a^{T}, a_{1}^{T}, \ldots, a_{m}^{T}\right\rangle\right)=1 .
$$

3. Veamos que, por la definiciones 1.9 ítem $3,2.11$, ítem 5,

$$
\begin{aligned}
& U\left(e_{I}\right)=\bigcup e_{\mathrm{I}}\left[\mathbb{N}^{0}\right]=\bigcup e_{\mathrm{I}}[\{0,1\}]=\bigcup\left\{U(e) \times\{0\}, \mathrm{I}_{e}\right\}, \\
& \bigcup e_{\mathrm{I}}[\{0,1\}]=\bigcup\left\{U(e) \times\{0\}, \mathrm{I}_{e}\right\}=(U(e) \times\{0\}) \cup \mathrm{I}_{e},
\end{aligned}
$$

es decir $U\left(e_{I}\right)=(U(e) \times\{0\}) \cup \mathrm{I}_{e}$. 
4. De la definición 1.10

$$
\operatorname{ord}_{I_{T}}\left(e_{\mathrm{I}}\right)=\left\{\begin{array}{cl}
\operatorname{máx}\left\{\operatorname{ord}_{I_{T}}(R) \mid R \in U\left(e_{\mathrm{I}}\right)\right\} & , \text { si existe tal máximo. } \\
\omega & \text {, en otro caso. }
\end{array}\right.
$$

del ítem anterior tenemos, fijando $p=\operatorname{máx}\left\{\operatorname{ord}_{I_{T}}(R) \mid R \in(U(e) \times\right.$ $\left.\{0\}) \cup \mathrm{I}_{e}\right\}$ por motivos tipográficos,

$\operatorname{ord}_{I_{T}}\left(e_{\mathrm{I}}\right)=\left\{\begin{array}{cl}\operatorname{máx}\left\{\operatorname{ord}_{I_{T}}(R) \mid R \in(U(e) \times\{0\}) \cup \mathrm{I}_{e}\right\} & , \text { si existe, } p \\ \omega & \text { en otro caso. }\end{array}\right.$

Claramente existe máx $\operatorname{ord}_{I_{T}}(R) \mid R \in(U(e) \times\{0\}) \cup \mathrm{I}_{e}$, dado el ítem 2 de esta proposición. Luego $\operatorname{ord}_{I_{T}}\left(e_{\mathrm{I}}\right)=\operatorname{máx}\left\{\operatorname{ord}_{I_{T}}(R) \mid R \in\right.$ $\left.(U(e) \times\{0\}) \cup \mathrm{I}_{e}\right\}$, es decir,

$\operatorname{ord}_{I_{T}}\left(e_{\mathrm{I}}\right)=\left\{\begin{array}{cl}\operatorname{máx}\left\{\operatorname{ord}_{I_{T}}(R) \mid R \in(U(e) \times\{0\}) \cup \mathrm{I}_{e}\right\} & , \text { si, } \mathrm{I}_{e} \neq \emptyset \\ \operatorname{máx}\left\{\operatorname{ord}_{I_{T}}(R) \mid R \in U(e) \times\{0\}\right\} & , \text { en otro caso. }\end{array}\right.$

Dada la definición 1.10 y el ítem 2 de esta proposición, claramente tenemos

$$
\operatorname{ord}_{I_{T}}\left(e_{\mathrm{I}}\right)= \begin{cases}1 & , \text { si, } \mathrm{I}_{e} \neq \emptyset \\ 0 & , \text { en otro caso. }\end{cases}
$$

5. Por definición 1.9 ítem $2 U^{b}(e)=e(\operatorname{ord}(b)) \cap \mathcal{O}(O b j)(b)$. Luego por la proposición 1.6 ítem 5

$$
\begin{gathered}
U^{b}(e) \times\{0\}=(e(\operatorname{ord}(b)) \cap \mathcal{O}(O b j)(b)) \times\{0\}, \\
(e(\operatorname{ord}(b)) \cap \mathcal{O}(O b j)(b)) \times\{0\}=(U(e) \times\{0\}) \cap(\cap \mathcal{O}(O b j)(b) \times\{0\}),
\end{gathered}
$$

así, por las definiciones 1.9 ítem 2, 2.11 ítem 4, y la proposición 2.10 ítem 3 ,

$$
U^{b}(e) \times\{0\}=e_{\mathrm{I}}(0) \cap \mathcal{O}_{I_{T}}\left(O b j_{T}\right)\left(b^{s}\right)=U^{b^{s}}(e) .
$$

6. Para empezar, notemos que $e_{\mathrm{I}}$ es una pre-estructura, tal hecho se justifica en el item 1 de esta proposición, de lo que se obtiene por definición 1.5

$$
\mathrm{I}_{e} \subset \mathcal{P}_{I_{T}}^{(1)}\left(O b j_{T}\right)
$$


Por otro lado, los relatores $\delta_{e}^{I}(a)$ cumplen la siguiente igualdad $\operatorname{Rel}\left(\mathcal{O}_{I_{T}}\right)\left(\delta_{e}^{I}(a)\right) \subset\left(U^{a}(e) \times\{0\}\right) \times\left(U^{a_{1}}(e) \times\{0\}\right) \times \ldots \times\left(U^{a_{n}}(e) \times\{0\}\right)$, donde $a=\left\langle a_{1}, \ldots, a_{n}\right\rangle$. Usando el ítem anterior tenemos que

$$
\operatorname{Rel}\left(\mathcal{O}_{I_{T}}\right)\left(\delta_{e}^{I}(a)\right) \subset U^{a^{T}}\left(e_{\mathrm{I}}\right) \times U^{a_{1}^{s}}(e) \times \ldots \times U^{a_{n}^{s}}\left(e_{\mathrm{I}}\right) .
$$

Luego, dado que se cumplen las clausulas de la definición 1.9 ítem 5 , tenemos que $e_{\mathrm{I}}$ es una estructura.

En lo que sigue, mostraremos una estructura que pretende ser el símil de $\varepsilon(D)$ mostrado en el trabajo de Newton da Costa[1] página 4.

\section{Estructura completa}

Definición 2.13. Sea Obj una colección objetiva de un conjunto I, definimos la estructura completa $\Omega_{I}$, como una función de la forma $\Omega_{I}: \mathbb{N}^{0} \cup\{\omega\} \mapsto \mathcal{E}_{I}(O b j)$, que cumple

$$
\Omega_{I}(l)=\mathcal{P}_{I}^{(l)}(O b j), \text { para } l \in \mathbb{N}^{0}, y, \Omega_{I}(\omega)=\emptyset^{\omega} .
$$

No es difícil ver que lo mostrado corresponde a una buena definición, es decir $\Omega_{I}$ es una estructura. Primero, es obvio que es una pre-estructura. Segundo, notar que de la construcción inductiva de 1.5 tenemos que $R \in \mathcal{E}_{I}(O b j)$ entonces

$$
\operatorname{Rel}\left(\mathcal{O}_{I}\right)(R) \subset \mathcal{O}_{I}(O b j)\left(a_{1}\right) \times \ldots \mathcal{O}_{I}(O b j)\left(a_{n}\right),
$$

usando el hecho que es una pre-estructura y la proposición que se encuentra a continuación, parte 2 , tenemos que $\Omega_{I}$ es una estructura.

Proposición 2.14. Sean a $\in T_{I}$, Obj una colección objetiva de $I, y$ sea $\Omega_{I}$ la estructura completa. Se cumple:

1. $U\left(\Omega_{I}\right)=\mathcal{E}_{I}(O b j)$.

2. $U^{a}\left(\Omega_{I}\right)=\mathcal{O}_{I}(O b j)(a)$.

Demostración. Enumerando las demostraciones: 
1. Claramente por definición 1.9 ítem 3

$$
\Omega_{I}\left[\mathbb{N}^{0}\right]=\left\{\mathcal{P}_{I}^{(l)}(O b j) \mid 0 \leq l<\omega\right\},
$$

luego deducimos, usando la definición 1.5

$$
U\left(\Omega_{I}\right)=\bigcup\left\{\mathcal{O}_{I}(O b j)(a) \mid a \in T_{I}\right\}=\mathcal{E}_{I}(O b j)
$$

2. Sea $a \in T^{(l)}(I)$, para $l \in \mathbb{N}^{0}$, luego, por proposición 1.5 ítem 2 y definición de $\Omega_{I}$,

$$
\begin{gathered}
\mathcal{O}_{I}(O b j)(a)=\mathcal{O}_{I}(O b j)(a) \cap \mathcal{P}_{I}^{(l)}(O b j)=\mathcal{O}_{I}(O b j)(a) \cap \Omega_{I}(l), \\
\mathcal{O}_{I}(O b j)(a) \cap \Omega_{I}(l)=U^{a}\left(\Omega_{I}\right),
\end{gathered}
$$

es decir, $\mathcal{O}_{I}(O b j)(a)=U^{a}\left(\Omega_{I}\right)$.

Por cierto cuando $I$ sea $\{i\}$, denotaremos $\Omega_{I}$, como $\Omega$.

\section{Lenguajes y semántica para estructuras.}

\section{Lenguajes formales para tipos.}

Definición 3.1. Sean $T_{I}$ un conjunto de tipos, $m$ un número ordinal, $1 \leq m \leq \omega, \Gamma$ un conjunto cualquiera. Definimos los siguientes conjuntos:

\section{Conectivos.}

$$
\mathcal{C}=\{\rightarrow, \wedge, \vee, \neg, \leftrightarrow\},
$$

donde: $\rightarrow$ es la implicación, $\wedge$ la conjunción $\vee$ la disyunción, $\neg$ la negación, $\leftrightarrow$ la equivalencia.

2. Cuantificadores.

$$
\mathcal{Q}=\{\exists, \forall\},
$$

donde: $\exists$ significa existe $y \forall$, para todo. 
3. Variables. Para esto tenemos que definir un conjunto $\mathcal{V}_{a}$, para cada a $\in T_{I}$. Por lo general exigiremos que tal conjunto sea numerable, o bien vacío, dependiendo del caso. También exigiremos que para $a \neq b$, con $a, b \in T_{I}, \mathcal{V} a r_{a} \cap \mathcal{V} a r_{b}=\emptyset$. Lo denotaremos, en caso de ser distinto del vació

$$
\operatorname{Var}_{a}=\left\{x_{i}^{a} \mid 1 \leq i<\omega\right\}
$$

$\mathcal{V}_{a} r_{a}$ sera llamado conjunto de variables de tipo a. Luego definimos:

$$
\mathcal{V} a r_{T_{I}}^{m}=\bigcup\left\{\mathcal{V} a r_{a} \mid a \in T_{I} \wedge \operatorname{ord}_{I}(a)<m\right\}
$$

Este será el conjunto de variables del lenguaje de orden $m$.

4. Constantes. Para este caso tenemos que definir un conjunto Const $_{a}$, para $a \in T_{I}$. Exigiremos que para $a, b \in T_{I}$, distintos, se tenga Const $_{a} \cap \mathcal{C}$ onst $_{b}=\emptyset$. Por cierto Const sera llamado constantes de tipo a. Además necesitaremos un símbolo $R_{\emptyset}$, que cumpla: $R_{\emptyset} \notin$ Const $_{a}$, para cualquier $a \in T_{I}$, tal símbolo sera llamado relación vacía. Ahora estamos en condiciones de definir

$$
\operatorname{Const}_{T_{I}}^{m}=\bigcup\left\{\mathcal{C} \text { onst }_{a} \mid a \in T_{I} \wedge \operatorname{ord}_{I}(a) \leq m\right\} \cup\left\{R_{\emptyset}\right\} .
$$

Este será el conjunto de constantes del lenguaje $e^{22}$.

5. Igualdades. construiremos un conjunto de simbolos arbitrarios que representan la igualdad de tipo a, tales son $\stackrel{a}{=}$, para $a \in T_{I}$. Luego definimos

$$
\Xi_{T_{I}}^{m}=\left\{\stackrel{a}{=} \mid a \in T_{I} \wedge \mathcal{V} a r_{a} \neq \emptyset \wedge \operatorname{ord}_{I}(a)<m\right\}
$$

Este será el conjunto de igualdades del lenguaje.

6. Paréntesis. Este conjunto es el más sencillo, y es de la forma: $\mathcal{P}=\{()$,$\} .$

${ }^{22}$ Un detalle curioso es que este lenguaje tiene una constante que es la relación vacía. $\mathrm{Su}$ inclusión es debido a motivos prácticos.

Manuscrito - Rev. Int. Fil., Campinas, v. 38, n.2, pp.89-154, jul.-ago. 2015. 
Exigiremos que los conjuntos: $\mathcal{C}, \mathcal{Q}, \mathcal{V}_{T_{I}}^{m}$, $\mathcal{C}_{\text {onst }} T_{T_{I}}^{m}, \Xi_{T_{I}}^{m}$, y $\mathcal{P}$, sean disjuntos entre si. Además $\Gamma=$ Const $_{T_{I}}^{m} \backslash\left\{R_{\emptyset}\right\}$. Luego definimos $\mathcal{L}_{T_{I}}^{m}(\Gamma)$ de la forma

$$
\mathcal{L}_{T_{I}}^{m}(\Gamma)=\mathcal{C} \cup \mathcal{Q} \cup \mathcal{P} \cup \Xi_{T_{I}}^{m} \cup \mathcal{V} a r_{T_{I}}^{m} \cup \mathcal{C} \text { onst } T_{T_{I}}^{m} .
$$

Llamaremos a este conjunto lenguaje de individuos $I$ de orden $m$. Si $T_{I}=T$ lo nombraremos por lenguaje de orden $m$. Por ultimo definimos dos elementos mas:

1. Términos del lenguaje de tipo a de $\mathcal{L}_{T_{I}}^{m}(\Gamma)$, como

$$
\mathrm{T}^{a}\left(\mathcal{L}_{T_{I}}^{m}(\Gamma)\right)=\left\{\begin{array}{cl}
\text { Const }_{a} \cup \mathcal{V}_{a r} a & , \text { si, } \operatorname{ord}_{I}(a)<m \\
\text { Const }_{a} & , \text { si }_{\text {ord }}(a)=m
\end{array}\right.
$$

\section{Términos del lenguaje de $\mathcal{L}_{T_{I}}^{m}(\Gamma)$}

$$
\mathrm{T}\left(\mathcal{L}_{T_{I}}^{m}(\Gamma)\right)=\bigcup\left\{\mathrm{T}^{a}\left(\mathcal{L}_{T_{I}}^{m}(\Gamma)\right) \mid \operatorname{ord}_{I}(a) \leq m\right\} .
$$

Es un hecho clásico que se necesitan menos conectores y cuantificadores que los mostrados. En algunas partes de este trabajo se usaran las equivalencias: $\alpha \rightarrow \beta \equiv \neg \alpha \vee \beta, \alpha \leftrightarrow \beta \equiv \alpha \rightarrow \beta \wedge \beta \rightarrow \alpha$ y otras mas que serán mencionadas mas adelante, para reducir otras cosas.

Quizás sea pertinente explicar que este lenguaje no identifica, en principio, lo que es un relator de la manera clásica. Tal identificación está oculta en el tipo y en la construcción de las formulas atómicas. Por ejemplo, si $I=\{i\}$ y $R$ es una constante de tipo $\langle i, i\rangle$, consideraremos $R\left(x_{1}^{i}, x_{2}^{i}\right)$, con $x_{1}^{i}, x_{2}^{i}$ variables de tipo $i$, una formula atómica, en tal caso notemos que $R$ es visto como una relación. Con respecto al ejemplo mencionado podemos ver que si $R^{\prime}$ es una constante de tipo $\langle\langle i, i\rangle\rangle$, entonces $R^{\prime}(R)$ es un formula atómica. Dicho de manera general, el tipo es el que esconde el papel que juega la constante, que bien puede ser de un relator, o de objeto dependiendo del caso. Por cierto este lenguaje no usará funciones de un modo clásico, puesto que tales serán consideradas como relaciones como en el ejemplo 1.8 .

Definición 3.2. Usando los términos definidos en 3.1 definimos los siguientes operadores: 
1. Construimos el operador $\mathfrak{F}^{=}$, de la forma:

$\mathfrak{F}^{=}\left(\mathcal{L}_{T_{I}}^{m}(\Gamma)\right)=\left\{x \stackrel{a}{=} y \mid \stackrel{a}{=} \in \Xi_{T_{I}}^{m} \wedge x, y \in \mathrm{T}^{a}\left(\mathcal{L}_{T_{I}}^{m}(\Gamma)\right) \wedge \operatorname{ord}_{I}(a)<m\right\}$.

Notemos que $x, y$ son variables del metalenguaje ${ }^{23}$ para términos de $\mathcal{L}_{T_{I}}^{m}(\Gamma)$.

2. Definiremos $\mathfrak{F}^{\Gamma}\left(\mathcal{L}_{T_{I}}^{m}(\Gamma)\right)$, de la forma:

$$
y \in \mathfrak{F}^{\Gamma}\left(\mathcal{L}_{T_{I}}^{m}(\Gamma)\right) \text {, si y solo si, } y=x_{0}\left(x_{1}, \ldots, x_{n}\right),
$$

donde:

$a_{0}=\left\langle a_{1}, \ldots, a_{n}\right\rangle, x_{0} \in \mathrm{T}^{a_{0}}\left(\mathcal{L}_{T_{I}}^{m}(\Gamma)\right) \cup\left\{R_{\emptyset}\right\}, x_{j} \in \mathrm{T}^{a_{j}}\left(\mathcal{L}_{T_{I}}^{m}(\Gamma)\right)$ para $1 \leq j \leq n, y, \operatorname{ord}_{I}\left(a_{0}\right) \leq m$. Como antes, $x_{0}, x_{1}, \ldots, x_{n}$ son variables del metalenguaje para términos de $\mathcal{L}_{T_{I}}^{m}(\Gamma)$.

Usando lo anterior definimos lo que realmente nos importa:

$$
\mathfrak{F}^{A}\left(\mathcal{L}_{T_{I}}^{m}(\Gamma)\right)=\mathfrak{F}^{=}\left(\mathcal{L}_{T_{I}}^{m}(\Gamma)\right) \cup \mathfrak{F}^{\Gamma}\left(\mathcal{L}_{T_{I}}^{m}(\Gamma)\right) .
$$

Llamaremos al conjunto $\mathfrak{F}^{A}\left(\mathcal{L}_{T_{I}}^{m}(\Gamma)\right)$ las formulas atómicas de $\mathcal{L}_{T_{I}}^{m}(\Gamma)$.

Definición 3.3. Sean $k, j$ cardinales distintos de 0 , sean $x, \alpha$ metavariables del lenguaje que representan sucesiones, en $j$, de variables y formulas, y sea $Z$ metavariable que representara alguno de los siguientes simbolos $\{\neq, \forall, \exists, \vee, \wedge\}$, introducimos la siguiente notación

1. Para $j<\omega$,

$$
\begin{gathered}
Z^{j} x_{j}=Z x_{0} \ldots Z x_{j-1}\left(\alpha_{1}\right), Z^{j}\left(\alpha_{j}\right)=Z\left(\alpha_{0}\right) \ldots Z\left(\alpha_{j-1}\right), y, \\
Z_{j}\left(\alpha_{j}\right)=\left(\alpha_{0}\right) Z \ldots Z\left(\alpha_{j-1}\right) .
\end{gathered}
$$

2. Para $j \geq \omega$,

$$
\begin{gathered}
Z^{j} x_{j}\left(\alpha_{1}\right)=Z x_{0} \ldots Z x_{\omega} \ldots\left(\alpha_{1}\right), Z^{j}\left(\alpha_{j}\right)=Z\left(\alpha_{0}\right) \ldots Z\left(\alpha_{\omega}\right) \ldots ; \\
Z_{j}\left(\alpha_{j}\right)=\left(\alpha_{0}\right) Z \ldots Z\left(\alpha_{\omega}\right) Z \ldots,
\end{gathered}
$$

de manera sencilla resumiremos el primero y el tercero como

$$
Z^{j} x_{j}\left(\alpha_{1}\right)=Z_{i \in j} x_{i}\left(\alpha_{1}\right) ; Z_{j}\left(\alpha_{j}\right)=Z_{i \in j} \alpha_{i},
$$

respectivamente.

\footnotetext{
${ }^{23}$ Son variables para la exposición, no para el lenguaje en sí.
}

Manuscrito - Rev. Int. Fil., Campinas, v. 38, n.2, pp.89-154, jul.-ago. 2015. 
Sean $A, B, C$ conjunto definamos los siguientes operadores:

1. El conjunto $\mathcal{B}^{k}(A, B, C)$ de la forma

$$
\begin{aligned}
& \mathcal{B}^{k}(A, B, C)= \\
& \left\{Z^{j} x_{j}\left(\alpha_{1}\right) \mid Z \in A \wedge 1 \leq j<k \wedge \text { para } 0 \leq i<j, x_{i} \in B \wedge \alpha_{1} \in C\right\}
\end{aligned}
$$

2. El conjunto $\mathcal{B}^{k}(A, C)$ de la forma

$$
\mathcal{B}^{k}(A, C)=\left\{Z^{j}\left(\alpha_{j}\right) \mid Z \in A \wedge 1 \leq j<k \wedge \text { para } 0 \leq i<j, \alpha_{i} \in C\right\} .
$$

3. Del mismo modo $\mathcal{B}_{k}(A, C)$ de la forma

$$
\mathcal{B}_{k}(A, C)=\left\{Z_{j}\left(\alpha_{j}\right) \mid Z \in A \wedge 2 \leq j<k \wedge \text { para } 0 \leq i<j, \alpha_{i} \in C\right\} .
$$

Esta definición es requerida para poder hablar de bloques de manera un tanto mas precisa.

Otra forma de introducir este lenguaje puede ser vista en [6], en especifico en el capitulo catorce.

Definición 3.4. En el lenguaje $\mathcal{L}_{T_{I}}^{m}(\Gamma)$, sean $A$ un conjunto cualquiera, $l$ un ordinal tal que $1 \leq l<\omega, q$ y $k$ cardinales, definimos el operador \langle\rangle$_{\mathfrak{F}}^{q k}$, de la forma:

$$
\left.\langle A\rangle_{\mathfrak{F}}^{q k}=\mathcal{B}^{2}(\{\neg\}, A) \cup \mathcal{B}^{q}\left(\{\forall\}, \mathcal{V} a r_{T_{I}}^{m}, A\right) \cup \mathcal{B}_{k}(\{\wedge\}, A\}\right) \cup A .
$$

Asi podemos definir de manera inductiva:

$$
\mathfrak{F}_{q k}^{1}\left(\mathcal{L}_{T_{I}}^{m}(\Gamma)\right)=\left\langle\mathfrak{F}^{A}\left(\mathcal{L}_{T_{I}}^{m}(\Gamma)\right)\right\rangle_{\mathfrak{F}}^{q k}, y, \mathfrak{F}_{q k}^{l+1}\left(\mathcal{L}_{T_{I}}^{m}(\Gamma)\right)=\left\langle\mathfrak{F}^{l}\left(\mathcal{L}_{T_{I}}^{m}(\Gamma)\right)\right\rangle_{\mathfrak{F}}^{q k} .
$$

Luego de toda esta vuelta definiremos el operador $\mathfrak{F}_{q k}\left(\mathcal{L}_{T_{I}}^{m}(\Gamma)\right)$, de la siguiente forma:

$$
\mathfrak{F}_{q k}\left(\mathcal{L}_{T_{I}}^{m}(\Gamma)\right)=\bigcup\left\{\mathfrak{F}_{q k}^{l}\left(\mathcal{L}_{T_{I}}^{m}(\Gamma)\right) \mid 1 \leq l<\omega\right\} .
$$

Tal conjunto sera llamado las formulas de $\mathcal{L}_{T_{I}}^{m}(\Gamma)$.

Esta definición permite formalizar el hecho de que Newton da Costa habla de formulas con un bloque infinito de disyuntores. 
Por cierto esta definición es completa, ya que cualquier fórmula que use la implicación, la equivalencia, la existencia, la disyunción, pueden ser reducidas usando las equivalencias: $\vee_{j} \alpha_{j} \equiv \neg\left(\wedge_{j}\left(\neg\left(\alpha_{j}\right)\right)\right.$, $\alpha \rightarrow \beta \equiv(\neg(\alpha)) \vee(\beta), \forall^{j} x_{j}(\alpha) \equiv \neg\left(\exists^{j} x_{j}(\neg(\alpha))\right), \mathrm{y}, \alpha \leftrightarrow \beta \equiv(\alpha \rightarrow$ $\beta) \wedge(\beta \rightarrow \alpha)$.

Dada esta explicación, veremos que las dos clases de lenguajes, que aparecen en el trabajo de Newton da Costa[1] página 5, corresponden al lenguaje $\mathcal{L}_{T_{I}}^{m}(\Gamma)$ cuya construcción de formulas puede ser interpretada de dos maneras. O bien como $\mathfrak{F}_{\omega \omega}\left(\mathcal{L}_{T_{I}}^{m}(\Gamma)\right)$, en tal caso solo anotaremos $\mathfrak{F}\left(\mathcal{L}_{T_{I}}^{m}(\Gamma)\right)^{24}$. O bien $\mathfrak{F}_{\omega \kappa}\left(\mathcal{L}_{T_{I}}^{m}(\Gamma)\right)^{25}$, donde claramente por la definición $\mathcal{B}_{\kappa}, \mathcal{B}^{\omega}$ son bloques de disyunciones de largo menor a $\kappa$, y, bloques de cuantificadores de largo menor $\omega$, respectivamente.

Definición 3.5. Sean $q, k$ cardinales, $y, \gamma \in \mathfrak{F}_{q k}\left(\mathcal{L}_{T_{I}}^{m}(\Gamma)\right)$. Definimos, para $a \in T_{I}, y, \operatorname{ord}_{I}(a)<\omega$

$$
\operatorname{var}_{a}^{\mathcal{L}}(\gamma)=\left\{x \in \mathcal{V} a r_{a} \mid x \text { aparece en } \gamma\right\},
$$

llamando variables de tipo a en $\gamma$, Además:

$$
\operatorname{var}^{\mathcal{L}}(\gamma)=\bigcup\left\{\operatorname{var}_{a}^{\mathcal{L}}(\gamma) \mid a \in T_{I}\right\},
$$

llamado variables en $\gamma$.

Uno podría considerar las formulas como sucesiones de símbolos en el lenguaje y ver si la variable pertenece a la imagen de esta sucesión. Pero tal grado de precisión me parece innecesario.

No es difícil ver que $\# \operatorname{var}_{a}^{\mathcal{L}}(\alpha) \leq \omega$, pero lo que no es siempre obvio es que $\# \operatorname{var}^{\mathcal{L}}(\alpha) \leq \omega$, puesto que es posible que $\# T_{I}>\omega \mathrm{y}$ $k>\omega$, lo que hace posible la aparición de una cantidad no numerable de variables.

\section{Semántica para estructuras.}

Algo previo a mencionar, para esta subsección, es que todos los resultados mostrados son obtenibles o igualmente definibles, tanto en

\footnotetext{
${ }^{24}$ Esta es equivalente a $L^{\omega}(R)$ en [1] página 5 .

${ }^{25}$ Esta es equivalente a $L_{\omega \kappa}^{\omega}(R)$ en [1] página 5 .
} 
estructuras como en pre-estructuras. Sin embargo el trabajo esta enfocado en estructuras y hablaremos desde ellas.

Además, en esta subsección, pensaremos que un modelo es una función que interpreta las constantes, de un lenguaje dado, en una estructura. Para luego dar a lugar a la interpretación de la formulas del lenguaje. Ciertamente el trabajo que viene está inspirado en la definición clásica de Tarski, pero toma en cuenta la estructura jerárquica de tipos de la teoría.

Definición 3.6. Sean $\mathcal{L}_{T_{I}}^{m}(\Gamma)$ un lenguaje y $\Psi$ una estructura. Definimos:

1. $\Psi$ permite $\mathcal{L}_{T_{I}}^{m}(\Gamma)$, si y solo si para todo $a \in T_{I}$, con ord $\operatorname{ord}_{I}(a)<$ $m: U^{a}(\Psi)=\emptyset$, entonces, $\mathcal{V} a r_{a}=\emptyset$.

2. Si $\Psi$ permite $\mathcal{L}_{T_{I}}^{m}(\Gamma)$, entonces: un modelo $M$ en $\Psi$ para

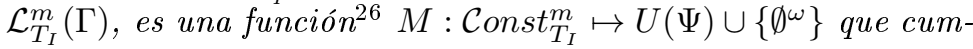
ple

a) $M$ es inyectiva.

b) Para todo $a \in T_{I}$, con $\operatorname{ord}_{I}(a) \leq m, M\left[\right.$ Const $\left._{a}\right] \subset U^{a}(\Psi)$.

c) $M\left(R_{\emptyset}\right)=\emptyset^{\omega}$

donde $M[]$ denota la imagen de un conjunto.

El ítem 1 de la definición 3.6 está especialmente pensado para que la aparición de una variable de tipo $a$ en una formula implique que hay objetos del cual hablar en $\Psi$, lo cual esta directamente relacionado con las siguientes definiciones donde se habla de la interpretación de formulas. Por lo general asumiremos que $\Psi$ permite $\mathcal{L}_{T_{I}}^{m}(\Gamma)$.

Definición 3.7. Sea $\Psi$ una estructura que permite $\mathcal{L}_{T_{I}}^{m}(\Gamma)$. Definimos

1. $v$ es una asignación de variable en $\Psi$ si y solo si $v$ es una función de la forma $v: \mathcal{V} a r_{T_{I}}^{m} \mapsto U(\Psi)$, que cumple

$$
x \in \mathcal{V} a r_{a} \text {, entonces, } v(x) \in U^{a}(\Psi) .
$$

\footnotetext{
${ }^{26}$ Recordar definición 3.1, en especifico el conjunto de constantes del lenguaje.
}

Manuscrito - Rev. Int. Fil., Campinas, v. 38, n.2, pp.89-154, jul.-ago. 2015. 
2. Sean $q$ es un cardinal, $j<q, x_{i}$ variables de tipo $a_{i}$ y $p_{i} \in$ $U^{a_{i}}(\Psi)$, para $i \in j$. Dada una asignación de variable $v$, diremos que la asignación de variables de $v_{x_{j}}^{p_{j}}$ es de la forma

$$
v_{x_{j}}^{p_{j}}(y)=\left\{\begin{array}{cl}
p_{i} & \text { si existe } i \in j \text { de modo que } x_{i}=y \\
v(y) & \text { en otro caso. }
\end{array}\right.
$$

Notemos que la definición es buena, es decir, no hay problemas con el hecho de que $v(x) \in U^{a}(\Psi)$, puesto que si $\mathcal{V}_{a r} \neq \emptyset$ tenemos que $U^{a}(\Psi) \neq \emptyset$, dado que $\Psi$ permite $\mathcal{L}_{T_{I}}^{m}(\Gamma)$. Claramente si $\mathcal{V}_{a} r_{a}=\emptyset$, hay menos de qué preocuparse.

Por cierto, en lo que sigue del trabajo, usaremos el símbolo $\equiv$ para denotar cadenas de símbolos iguales, pero siempre tener en mente que esta relación es en el metalenguaje.

Definición 3.8. Sean $\Psi$ una estructura, $M$ un modelo en $\Psi$ fijo para $\mathcal{L}_{T_{I}}^{m}(\Gamma)$ y v una asignación de variable en $\Psi$. Definimos:

1. Para $t \in \mathrm{T}\left(\mathcal{L}_{T_{I}}^{m}(\Gamma)\right)$ t denota a la asignación de variable $v$, como $\Psi(t)[v]$, que cumple

a) $\Psi(x)[v]=v(x)$ si $x$ es una variable de cualquier tipo.

b) $\Psi(c)[v]=M(c)$ si $c \in \Gamma$.

2. Para $\alpha \in \mathfrak{F}_{q k}\left(\mathcal{L}_{T_{I}}^{m}(\Gamma)\right) \Psi$ satisface la formula $\alpha$ respecto a la asignación de variable $v$, como $\Psi \models \alpha[v]$, a través de los siguientes criterios

- Para elementos en $\mathfrak{F}^{A}\left(\mathcal{L}_{T_{I}}^{m}(\Gamma)\right)$ :

a) Para $x \stackrel{a}{=} y$,

$$
\Psi \models x \stackrel{a}{=} y \text {, si } y \text { solo si, } \Psi(x)[v]=\Psi(y)[v] \text {. }
$$

donde $x, y \in \mathrm{T}^{a}\left(\mathcal{L}_{T_{I}}^{m}(\Gamma)\right), a \in T_{I}, \operatorname{ord}_{I}(a) \leq m$.

b) $\operatorname{Para} x_{0}\left(x_{1}, \ldots, x_{n}\right)$,

$$
\begin{aligned}
& \Psi \models x_{0}\left(x_{1}, \ldots, x_{n}\right)[v], \text { si y solo si, } \\
& \quad\left(\Psi\left(x_{1}\right)[v], \ldots, \Psi\left(x_{n}\right)[v]\right) \in \operatorname{Rel}\left(\mathcal{O}_{I}\right)\left(\Psi\left(x_{0}\right)[v]\right)
\end{aligned}
$$


donde, $x_{j} \in \mathrm{T}^{a_{j}}\left(\mathcal{L}_{T_{I}}^{m}(\Gamma)\right)$ para $1 \leq j \leq n, y, x_{0} \in$ $\mathrm{T}^{a_{0}}\left(\mathcal{L}_{T_{I}}^{m}(\Gamma)\right) \cup\left\{R_{\emptyset}\right\}$, con, $a_{0}=\left\langle a_{1}, \ldots, a_{n}\right\rangle, a_{0}, \ldots, a_{n} \in$ $T_{I}$. Por cierto recordar que $\mathcal{R}$ el $\left(\mathcal{O}_{I}\right)$ es definido en 3.7 item 3.

- Para $\alpha \in \mathfrak{F}_{q k}^{l+1}\left(\mathcal{L}_{T_{I}}^{m}(\Gamma)\right)$, con $1 \leq l<\omega$ :

a) Para $\alpha \equiv \neg\left(\alpha^{\prime}\right)$, donde $\alpha^{\prime} \in \mathfrak{F}_{q k}^{l}\left(\mathcal{L}_{T_{I}}^{m}(\Gamma)\right)$. Se tiene

$$
\Psi \models \alpha[v] \text {, si y solo si, no } \Psi \models \alpha^{\prime}[v] \text {. }
$$

b) Para $\alpha \equiv \forall^{j} x_{j}\left(\alpha^{\prime}\right)$, donde $\alpha^{\prime} \in \mathfrak{F}_{q k}^{l}\left(\mathcal{L}_{T_{I}}^{m}(\Gamma)\right), j \leq q$ :

$$
\begin{aligned}
& \text { Psi } \models \alpha[v] \text {, si y solo si, para todo } i \in j, \\
& \qquad p_{i} \in U^{a_{i}}(\Psi) \text {, entonces: } \Psi \models \alpha^{\prime}\left[v_{x_{j}}^{p_{j}}\right] .
\end{aligned}
$$

c) Para $\alpha \equiv \wedge_{j}\left(\alpha_{j}\right)$, donde $\alpha_{i} \in \mathfrak{F}_{q k}^{l}\left(\mathcal{L}_{T_{I}}^{m}(\Gamma)\right)$, con $i \in$ $j<k$ :

$$
\Psi \models \alpha[v], \text { si y solo si, para todo } i \in j, \Psi \models \alpha_{i}[v] \text {. }
$$

Si no se incluye el caso $\alpha \in \mathfrak{F}_{q k}^{l}\left(\mathcal{L}_{T_{I}}^{m}(\Gamma)\right)$, para $\alpha \in \mathfrak{F}_{q k}^{l+1}\left(\mathcal{L}_{T_{I}}^{m}(\Gamma)\right)$, es porque claramente cae en el paso inductivo anterior, haciendo innecesaria su definición.

Definición 3.9. Fijado $M$ modelo de $\Psi$ para $\mathcal{L}_{T_{I}}^{m}(\Gamma)$, y $\alpha \in \mathfrak{F}_{q k}\left(\mathcal{L}_{T_{I}}^{m}(\Gamma)\right)$. Definimos, $\Psi$ satisface la formula $\alpha$, si y solo si, para cualquier asignación de variable $v$ en $\Psi, \Psi \models \alpha[v]$. En simbolos, $\Psi \models \alpha$.

Proposición 3.10. Sea $\Psi$ una estructura, $\mathcal{L}_{T_{I}}^{m}(\Gamma)$ un lenguaje. Si se cumple

1. $\Psi$ permite $\mathcal{L}_{T_{I}}^{m}(\Gamma)$;

2. $\Gamma \subset U(\Psi)$;

3. Para todo $a \in T_{I}$, de modo que $\operatorname{ord}_{I}(a) \leq m$ entonces Const $_{a} \subset$ $U^{a}(\Psi)$.

Entonces podemos interpretar $\mathcal{L}_{T_{I}}^{m}(\Gamma)$ en $\Psi$. A los lenguajes que tengan tales características los llamaremos lenguaje canónico para $\Psi$. 
Demostración. Por la primera condición tenemos que estamos en condiciones de encontrar un modelo. Ahora veamos que $i_{\Gamma}$ la inclusión de $\Gamma$ a $U(\Psi)$, la cual podemos extender de manera simple a $\mathcal{C}_{\text {onst }} t_{T_{I}}^{m}$ agregando $i_{\Gamma}\left(R_{\emptyset}\right)=\emptyset^{\omega}$, cumple

1. Es claramente inyectiva.

2. Como $i_{\Gamma}\left[\right.$ Const $\left._{a}\right]=$ Const $_{a}$, es claro que usando la cuarta condición, tenemos la condición $b$ ) del ítem 2 de la definición 3.6.

3. Por construcción $i_{\Gamma}$, tenemos, $i_{\Gamma}\left(R_{\emptyset}\right)=\emptyset^{\omega}$.

En general para un lenguaje no hay mayor diferencia en escoger $R_{\emptyset}$ como $\emptyset^{\omega}$, puesto que en tal caso solo basta interpretar $\emptyset^{\omega}$ como él mismo. Por lo mismo y por la conveniencia de la notación fijaremos para lenguajes en general $R_{\emptyset}=\emptyset^{\omega}$.

\section{Transiciones entre estructuras.}

La noción a mostrar tiene una correspondencia al concepto de Equivalencia elemental mostrada en [8] pagina 55 .

Definición 3.11. Sean $\mathcal{L}_{T_{I}}^{m}(\Gamma)$ y $\mathcal{L}_{T_{I^{\prime}}}^{m^{\prime}}\left(\Gamma^{\prime}\right)$ lenguajes para los cuales hay un modelo en las estructuras $\Psi$ y $\Psi^{\prime}$ respectivamente. Decimos que una función $f$ de la forma

$$
\begin{aligned}
f: \mathfrak{F}_{q k}\left(\mathcal{L}_{T_{I}}^{m}(\Gamma)\right) \mapsto \mathfrak{F}_{q^{\prime} k^{\prime}}\left(\mathcal{L}_{T_{I^{\prime}}}^{m^{\prime}}\left(\Gamma^{\prime}\right)\right), \text { de modo que: } \\
\qquad \models \alpha \text {, si y solo si, } \Psi^{\prime} \models f(\alpha),
\end{aligned}
$$

es una transición de $\Psi$ en $\Psi^{\prime}$. Llamaremos al triple:

$\left(\left(q, q^{\prime}\right),\left(k, k^{\prime}\right),\left(m, m^{\prime}\right)\right)$ el argumento de la transición.

Por cierto si $f$ es una transición de $\Psi$ en $\Psi^{\prime}$ de argumento $\left(\left(q, q^{\prime}\right),\left(k, k^{\prime}\right),\left(m, m^{\prime}\right)\right), \mathrm{y}, g$ es una transición de $\Psi^{\prime}$ a $\Psi^{\prime \prime}$ de argumento $\left(\left(q^{\prime}, q^{\prime \prime}\right),\left(k^{\prime}, k^{\prime \prime}\right),\left(m^{\prime}, m^{\prime \prime}\right)\right)$. Entonces claramente $f \circ g$ es una transición de $\Psi$ a $\Psi^{\prime}$, y su argumento es de la forma $\left(\left(q, q^{\prime \prime}\right),\left(k, k^{\prime \prime}\right),\left(m, m^{\prime \prime}\right)\right)$.

En base al mismo ejemplo si $f$ es una transición de argumento $\left(\left(q, q^{\prime}\right),\left(k, k^{\prime}\right),\left(m, m^{\prime}\right)\right)$, y. $x=x^{\prime}$, para algún $x \in\{q, k, m\}$, entonces abreviaremos $\left(x, x^{\prime}\right)$ por $x$. 
Proposición 3.12. Sean $\mathcal{L}_{T_{I}}^{m}(\Gamma)$ un lenguaje, $\Psi$ una estructura. $M$ un modelo en $\Psi$ para $\mathcal{L}_{T_{I}}^{m}(\Gamma)$ y $\alpha \in \mathfrak{F}_{q k}\left(\mathcal{L}_{T_{I}}^{m}(\Gamma)\right)$. Se cumple:

1. El lenguaje ${ }^{27} \mathcal{L}_{T_{I}}^{m}(M[\Gamma])$, que cumple las condiciones

a) $M\left[\right.$ Const $\left._{a}\right]=$ Sim $_{a}^{\prime}$, donde $\mathcal{C o n s t}_{a}^{\prime}$ es el conjunto de constantes de tipo a para $\mathcal{L}_{T_{I}}^{m}(M[\Gamma])$.

b) $\mathcal{V} a r_{a}=\mathcal{V} a r_{a}^{\prime}$, donde $\mathcal{V} a r_{a}^{\prime}$ es el conjunto de variables de tipo a para $\mathcal{L}_{T_{I}}^{m}(M[\Gamma])$.

Es un lenguaje canónico para $\Psi$.

2. La función $\mathcal{C}$ an : $\mathfrak{F}_{q k}\left(\mathcal{L}_{T_{I}}^{m}(\Gamma)\right) \mapsto \mathfrak{F}_{q k}\left(\mathcal{L}_{T_{I}}^{m}(M[\Gamma])\right)$, de modo que $\mathcal{C} a n(\alpha)$ es el resultado de cambiar todas las apariciones de constantes $c$, en $\alpha$, por $M(c)$. Es una transición de $\Psi$ en $\Psi$ de argumento $(q, k, m)$.

Demostración. Enumerando las demostraciones:

1. Por la condición $b$ ) y el hecho de que $\Psi$ permite $\mathcal{L}_{T_{I}}^{m}(\Gamma)$, tenemos que $\Psi$ permite $\mathcal{L}_{T_{I}}^{m}(M[\Gamma])$. Claramente. por definición 3.6, $M[\Gamma] \subset U(\Psi) \cup\left\{\omega^{\emptyset}\right\}$, mas aún, $M\left[\right.$ Const $\left._{a}\right] \subset U^{a}(\Psi)$. Como $M\left[\right.$ Const $\left._{a}\right]=\mathcal{C}_{\text {onst }}^{\prime}$, luego $\mathcal{L}_{T_{I}}^{m}(M[\Gamma])$ cumple con las hipótesis de la proposición 3.10 , así $\mathcal{L}_{T_{I}}^{m}(M[\Gamma])$ es un lenguaje canónico para $\Psi$.

2. Sea $v$ una asignación de variable en $\Psi$ fija paro arbitraria. Para $\alpha \in \mathfrak{F}_{q k}\left(\mathcal{L}_{T_{I}}^{m}(\Gamma)\right)$, de modo que $\Psi \models \alpha[v]$, tenemos:

- O bien $\alpha \in \mathfrak{F}^{A}\left(\mathcal{L}_{T_{I}}^{m}(\Gamma)\right)$. En este caso

a) $\alpha \equiv x \stackrel{a}{=} y$, donde $a, y \in \mathrm{T}^{a}\left(\mathcal{L}_{T_{I}}^{m}(\Gamma)\right), \operatorname{ord}_{I}(a)<m$. Luego

$$
\Psi \models \alpha[v] \text {, si y solo si, } \Psi(x)[v]=\Psi(y)[v] .
$$

Si $x, y$ son variables tenemos que $\alpha=\mathcal{C}$ an $(\alpha)$, así

$$
\Psi \models \alpha[v], \text { si y solo si, } \Psi \models \mathcal{C} a n(\alpha)[v] .
$$

\footnotetext{
${ }^{27} M[$ ] es el operador imagen de $M$.
} 
Si ambas son constantes tenemos $M(x)=M(y)$, luego $\Psi(M(x))[v]=\Psi(M(y))[v]$, lo que equivale $\Psi \models$ $\operatorname{Can}(\alpha)[v]$. Por ultimo y sin perdida de generalidad si $x$ es un termino e $y$ una constante tenemos que $v(x)=M(y)$, lo que equivale $\Psi(x)[v]=\Psi(M(y))[v]$, es decir $\Psi=\mathcal{C}$ an $(\alpha)[v]$.

b) Si $\alpha \equiv x_{0}\left(x_{1}, \ldots, x_{1}\right)$, donde

$1 \leq j \leq n, x_{j} \in \mathrm{T}^{a_{j}}\left(\mathcal{L}_{T_{I}}^{m}(\Gamma)\right)$, y $x_{0} \in \mathrm{T}^{a_{0}}\left(\mathcal{L}_{T_{I}}^{m}(\Gamma)\right) \cup$ $\left\{R_{\emptyset}\right\}$ у $a_{0}=\left\langle a_{1}, \ldots, a_{n}\right\rangle, a_{0}, a_{1}, \ldots, a_{n} \in T_{I}^{28}$. Luego

$$
\begin{aligned}
& \Psi \models x_{0}\left(x_{1}, \ldots, x_{n}\right), \text { si y solo si, } \\
& \quad\left(\Psi\left(x_{1}\right)[v], \ldots, \Psi\left(x_{n}\right)[v]\right) \mathcal{R} \operatorname{Rel}\left(\mathcal{O}_{I}\right)\left(\Psi\left(x_{0}\right)[v]\right),
\end{aligned}
$$

Donde de manera provisoria usaremos:

$$
\delta(t)=\left\{\begin{array}{cl}
M(t) & , \text { si, } t \text { es una constante } \\
t & , \text { si, } t \text { es una variable. }
\end{array}\right.
$$

Como $\Psi(t)[v]=M(t)$ en caso de que $t$ sea constante, $\Psi(t)[v]=v(t)$ en caso de que $t$ sea variable. Entonces

$$
\begin{aligned}
& \Psi \models x_{0}\left(x_{1}, \ldots, x_{n}\right), \text { si y solo si, } \\
& \left(\Psi\left(\delta\left(x_{1}\right)\right)[v], \ldots, \Psi\left(\delta\left(x_{n}\right)\right)[v]\right) \operatorname{Rel}\left(\mathcal{O}_{I}\right)\left(\Psi\left(\delta\left(x_{0}\right)\right)[v]\right) .
\end{aligned}
$$

Notemos que por construcción

$\operatorname{Can}(\alpha)=\delta\left(x_{0}\right)\left(\delta\left(x_{1}\right), \ldots, \delta\left(x_{n}\right)\right)$, por lo tanto

$$
\Psi \models x_{0}\left(x_{1}, \ldots, x_{n}\right)[v] \text {, si y solo si, } \Psi=\mathcal{C} \text { an }(\alpha)[v] .
$$

- Para $\alpha \in \mathfrak{F}_{q k}^{l+1}(\Gamma)$, con $1 \leq l<\omega$,

a) Si $\alpha \equiv \neg\left(\alpha^{\prime}\right)$, donde $\alpha^{\prime} \in \mathfrak{F}_{q k}^{l}(\Gamma)$, luego por hipótesis de inducción

$$
\Psi \models \alpha^{\prime}[v], \text { si y solo si, } \Psi \models \mathcal{C} \text { an }\left(\alpha^{\prime}\right)[v] .
$$

Como $\mathcal{C}$ an $(\alpha)=\neg\left(\mathcal{C}\right.$ an $\left.\left(\alpha^{\prime}\right)\right)$, entonces, por definición 3.8

$$
\Psi \models \alpha[v] \text {, si y solo si, } \Psi \models \mathcal{C} a n(\alpha)[v] .
$$

\footnotetext{
${ }^{28}$ Ver definición 3.2 ítem 2 .
} 
b) Para $\alpha \equiv \forall^{j} x_{j}\left(\alpha^{\prime}\right)$, con $j<q$, al igual que el punto anterior, usando hipótesis de inducción tenemos

$$
\Psi \models \alpha^{\prime}[v], \text { si y solo si, } \Psi \models \mathcal{C} \text { an }\left(\alpha^{\prime}\right)[v] .
$$

Para $i \in j$, sea $x_{i}$ una variable de tipo $a_{i}$, sea $p_{i}$ un elemento arbitrario de $U^{a_{i}}(\Psi)$. Luego de la hipótesis de inducción

$$
\Psi \models \alpha^{\prime}\left[v_{x_{j}}^{p_{j}}\right] \text {, si y solo si, } \Psi \models \mathcal{C} \text { an }\left(\alpha^{\prime}\right)\left[v_{x_{j}}^{p_{j}}\right] .
$$

Luego por definición 3.8

$$
\Psi \models \alpha \text {, si y solo si, } \Psi \models \forall^{j} x_{j}\left(\mathcal{C} \text { an }\left(\alpha^{\prime}\right)\right)[v] .
$$

Como $\mathcal{C} a n(\alpha)=\forall^{j} x_{j}\left(\mathcal{C} a n\left(\alpha^{\prime}\right)\right)$, entonces

$$
\Psi \models \alpha \text {, si y solo si, } \Psi \models \mathcal{C} \text { an }(\alpha)[v] .
$$

c) Si $\alpha \equiv \wedge_{j} \alpha_{j}$, con $j<k$, donde $\alpha_{i} \in \mathfrak{F}_{q k}^{l}(\Gamma)$ para $i \in j$, luego por hipótesis de inducción

$$
\Psi \models \alpha_{i}[v], \text { si y solo si, } \Psi \models \mathcal{C} \text { an }\left(\alpha_{i}\right)[v],
$$

para $i \in j$. Luego por definición 3.8

$\Psi \models \alpha[v]$, si y solo si, para todo $i \in j, \Psi \models \mathcal{C} a n\left(\alpha_{i}\right)[v]$, Como $\mathcal{C} a n(\alpha) \equiv \wedge_{j}\left(\mathcal{C} a n\left(\alpha_{j}\right)\right)$, entonces por definición 3.8

$$
\Psi \models \alpha[v] \text {, si y solo si, } \Psi \models \mathcal{C} \text { an }(\alpha)[v] .
$$

Como $v$ y $\alpha$ son arbitrarios $\mathcal{C} a n$ es una transición de argumento $(q, k, m)$.

Ahora construimos los lenguajes pertinentes, para la demostración de reducción de individuos y de orden que realizaremos en este trabajo. Formalizando los comentarios sobre reducción de orden mostrado en el trabajo [1] página 8.

Definición 3.13. Sean $\Gamma$ un conjunto, $\mu$ una estructura múltiple, $e$ una estructura simple. Definimos:

1. Para $\mathcal{L}_{T_{I}}^{m}(\Gamma)$ lenguaje canónico para $\mu$, el lenguaje ${ }^{29} \mathcal{L}_{T}^{m}\left(\Gamma \cup \mathcal{A}_{\mu}\right)$,

\footnotetext{
${ }^{29}$ recordar $\mathcal{A}_{\mu}$ esta definido en 2.6.
} 
está dado por:

a) Para $\operatorname{ord}(a) \leq m$,

$$
\begin{aligned}
& \text { Const }_{a}^{\prime}= \\
& \bigcup\left\{\text { Const }_{b} \mid \mathcal{S}_{I}(b)=a\right\} \cup\left\{\delta_{\mu}^{T}(b) \mid\left\langle\mathcal{S}_{I}(b)\right\rangle=a \wedge \delta_{\mu}^{T}(b) \neq \emptyset^{\omega}\right\},
\end{aligned}
$$

donde Const $_{a}^{\prime}$ es el conjunto de constantes tipo a del lenguaje $\mathcal{L}_{T}^{m}\left(\Gamma \cup \mathcal{A}_{\mu}\right)$.

b) Para todo $a \in T_{I}, \operatorname{ord}_{I}(a) \leq m, \mathcal{V}_{a r}=\emptyset$, entonces $\operatorname{Var}_{\mathcal{S}_{I}(a)}=\emptyset$.

Llamaremos a tal lenguaje el lenguaje singular de $\mathcal{L}_{T_{I}}^{m}(\Gamma)$.

2. Para $\mathcal{L}_{T}^{m}(\Gamma)$ lenguaje canónico para e, el lenguaje ${ }^{30} \mathcal{L}_{T_{I_{T}}}^{1}\left(\Gamma_{\mathrm{I}} \cup \mathrm{I}_{e}\right)$ está dado por

a) $\Gamma_{\mathrm{I}}=\Gamma \times\{0\}$.

b) Para todo $a^{T}$ de modo que ord $I_{T}\left(a^{T}\right) \leq m$, fijamos el conjunto de constantes tipo $a^{T}$, como

$$
\text { Const }_{a^{T}}=\text { Const }_{a} \times\{0\} \text {. }
$$

c) Para todo $a \in T_{I_{T}}$, ord $I_{T}(a)=1$,

$$
\begin{aligned}
& \text { Const }_{a}= \\
& \left\{\delta_{e}^{\mathrm{I}}\left(a_{0}\right) \mid \delta_{e}^{\mathrm{I}}\left(a_{0}\right) \neq \emptyset \wedge a_{0}=\left\langle a_{1}, \ldots, a_{n}\right\rangle \wedge a=\left\langle a_{0}^{T}, a_{1}^{s}, \ldots, a_{n}^{s}\right\rangle\right\} .
\end{aligned}
$$

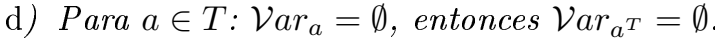

Llamaremos a tal lenguaje el lenguaje I fundamental de $\mathcal{L}_{T}^{m}(\Gamma)$.

Notemos que todas las clases definidas son subclases de la clase $\mathcal{E}_{I}(O b j)$ o $\mathcal{E}\left(O b j_{i}\right)$, dependiendo del caso. Como tales clases son conjuntos tenemos que las clases definidas también lo son.

Proposición 3.14. En términos de la definición anterior.

\footnotetext{
${ }^{30}$ recordar $I_{T}$ y $\mathrm{I}_{e}$ están definidos en 2.9 y 2.11 respectivamente.
} 
1. $\mathcal{L}_{T}^{m}\left(\Gamma \cup \mathcal{A}_{\mu}\right)$ es un lenguaje canónico para $\mu_{\mathcal{A}}$, la estructura análoga de $\mu$.

2. $\mathcal{L}_{T_{I_{T}}}^{1}\left(\Gamma_{\mathrm{I}} \cup \mathrm{I}_{e}\right)$ es un lenguaje canónico para $e_{\mathrm{I}}$, la estructura $\mathrm{I}$ fundamental de e.

Demostración. Enumerando las demostraciones:

1. Usando el hecho que $\mathcal{L}_{T_{I}}^{m}(\Gamma)$ es un lenguaje canónico para $\mu$ tenemos

$$
\Gamma \subset U(\mu) \text {, luego, } \Gamma \cup \mathcal{A}_{\mu} \subset U(\mu) \cup \mathcal{A}_{\mu},
$$

así, por la proposición 2.8 ítem $2 \Gamma \cup \mathcal{A}_{\mu} \subset U\left(\mu_{\mathcal{A}}\right)$. Claramente se cumple la segunda condición condición de la proposición 3.10. Con respecto a la tercera condición veamos que por hipótesis y proposición 2.8 ítem 4

$$
\text { Const }_{b} \subset U^{b}(\mu) \subset U^{\mathcal{S}_{I}(b)}\left(\mu_{\mathcal{A}}\right),
$$

luego tenemos que: para todo $a \in T$, con $\operatorname{ord}(a) \leq m$ :

$$
\bigcup\left\{\text { Const }_{b} \mid \mathcal{S}_{I}(b)=a\right\} \subset \bigcup\left\{U^{\mathcal{S}_{I}(b)}\left(\mu_{\mathcal{A}}\right) \mid \mathcal{S}_{I}(b)=a\right\}=U^{a}(\mu) .
$$

Por otro lado, para un $c$ arbitrario pero fijo, si ord $\left(\left\langle\mathcal{S}_{I}(c)\right\rangle\right)=$ ord $(a)$, entonces por la proposición 1.4 y 2.4 ítem 1, tenemos $\operatorname{ord}_{I}(c)+1=\operatorname{ord}(a)$. Claramente si $\operatorname{ord}(a)=0$ entonces

$$
\left\{\delta_{\mu}^{T}(b) \mid\left\langle\mathcal{S}_{I}(b)\right\rangle\right\}=\emptyset \subset U^{a}\left(\mu_{\mathcal{A}}\right) .
$$

Si $\operatorname{ord}(a) \leq 1$, veamos que

$$
\left\{\delta_{\mu}^{T}(b) \mid\left\langle\mathcal{S}_{I}(b)\right\rangle=a \wedge \delta_{\mu}^{T}(b) \neq \emptyset^{\omega}\right\} \subset \mathcal{A}_{\mu}^{l-1}
$$

donde $l=\operatorname{ord}_{I}(c)$. Veamos que por la proposición 2.5 ítem 3, $\delta_{\mu}^{T}(c)$ es de tipo $\left\langle\mathcal{S}_{I}(b)\right\rangle$, como $\left\langle\mathcal{S}_{I}(b)\right\rangle=a$, entonces, por la definición 1.10 y la proposición 2.5 ítem 2 y $3 \delta_{\mu}^{T}(c) \in \mathcal{O}\left(O b j_{i}\right)(a)$. Así, por la definición 2.6 y 1.9 ítem 2,

$$
\begin{gathered}
\left\{\delta_{\mu}^{T}(b) \mid\left\langle\mathcal{S}_{I}(b)\right\rangle=a \wedge \delta_{\mu}^{T}(b) \neq \emptyset^{\omega}\right\} \subset \mathcal{A}_{\mu}^{l-1} \cap \mathcal{O}\left(O b j_{i}\right)(a), \\
\mathcal{A}_{\mu}^{l-1} \cap \mathcal{O}\left(O b j_{i}\right)(a) \subset \mu_{\mathcal{A}}(l) \cap \mathcal{O}\left(O b j_{i}\right)(a)=U^{a}\left(\mu_{\mathcal{A}}\right) .
\end{gathered}
$$


En síntesis

$\bigcup\left\{\mathcal{C}_{\text {onst }} \mid \mathcal{S}_{I}(b)=a\right\} \cup\left\{\delta_{\mu}^{T}(b) \mid\left\langle\mathcal{S}_{I}(b)\right\rangle=a \wedge \delta_{\mu}^{T}(b) \neq \emptyset^{\omega}\right\} \subset U^{a}\left(\mu_{\mathcal{A}}\right)$,

que es equivalente, por la definición anterior ítem 1 parte $a$ ), Const $_{a}^{\prime} \subset U^{a}\left(\mu_{\mathcal{A}}\right)$. Por lo tanto se cumple la tercera condición de la proposición 3.10 .

La primera condición de 3.10 se deduce a partir de b) del ítem 1 de la definición anterior, para ello tengamos presente que para todo $a \in T_{I}, \operatorname{ord}_{I}(a) \leq m$ y $\mathcal{S}_{I}(a)=b$, tenemos, por proposición 2.8 ítem 4

$$
U^{b}\left(\mu_{\mathcal{A}}\right)=\emptyset \text {, entonces } U^{a}(\mu)=\emptyset .
$$

Como $\mu$ permite $\mathcal{L}_{T_{I}}^{m}(\Gamma)$, entonces

$$
U^{b}\left(\mu_{\mathcal{A}}\right)=\emptyset \text {, entonces, } \mathcal{V}_{a r_{a}}=\emptyset \text {. }
$$

Luego por el ítem $b$ ), ya que $a$ es arbitrario, tenemos

$$
U^{b}\left(\mu_{\mathcal{A}}\right)=\emptyset \text {, entonces } \mathcal{V} a r_{b}=\emptyset \text {. }
$$

Por lo tanto $\mathcal{L}_{T}^{m}\left(\Gamma \cup \mathcal{A}_{\mu}\right)$ es un lenguaje canónico para $\mu_{\mathcal{A}}$.

2. Ya que $\mathcal{L}_{T}^{m}(\Gamma)$ es un lenguaje canónico para $e \Gamma \subset U(e)$. Usando la proposición 2.12 ítem 3, y la definición anterior ítem 2

$$
(\Gamma \times\{0\}) \cup \mathrm{I}_{e} \subset(U(e) \times\{0\}) \cup \mathrm{I}_{e}=U\left(e_{\mathrm{I}}\right) .
$$

Lo que comprueba, dada la definición anterior, $\Gamma_{\mathrm{I}} \cup \mathrm{I}_{e} \subset U\left(e_{\mathrm{I}}\right)$, luego se cumple la condición 2 de la proposición 3.10.

Para la primera condición veamos que

$$
U^{a}(e)=\emptyset \text {, entonces, } \mathcal{V}_{a} r_{a}=\emptyset,
$$

así por la parte $d$ ) del ítem 2, de la definición anterior, tenemos

$$
U^{a}(e)=\emptyset \text {, entonces } \mathcal{V} a r_{a^{T}}=\emptyset .
$$

Usando la proposición 2.12 ítem 5 tenemos

$$
U^{a^{T}}\left(e_{\mathrm{I}}\right)=\emptyset \text {, entonces, } \mathcal{V} a r_{a^{T}}=\emptyset
$$

Manuscrito - Rev. Int. Fil., Campinas, v. 38, n.2, pp.89-154, jul.-ago. 2015. 
obteniendo así la primera condición de la proposición 3.10.

Para la tercera condición separaremos por el orden. Si ord $_{I_{T}}(a)=$ 0 , entonces $a=b^{s} \in I_{T}$. En tal caso notemos que, dado que $\mathcal{L}_{T}^{m}(\Gamma)$ es un lenguaje canónico para e y la definición anterior,

$$
\text { Const }_{a}=\text { Const }_{b} \times\{0\} \subset U^{b}(e) \times\{0\} .
$$

Luego por proposición 2.12 ítem 5 Const $_{a} \subset U^{a}\left(e_{I}\right)$. Para $\operatorname{ord}_{I_{T}}(a)=1$ tenemos por la definición anterior y 2.11 Const $_{a} \subset$ $\mathrm{I}_{e}$, además Const $_{a} \subset \mathcal{O}_{I_{T}}(\mathrm{Obj})(a)$ dado la proposición 2.12 y la definición anterior. Luego, por definición 1.9 ítem 2 y 2.11 ítem 4

$$
\text { Const }_{a} \subset \mathrm{I}_{e} \cap \mathcal{O}_{I_{T}}(O b j)(a)=U^{a}\left(e_{\mathrm{I}}\right) \text {. }
$$

Quedando claro que para todo $a \in T_{I_{T}}$, con $\operatorname{ord}_{I_{T}}(a) \leq 1$, Const $_{a} \subset U^{a}\left(e_{\mathrm{I}}\right)$. Por lo tanto $\mathcal{L}_{I_{T}}\left(\Gamma_{\mathrm{I}} \cup \mathrm{I}_{e}\right)$ es un lenguaje canónico para $e_{\mathrm{I}}$.

Antes de continuar notemos que si $\mathcal{V}_{a r} \neq \emptyset$ para algún $b$, con $\operatorname{ord}_{I}(b) \leq m$, tenemos que $\mathcal{V} a r_{\mathcal{S}_{I}(b)} \neq \emptyset$, dado la definición 3.13 ítem 1 parte $b)$. En este contexto veamos que si $\# I \leq \omega$, entonces para

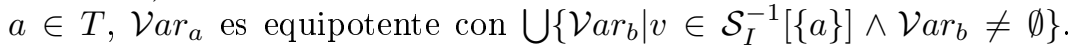
Este hecho está justificado por la proposición 2.4 ítem 3 y el hecho que la unión a lo mas numerable de numerables es numerable. Este sencillo hecho nos permite justificar la existencia de $u_{a}$, función biyectiva de $\bigcup\left\{\mathcal{V} a r_{b} \mid v \in \mathcal{S}_{I}^{-1}[\{a\}] \wedge \mathcal{V} a r_{b} \neq \emptyset\right\}$ a $\mathcal{V} a r_{a}$. Así definimos el operador $u$ como:

$$
u(t)=\left\{\begin{array}{cl}
\emptyset^{\omega} & , \text { si, } t=\emptyset^{\omega} \\
t & , \text { si, } t \in U^{a}(\mu), \text { para algún, } a \in T_{I} \\
u_{a}(t) & , \text { si, } t \in \bigcup\left\{\mathcal{V} a r_{b} \mid b \in \mathcal{S}_{I}^{-1}[\{a\}] \wedge \mathcal{V} a r_{b} \neq \emptyset\right\} .
\end{array}\right.
$$

Notemos que $u$ restringido a $\mathcal{V} a r_{T_{I}}^{m}$ es una biyección de $\mathcal{V}_{a} r_{T_{I}}^{m}$ a $\mathcal{V} a r_{T}^{m}$, puesto que cada función $u_{a}$ es una biyección. El motivo de lo anterior es que permitirá uno de los objetivos planteados en esta sección, a saber, la reducción de individuos. Pero con la condición de que $\# I \leq \omega$. 
Definición 3.15. Sean $\# I \leq \omega, \mathcal{L}_{T_{I}}^{m}(\Gamma)$ y $\mathcal{L}_{T}^{m}\left(\Gamma \cup \mathcal{A}_{\mu}\right)$ lenguajes canónicos para $\mu$ y $\mu_{\mathcal{A}}$ respectivamente. Definimos $\mathcal{U}$ de la forma

$$
\mathcal{U}: \mathfrak{F}_{q k}\left(\mathcal{L}_{T_{I}}^{m}(\Gamma)\right) \mapsto \mathfrak{F}_{q k}\left(\mathcal{L}_{T}^{m}\left(\Gamma \cup \mathcal{A}_{\mu}\right)\right),
$$

a través de los siguientes criterios

- Para $a \in \mathfrak{F}^{A}\left(\mathcal{L}_{T_{I}}^{m}(\Gamma)\right)$ :

1. Si $\alpha \equiv x \stackrel{a}{=} y$, donde $x, y \in \mathrm{T}^{a}\left(\mathcal{L}_{T_{I}}^{m}(\Gamma)\right), \operatorname{ord}_{I}(a)<m$, entonces

$$
\mathcal{U}(\alpha)=\left(\delta_{\mu}^{T}(a)(u(x)) \wedge \delta_{\mu}^{T}(a)(u(y))\right) \rightarrow u(x) \stackrel{\mathcal{S}_{I}(a)}{=} u(y) .
$$

2. Si $\alpha \equiv x_{0}\left(x_{1}, \ldots, x_{n}\right)$, donde, para todo $0 \leq j \leq n, x_{j} \in$ $\mathrm{T}^{a_{j}}\left(\mathcal{L}_{T_{I}}^{m}(\Gamma)\right), y, a_{0}=\left\langle a_{1}, \ldots, a_{n}\right\rangle$. Entonces

$$
\left.\mathcal{U}(\alpha)=\wedge_{n+1} \delta_{\mu}^{T}\left(a_{n+1}\right)\left(u\left(x_{n+1}\right)\right)\right) \rightarrow u\left(x_{0}\right)\left(u\left(x_{1}\right), \ldots, u\left(x_{n}\right)\right) .
$$

3. Si $\alpha \equiv x_{0}\left(x_{1}, \ldots, x_{n}\right)$, donde: para todo $1 \leq j \leq n, x_{j} \in$ $\mathrm{T}^{a_{j}}\left(\mathcal{L}_{T_{I}}^{m}(\Gamma)\right), y, x_{0}=\emptyset^{\omega}$, entonces

$$
\mathcal{U}(\alpha)=\emptyset^{\omega}\left(u\left(x_{1}\right), \ldots, u\left(x_{n}\right)\right) .
$$

- Para $\alpha \in \mathfrak{F}^{l+1}\left(\mathcal{L}_{T_{I}}^{m}(\Gamma)\right)$, donde $1 \leq l<\omega$, definimos por inducción

1. Si $\alpha \equiv \neg\left(\alpha^{\prime}\right)$, donde $\alpha^{\prime} \in \mathfrak{F}_{q k}^{l}\left(\mathcal{L}_{T_{I}}^{m}(\Gamma)\right)$, entonces

$$
\mathcal{U}(\alpha)=\neg\left(\mathcal{U}\left(\alpha^{\prime}\right)\right) .
$$

2. Si $\alpha \equiv \forall^{j} x_{j}\left(\alpha^{\prime}\right)$, donde $\alpha^{\prime} \in \mathfrak{F}_{q k}^{l}\left(\mathcal{L}_{T_{I}}^{m}(\Gamma)\right), y, 1 \leq j<q$. Entonces

$$
\mathcal{U}(\alpha)=\forall^{j} u\left(x_{j}\right)\left(\mathcal{U}\left(\alpha^{\prime}\right)\right) .
$$

3. Si $\alpha \equiv \wedge_{j} \alpha_{j}$ donde $\alpha_{i} \in \mathfrak{F}_{q k}^{l}\left(\mathcal{L}_{T_{I}}^{m}(\Gamma)\right)$ para $i \in j$. Entonces

$$
\mathcal{U}(\alpha)=\wedge_{j}\left(\mathcal{U}\left(\alpha_{j}\right)\right) .
$$

Por cierto recordar que $R_{\emptyset}=\emptyset^{\omega}$ según la convención acordada en el párrafo que precede a la definición 3.11. El motivo para recordar esto es el hecho que los $\delta_{\mu}^{T}(a)$ pueden ser $\emptyset^{\omega}$ en algunos casos. 
Definición 3.16. Sean $v$ y $v^{\prime}$ una asignación de variable en $\mu_{\mathcal{A}}$ y $\mu$ respectivamente, y sea $a \in T_{I}$, definimos: $v$ es reducible en $\mu$, si $y$ solo si existe $v^{\prime}$ asignación de variable en $\mu$ de modo que $v^{\prime}=v \circ u$.

Proposición 3.17. Sean $\# I \leq \omega, y, \alpha \in \mathfrak{F}^{A}\left(\mathcal{L}_{T_{I}}^{m}(\Gamma)\right)$. Se cumple

1. Si $v$ es reducible en $\mu$, entonces

$$
\mu \models \alpha\left[v^{\prime}\right] \text {, si y solo si, } \mu_{\mathcal{A}} \models \mathcal{U}(\alpha)[v] \text {. }
$$

Donde $v^{\prime}=v \circ u$ y $v^{\prime}$ una asignación de variable en $\mu$.

2. Para toda asignación $v^{\prime}$ en $\mu$, existe $v$ asignación reducible en $\mu$, de modo que $v^{\prime}=v \circ u$.

3. Si $v$ no es reducible en $\mu$ y $\mu \models \alpha$, entonces $\mu_{\mathcal{A}} \models \mathcal{U}(\alpha)[v]$.

Demostración. Enumerando las demostraciones:

1. Primero veamos que, para cualquier constante $t$ en el lenguaje,

$$
\mu(t)\left[v^{\prime}\right]=t=u(t)=\mu_{\mathcal{A}}(u(t))[v] .
$$

Además para cualquier variable $t$ de tipo a en el lenguaje $\mathcal{L}_{T_{I}}^{m}(\Gamma)$,

$$
\mu(t)\left[v^{\prime}\right]=v^{\prime}(t)=v(u(t))=\mu_{\mathcal{A}}(u(t))[v] .
$$

a) Para $\alpha \equiv x \stackrel{a}{=} y$. Veamos que si tenemos $\mu \models \alpha\left[v^{\prime}\right]$, entonces, por definiciones 1.7 ítem 3 y 2.6 ítem 1 y claro artificio lógico

$$
\begin{aligned}
\mu(x)\left[v^{\prime}\right], \mu(y)\left[v^{\prime}\right] \in \operatorname{Rel}(\mathcal{O})\left(\delta_{\mu}^{T}(a)\right), & \\
& \text { entonces, } \mu(x)\left[v^{\prime}\right]=\mu(y)\left[v^{\prime}\right] .
\end{aligned}
$$

Usando lo mostrado antes de empezar, tenemos

$$
\begin{aligned}
\mu_{\mathcal{A}}(u(x))[v], \mu_{\mathcal{A}}(u(y))[v] & \in \operatorname{Rel}(\mathcal{O})\left(\delta_{\mu}^{T}(a)\right), \\
& \text { entonces, } \mu_{\mathcal{A}}(u(x))[v]=\mu_{\mathcal{A}}(u(y))[v] .
\end{aligned}
$$

Manuscrito - Rev. Int. Fil., Campinas, v. 38, n.2, pp.89-154, jul.-ago. 2015. 
Por lo tanto, por definición $3.15, \mu \models \alpha\left[v^{\prime}\right]$, entonces, $\mu_{\mathcal{A}} \models$ $\mathcal{U}(\alpha)[v]$.

Por otro lado si $\mu_{\mathcal{A}} \models \mathcal{U}(\alpha)[v]$, entonces

$$
\begin{aligned}
& \mu_{\mathcal{A}}(u(x))[v], \mu_{\mathcal{A}}(u(y))[v] \in \operatorname{Rel}(\mathcal{O})\left(\delta_{\mu}^{T}(a)\right) \\
&, \text { entonces, } \mu_{\mathcal{A}}(u(x))[v]=\mu_{\mathcal{A}}(u(y))[v],
\end{aligned}
$$

tenemos, por la igualdad mostrada mas arriba y las definiciones 1.7 ítem 4 y 2.6 ítem 1

$$
\mu(x)\left[v^{\prime}\right], \mu(y)\left[v^{\prime}\right] \in U^{a}(\mu), \text { entonces, } \mu(x)\left[v^{\prime}\right]=\mu(y)\left[v^{\prime}\right] .
$$

Ahora bien, $x, y$ son términos de tipo $a$, luego, puestas: la definición 3.7, el hecho de que $\mathcal{L}_{T_{I}}^{m}(\Gamma)$ es un lenguaje canónico para $\mu$, el ítem 3 proposición 3.10 , tenemos que $\mu(x)\left[v^{\prime}\right], \mu(y)\left[v^{\prime}\right] \in U^{a}(\mu)$, así

$$
\mu(x)\left[v^{\prime}\right]=\mu(y)\left[v^{\prime}\right],
$$

luego por definición 3.8 tenemos $\mu=\alpha[v]$. Por lo tanto

$$
\mu \models \alpha\left[v^{\prime}\right] \text {, si y solo si, } \mu_{\mathcal{A}} \models \mathcal{U}(\alpha)[v] .
$$

b) Para $\alpha \equiv x_{0}\left(x_{1}, \ldots, x_{n}\right)$. Veamos que, usando el hecho $\mu(t)\left[v^{\prime}\right]=\mu_{\mathcal{A}}(u(t))[v]$ mostrado mas arriba,

$$
\left(\mu_{\mathcal{A}}\left(u\left(x_{1}\right)\right)[v], \ldots, \mu_{\mathcal{A}}\left(u\left(x_{n}\right)\right)[v]\right) \in \mathcal{R} \operatorname{Rel}(\mathcal{O})\left(\mu_{\mathcal{A}}\left(u\left(x_{0}\right)\right)[v]\right) .
$$

Usando un razonamiento tautológico tenemos: Para todo $j \in\{0, \ldots, n\}$,

$$
\begin{gathered}
\mu_{\mathcal{A}}\left(u\left(x_{j}\right)\right)[v] \in \mathcal{R} e l(O)\left(\delta_{\mu}^{T}\left(a_{j}\right)\right), \text { entonces, } \\
\left(\mu_{\mathcal{A}}\left(u\left(x_{1}\right)\right)[v], \ldots, \mu_{\mathcal{A}}\left(u\left(x_{n}\right)\right)[v]\right) \in \mathcal{R} \operatorname{Rel}(\mathcal{O})\left(\mu_{\mathcal{A}}\left(u\left(x_{0}\right)\right)[v]\right) .
\end{gathered}
$$

Luego, por la definición 3.8,

$$
\left.\mu_{\mathcal{A}}=\wedge_{n+1} \delta_{\mu}^{T}\left(a_{n+1}\right)\left(u\left(x_{n+1}\right)\right) \rightarrow u\left(x_{0}\right)\left(u\left(x_{1}\right), \ldots, u\left(x_{n}\right)\right)\right)[v] .
$$

Por otro lado, si tenemos $\mu_{\mathcal{A}}=\mathcal{U}(\alpha)[v]$, entonces para todo $j \in\{0, \ldots, n\}$,

$$
\mu_{\mathcal{A}}\left(u\left(x_{j}\right)\right)[v] \in \mathcal{R} \operatorname{Rel}(O)\left(\delta_{\mu}^{T}\left(a_{j}\right)\right), \text { entonces, }
$$




$$
\left(\mu_{\mathcal{A}}\left(u\left(x_{1}\right)\right)[v], \ldots, \mu_{\mathcal{A}}\left(u\left(x_{n}\right)\right)[v]\right) \in \operatorname{Rel}(\mathcal{O})\left(\mu_{\mathcal{A}}\left(u\left(x_{0}\right)\right)[v]\right) .
$$

De lo que deducimos, usando la igualdad $\mu(t)\left[v^{\prime}\right]=\mu_{\mathcal{A}}(u(t))[v]$ mostrada mas arriba:

Para todo $j \in\{0, \ldots, n\}$,

$$
\begin{gathered}
\mu\left(x_{j}\right)\left[v^{\prime}\right] \in \mathcal{R} \operatorname{Rel}(O)\left(\delta_{\mu}^{T}\left(a_{j}\right)\right), \text { entonces, } \\
\left(\mu\left(x_{1}\right)\left[v^{\prime}\right], \ldots, \mu\left(x_{n}\right)\left[v^{\prime}\right]\right) \in \mathcal{R} \operatorname{Rel}(\mathcal{O})\left(\mu\left(x_{0}\right)\left[v^{\prime}\right]\right) .
\end{gathered}
$$

Notemos que, para $x_{j}$ variable de tipo $a_{j}$, dada la definición 3.7, se cumple $v^{\prime}\left(x_{j}\right) \in U^{a}(\mu)$, es decir, dada la definición $3.8, \delta_{\mu}^{T}\left(a_{0}\right)\left(\mu\left(x_{0}\right)\left[v^{\prime}\right]\right)$. Ah, por cierto si $x_{j}$ es una constante, claramente $x_{j} \in U^{a}(\mu)$, puesto que, dada la proposición 2.11 item $4, x_{j} \in \mathcal{C}$ onst $_{a} \in U^{a}(\mu)$. Por lo tanto

$$
\left(\mu\left(x_{1}\right)\left[v^{\prime}\right], \ldots, \mu\left(x_{n}\right)\left[v^{\prime}\right]\right) \in \mathcal{R} \operatorname{Rel}(\mathcal{O})\left(\mu\left(x_{0}\right)\left[v^{\prime}\right]\right) .
$$

Así $\mu \models \alpha[v]$. En síntesis

$$
\mu \models \alpha\left[v^{\prime}\right] \text {, si y solo si, } \mu_{\mathcal{A}}=\mathcal{U}(\alpha)[v] .
$$

c) Para $\alpha \equiv \emptyset^{\omega}\left(x_{1}, \ldots, x_{n}\right)$, es claro que lo siguiente,

$$
\begin{aligned}
& \emptyset^{\omega}\left(\mu\left(x_{1}\right)\left[v^{\prime}\right], \ldots, \mu\left(x_{n}\right)\left[v^{\prime}\right]\right), \\
& \text { si y solo si, } \emptyset^{\omega}\left(\mu_{\mathcal{A}}\left(u\left(x_{1}\right)\right)[v], \ldots, \mu_{\mathcal{A}}\left(u\left(x_{n}\right)\right)[v]\right) .
\end{aligned}
$$

es un tautología. Es decir, $\mu_{\mathcal{A}} \models \mathcal{U}(\alpha)[v]$, si y solo si, $\mu \models$ $\alpha\left[v^{\prime}\right]$.

2. Sea $v^{\prime}$ una asignación de variable en $\mu$. Construimos, para $z \in$ $\mathcal{V} a r_{T}^{m}$

$$
v(z)=\left\{\begin{array}{cl}
v^{\prime}\left(u^{-1}(z)\right) & , \text { si, } z \in u\left[\mathcal{V} a r_{T_{I}}^{m}\right], \text { para algún } a \in T_{I} \\
v_{0}(z) & , \text { en otro caso. }
\end{array}\right.
$$

donde $u[]$ es el operador imagen de $u, v_{0}$ es otra asignación de variable en $\mu_{\mathcal{A}}$. Notemos que $u^{-1}$ esta bien definida por ser inyectiva. Además $v$ es una asignación de variable en $\mu_{\mathcal{A}}$, dada la proposición 2.8 ítem 4 y definición 3.7. Ahora veamos que

$$
v \circ u(x)=v^{\prime}\left(u^{-1}(u(x))=v^{\prime}(x)\right.
$$

para cualquier $x \in \mathcal{V} a r_{T_{I}}^{m}$, así $v^{\prime}=v \circ u$. 
3. Sea $v$, fija, una asignación no reducible en $\mu$ entonces existe $Z$ conjunto, no vacío, tal que, para toda $v^{\prime}$ asignación de variable en $\mu, x^{\prime} \in Z, x^{\prime} \in \mathcal{V}_{a r} r_{T_{I}}^{m}, v \circ u\left(x^{\prime}\right) \neq v^{\prime}\left(x^{\prime}\right)$. Ahora, dada la definición 3.1 , tenemos $x^{\prime} \in \mathcal{V}_{a} r_{a^{\prime}}$, para algún $a^{\prime}$, con $\operatorname{ord}_{I}\left(a^{\prime}\right)<m$. En este contexto, supongamos que $v\left(u\left(x^{\prime}\right)\right) \in U^{a}(\mu)$, y construyamos:

$$
v^{*}(z)=\left\{\begin{array}{cl}
v \circ u(z) & , \text { si, } z=x^{\prime} \\
v^{\prime \prime}(z) & , \text { en otro caso. }
\end{array}\right.
$$

donde $v^{\prime \prime}$ es una asignación de variable en $\mu$. Notemos que claramente $v^{*}$ es una asignación de variable en $\mu$ dado que $v^{*}\left(x^{\prime}\right) \in$ $U^{a}(\mu)$. Luego $v^{*}\left(x^{\prime}\right)=v \circ u\left(x^{\prime}\right)$, lo cual es una contradicción puesto que $v \circ u\left(x^{\prime}\right) \neq v^{\prime}\left(x^{\prime}\right)$ para cualquier asignación de variable, $v^{\prime}$, en $\mu$. Por lo tanto, para $x^{\prime} \in Z$, tenemos $v\left(u\left(x^{\prime}\right)\right) \notin U^{a}(\mu)$, lo cual demuestra lo propuesto, dada la definición 3.7. Ya desarrollado lo suficiente el concepto de no reducible en $\mu$, continuemos.

a) Si $\alpha \equiv x \stackrel{a}{=} y$, entonces, sin perdida de generalidad

1) O bien $x, y \notin Z$.

En este caso construyamos la asignación variable $v_{0}$ en $\mu_{\mathcal{A}}$, tal que

$$
v_{0}(z)=\left\{\begin{array}{cl}
v(z) & , \text { si, } z \neq u\left(x^{\prime}\right) \text { para } x^{\prime} \notin Z \\
v_{0}^{\prime}(z) & , \text { si, } z=u\left(x^{\prime}\right) \text { para } x^{\prime} \in Z
\end{array}\right.
$$

donde $v_{0}^{\prime}$ es reducible reducible en $\mu$, de modo que $v_{1}^{\prime}=$ $v_{0}^{\prime} \circ u$. Notemos que $v_{0}$ es una asignación de variable en $\mu_{\mathcal{A}}$, por el hecho de que $v_{0}^{\prime}$ y $v$ son asignaciones de variable en $\mu$. Para ver si es reducible, veamos que, para toda variable $x$ tal que $x \notin Z$, existe $v_{x}^{\prime}$ asignación de variable en $\mu$ de modo que $v_{x}^{\prime}(x)=v \circ u(x)$. Luego construimos la asignación de variable en $\mu$

$$
v_{1}(x)= \begin{cases}v_{x}^{\prime}(x) & , \text { si }, x \notin Z \\ v_{1}^{\prime}(x) & , \text { si }, x \in Z\end{cases}
$$

Notemos que $v_{1}$ es una asignación de variable en $\mu$, dado que, para cada $s \in \mathcal{V}_{a r}^{m}, s \notin Z$, $v_{s}$ es una asignación variable en $\mu$. Notemos además que

$$
v_{1}(s)=v_{s}^{\prime}(s)=v \circ u(s)=v_{0} \circ u(s) \text { para } s \notin Z .
$$

Manuscrito - Rev. Int. Fil., Campinas, v. 38, n.2, pp.89-154, jul.-ago. 2015. 


$$
v_{1}(s)=v_{1}^{\prime}(s)=v_{0} \circ u(s)=v_{0} \circ u(s) \text { para } s \in Z .
$$

Es decir $v_{1}=v_{0} \circ u$. Luego de haber mostrado esta construcción comprobemos que

$$
\mu_{\mathcal{A}}=\mathcal{U}(\alpha)[v] \text {, si y solo si, } \mu_{\mathcal{A}} \models \mathcal{U}(\alpha)\left[v_{0}\right],
$$

esto se debe a que, para $s \notin Z$,

$a^{\prime} \mathrm{O}$ bien, $s$ es una variable, $\mathrm{y}, v(u(s))=v_{0}(u(s))$.

Así, $\mu_{\mathcal{A}}(u(s))[v]=\mu_{\mathcal{A}}(u(s))\left[v_{0}\right]$.

$b^{\prime}$ O bien, $s$ es una constante, $\mathrm{y}, s=u(s)=\mu_{\mathcal{A}}(u(s))[v]=$ $\mu_{\mathcal{A}}(u(s))\left[v_{0}\right]$.

Luego por hipótesis $\mu \models \alpha\left[v_{1}\right]$, y por el ítem 1 de esta proposición, tenemos $\mu_{\mathcal{A}} \models \mathcal{U}(\alpha)\left[v_{0}\right]$. Luego $\mu_{\mathcal{A}} \models$ $\mathcal{U}(\alpha)[v]$

2) O bien $x \in Z$. En ese caso basta ver que $v(u(x)) \notin$ $U^{a}(\mu)$, dado que $v$ no es reducible en $\mu$. En ese caso $\mu_{\mathcal{A}}(u(x))[v] \notin \mathcal{R} \operatorname{el}\left(\delta_{\mu}^{T}(a)\right)$, lo cual hace que

$$
\begin{aligned}
\mu_{\mathcal{A}}(u(x))[v], \mu_{\mathcal{A}}(u(y))[v] & \in \operatorname{Rel}(\mathcal{O})\left(\delta_{\mu}^{T}(a)\right), \\
& \text { entonces, } \mu_{\mathcal{A}}(u(x))[v]=\mu_{\mathcal{A}}(u(y))[v],
\end{aligned}
$$

sea una clara tautología. Por lo tanto $\mu_{\mathcal{A}} \models \mathcal{U}(\alpha)[v]$.

b) Para $\alpha \equiv x_{0}\left(x_{1}, \ldots, x_{n}\right)$, sin perdida de generalidad

1) O bien $x_{0}, x_{1}, \ldots, x_{n} \notin Z$.

En este caso usaremos la construcción hecha en el punto anterior, veamos que,

$$
\begin{aligned}
& \mu_{\mathcal{A}} \models \\
& \wedge_{n+1}\left(\delta_{\mu}^{T}\left(a_{n+1}\right)\left(u\left(x_{n+1}\right)\right)\right) \rightarrow u\left(x_{0}\right)\left(u\left(x_{1}\right), \ldots, u\left(x_{n}\right)\right)[v], \\
& \text { equivale, } \\
& \quad \mu_{\mathcal{A}} \models \\
& \wedge_{n+1}\left(\delta_{\mu}^{T}\left(a_{n+1}\right)\left(u\left(x_{n+1}\right)\right)\right) \rightarrow u\left(x_{0}\right)\left(u\left(x_{1}\right), \ldots, u\left(x_{n}\right)\right)\left[v_{0}\right] .
\end{aligned}
$$


Esto se debe que para $x \notin Z$, entonces $\mu_{\mathcal{A}}(u(x))[v]=$ $\mu_{\mathcal{A}}(u(x))\left[v_{0}\right]$, cosa ya demostrada en el ítem anterior subsección $a$ ). Luego por hipótesis $\mu \models \alpha\left[v_{1}\right]$, y por el ítem 1 de esta proposición, tenemos

$$
\begin{aligned}
& \mu_{\mathcal{A}} \models \\
& \wedge_{n+1}\left(\delta_{\mu}^{T}\left(a_{n+1}\right)\left(u\left(x_{n+1}\right)\right)\right) \rightarrow u\left(x_{0}\right)\left(u\left(x_{1}\right), \ldots, u\left(x_{n}\right)\right)\left[v_{0}\right] . \\
& \text { Luego } \mu_{\mathcal{A}} \models \mathcal{U}(\alpha)[v]
\end{aligned}
$$

2) O bien existe $q \in\{0, \ldots, n\}$, de modo que $x_{q} \in Z$. En ese caso basta ver que $v\left(u\left(x_{q}\right)\right) \notin U^{a}(\mu)$, dado que $v$ no es reducible en $\mu$. En ese caso $\mu_{\mathcal{A}}\left(u\left(x_{q}\right)\right)[v] \notin$ $\operatorname{Rel}\left(\delta_{\mu}^{T}(a)\right)$, lo cual hace que

$$
\begin{aligned}
& \mu_{\mathcal{A}} \models \\
& \left(\wedge_{n+1}\left(\delta_{\mu}^{T}\left(a_{n+1}\right)\left(u\left(x_{n+1}\right)\right)\right) \rightarrow u\left(x_{0}\right)\left(u\left(x_{1}\right), \ldots, u\left(x_{n}\right)\right)\right)[v],
\end{aligned}
$$

sea una clara tautología. Por lo tanto $\mu_{\mathcal{A}}=\mathcal{U}(\alpha)[v]$.

Por lo tanto tenemos que $\mu \models \alpha$, entonces, $\mu_{\mathcal{A}} \models \mathcal{U}(\alpha)[v]$, para $v$ no reducible.

c) Si $\alpha \equiv x_{0}\left(x_{1}, \ldots, x_{n}\right)$, con $x_{j} \in \mathrm{T}^{a_{j}}\left(\mathcal{L}_{T_{I}}^{m}(\Gamma)\right)$, para $1 \leq j \leq$ $n, \mathrm{y}, x_{0}=\emptyset$. Entonces

$$
\mathcal{U}(\alpha)=\emptyset^{\omega}\left(u\left(x_{1}\right), \ldots, u\left(x_{n}\right)\right) .
$$

En este caso comprobamos que para cualquier asignación de variable $v$, incluyendo no reducibles en $\mu$, tenemos no $\mu_{\mathcal{A}} \models$ $\mathcal{U}(\alpha)[v]^{31}$, en especifico para $v$ no reducible $\mu$. Del mismo modo tenemos que no $\mu \models \alpha$. Ocupando un razonamiento tautológico, tenemos que: para toda $v$ no reducible,

$$
\text { no } \mu_{\mathcal{A}} \models \mathcal{U}(\alpha)[v] \text {, entonces, no } \mu \models \alpha \text {. }
$$

Usando leyes de la implicatoria, veamos que, para toda $v$ no reducible en $\mu$,

$$
\mu \models \alpha \text {, entonces, } \mu_{\mathcal{A}}=\mathcal{U}(\alpha)[v] .
$$

\footnotetext{
${ }^{31}$ Solo basta ver las definiciones 1.7 ítem 3 y la 3.8 para entender lo trivial del hecho.
}

Manuscrito - Rev. Int. Fil., Campinas, v. 38, n.2, pp.89-154, jul.-ago. 2015. 
Demostrando por completo este ítem.

Lema 3.18. Para $\alpha \in \mathfrak{F}^{A}\left(\mathcal{L}_{T_{I}}^{m}(\Gamma)\right)$

$$
\mu \models \alpha \text {, si y solo si, } \mu_{\mathcal{A}} \models \mathcal{U}(\alpha) \text {. }
$$

\section{Demostración.}

- De izquierda a derecha. Por la proposición 3.17 ítem 3 e hipótesis, $\mu_{\mathcal{A}} \mid=\mathcal{U}(\alpha)[v]$, para $v$ no reducible a $\mu$. Por otro lado, por definición 3.16 y proposición 3.17 ítem 1 e hipótesis, $\mu_{\mathcal{A}} \models \mathcal{U}(\alpha)[v]$ para $v$ reducible en $\mu$. Por lo tanto $\mu_{\mathcal{A}} \models \mathcal{U}(\alpha)$.

- De derecha a izquierda. Basta tomar $\mu_{\mathcal{A}}=\mathcal{U}(\alpha)[v]$, con $v$ reducible en $\mu$, para que usando la proposición 3.17 ítem 2 y definición 3.16 , obtener $\mu \models \alpha\left[v^{\prime}\right]$. Como $v^{\prime}$ es arbitrario, entonces $\mu \models \alpha$.

Teorema 3.19. $\mathcal{U}$ es una transición de $\mu$ en $\mu_{\mathcal{A}}$, de argumento $(q, k, m)$.

Demostración. Sea $\alpha \in \mathfrak{F}^{A}\left(\mathcal{L}_{T_{I}}^{m}(\Gamma)\right)$ tenemos por el resultado anterior

$$
\mu \models \alpha \text {, si y solo si, } \mu_{\mathcal{A}} \models \mathcal{U}(\alpha) .
$$

Sea $\alpha \in \mathfrak{F}_{q k}^{l+1}\left(\mathcal{L}_{T_{I}}^{m}(\Gamma)\right)$, con $l \in \mathbb{N}^{0}$. Veamos

1. Si $\mu=\alpha$, y, $\alpha \equiv \neg \alpha^{\prime}$, con $\alpha^{\prime} \in \mathfrak{F}_{q k}^{l}\left(\mathcal{L}_{T_{I}}^{m}(\Gamma)\right)$, tenemos que

$$
\begin{array}{r}
\mu \models \alpha \text {, si y solo si, para toda asignación de variable en } \mu, v^{\prime}, \\
\text { no } \mu \models \alpha^{\prime}\left[v^{\prime}\right],
\end{array}
$$

usando una hipótesis de inducción, dada la forma inductiva de $\mathfrak{F}_{q k}\left(\mathcal{L}_{T_{I}}^{m}(\Gamma)\right)$, tenemos

$$
\begin{array}{r}
\mu \models \alpha \text {, si y solo si, para toda asignación de variable en } \mu_{\mathcal{A}}, v, \\
\text { no } \mu_{\mathcal{A}} \models \neg\left(\mathcal{U}\left(\alpha^{\prime}\right)\right)[v] .
\end{array}
$$

Por lo tanto por definición $3.15 \mu \models \alpha$, si y solo si, $\mu_{\mathcal{A}} \models \mathcal{U}(\alpha)$. 
2. Si $\mu \models \alpha$, y, $\alpha \equiv \forall^{j} x_{j}\left(\alpha^{\prime}\right)$, con $\alpha^{\prime} \in \mathfrak{F}_{q k}^{l}\left(\mathcal{L}_{T_{I}}^{m}(\Gamma)\right)$, con $j<q$, tenemos que, para una sucesión $x_{j}$ de variables en $\mathcal{L}_{T_{I}}^{m}(\Gamma)$ y $p_{j}$ una sucesión de objetos en $U(\mu)$, que respetan sus tipos ${ }^{32}$,

$\mu \models \alpha$, si y solo si, para toda asignación de variable en $\mu, b$,

$$
\mu \models \alpha^{\prime}\left[b_{x_{j}}^{p_{j}}\right]
$$

usando una hipótesis de inducción, dada la forma inductiva de $\mathfrak{F}_{q k}\left(\mathcal{L}_{T_{I}}^{m}(\Gamma)\right)$, tenemos

$\mu \models \alpha$, si y solo si, para toda asignación de variable en $\mu_{\mathcal{A}}, v$,

$$
\mu \models \mathcal{U}\left(\alpha^{\prime}\right)\left[v_{u\left(x_{j}\right)}^{p_{j}}\right] .
$$

Por lo tanto, por las definiciones 3.8 y la $3.15, \mu \models \alpha$, si y solo si, $\mu_{\mathcal{A}}=\mathcal{U}(\alpha)$.

3. Si $\mu \models \alpha$, y, $\alpha \equiv \wedge_{j}\left(\alpha_{k}\right)$, con $\alpha_{i} \in \mathfrak{F}_{q k}^{l}\left(\mathcal{L}_{T_{I}}^{m}(\Gamma)\right)$, con $i \in j<k$, tenemos que

$\mu \models \alpha$, si y solo si, para toda asignación de variable en $\mu, v^{\prime}$,

$$
\text { para todo } i \in j, \mu \models \alpha_{i}\left[v^{\prime}\right] \text {, }
$$

por hipótesis de inducción

$\mu \models \alpha$, si y solo si, para toda asignación de variable en $\mu_{\mathcal{A}}, v$, para todo $i \in j, \mu=\mathcal{U}\left(\alpha_{i}\right)[v]$,

Por lo tanto por definición $3.15 \mu \models \alpha$, si y solo si, $\mu_{\mathcal{A}} \models \mathcal{U}(\alpha)$.

En suma de todo lo mostrado, por definición 3.11, $\mathcal{U}$ es una transición de argumento $(q, k, m)$.

La importancia de este teorema es demostrar el hecho de que cualquier estructura con mas de un individuo puede ser reducida a una de sólo uno. Salvo una condición, el hecho de que \#I sea numerable.

\footnotetext{
${ }^{32}$ Es decir si $x_{i}$ es de tipo $a_{i}$ entonces $p_{i} \in U^{a_{i}}(\mu)$.
} 
Tal hecho tiene cierto sentido, puesto que en un lenguaje cualquiera es posible construir la siguiente proposición $\wedge_{j} P_{j}\left(x_{1}^{a_{j}}\right)$, donde, $P_{j}\left(x_{1}^{a_{j}}\right)$ es una formula que contiene libre a la variable $x_{1}^{a_{j}}, j$ algún cardinal mayor que $\omega$ y $a_{j}$ una sucesión en $I$, distinta a pares, con $\# I>\omega$. El problema de esta formula es el hecho que posee una cantidad no numerable de variables y no es trivial encontrar una transición que preserve la idea esencial de verdad semántica que se ha construido. En síntesis si se quiere un interpretación mas o menos fiel, se tiene la intuición de que esta debiera diferenciar las variables libres.

Antes de continuar, hay que señalar que para los lenguajes $\mathcal{L}_{T}^{m}(\Gamma), \mathrm{y}$, $\mathcal{L}_{T_{I_{T}}}^{1}(\Gamma)$, los conjuntos de variables $\mathcal{V}_{a r_{a}}, \mathcal{V} a r_{a^{T}}$, para $a \in T, \mathrm{y}, a^{T} \in I_{T}$ respectivamente, son equipolentes, puesto que ambos son numerables. Por cierto si uno de ellos es vacío necesariamente el otro lo es, para ello tener en mente la definición 3.13 ítem 2 parte $d$ ). Ahora, denotemos por $i_{a}$, una biyección que hace posible la equipolencia, y del mismo modo que en la transición anterior definimos un operador:

$$
i^{T}(t)=\left\{\begin{array}{cl}
\emptyset^{\omega} & , \mathrm{si}, t=\emptyset^{\omega} \\
(t, 0) & , \mathrm{si}, t \in U^{a}(e), \text { para algún } a \in T \\
i_{a}(t) & , \mathrm{si}, t \in \mathcal{V} a r_{a}, \text { para algún } a \in T .
\end{array}\right.
$$

Ahora estamos en condiciones de definir lo siguiente.

Definición 3.20. Sea $T$ un conjunto de tipos de un solo individuo y sea $I_{T}$ la superación de $T . \mathcal{L}_{T}^{m}(\Gamma)$ y $\mathcal{L}_{T_{I_{T}}}^{1}\left(\Gamma_{\mathrm{I}} \cup \mathrm{I}_{e}\right)$ son lenguajes canónicos para e y $e_{\mathrm{I}}$ respectivamente. Definimos el operador I de la forma

$$
\mathrm{I}: \mathfrak{F}_{q k}\left(\mathcal{L}_{T}^{m}(\Gamma)\right) \mapsto \mathfrak{F}_{q k}\left(\mathcal{L}_{T_{I_{T}}}^{1}\left(\Gamma_{\mathrm{I}} \cup \mathrm{I}_{e}\right)\right)
$$

que cumple los siguientes criterios:

- Para $\alpha \in \mathfrak{F}^{A}\left(\mathcal{L}_{T}^{m}(\Gamma)\right)$ :

1. Si $\alpha \equiv x \stackrel{a}{=} y$ con $x, y \in \mathrm{T}^{a}\left(\mathcal{L}_{T}^{m}(\Gamma)\right), y, a \in T$, entonces

$$
\mathrm{I}(\alpha)=i^{T}(x) \stackrel{a^{T}}{=} i^{T}(y) .
$$

2. Si $\alpha \equiv x_{0}\left(x_{1}, \ldots, x_{n}\right)$ y $x_{j} \in \mathrm{T}^{a_{j}}\left(\mathcal{L}_{T}^{m}(\Gamma)\right)$ para $0 \leq j \leq n$ con $a_{0}=\left\langle a_{1}, \ldots, a_{n}\right\rangle$, entonces

$$
\mathrm{I}(\alpha)=\delta_{e}^{\mathrm{I}}\left(a_{0}\right)\left(i^{T}\left(x_{0}\right), i^{T}\left(x_{1}\right), \ldots, i^{T}\left(x_{n}\right)\right) .
$$


3. Si $\alpha \equiv \emptyset^{\omega}\left(x_{1}, \ldots, x_{n}\right)$ y $x_{j} \in \mathrm{T}^{a_{j}}\left(\mathcal{L}_{T}^{m}(\Gamma)\right)$ para $1 \leq j \leq n$, entonces

$$
\mathrm{I}(\alpha)=\emptyset^{\omega}\left(i^{T}\left(x_{1}\right), \ldots, i^{T}\left(x_{n}\right)\right) .
$$

- Para $\alpha \in \mathfrak{F}_{q k}^{l+1}\left(\mathcal{L}_{T}^{m}(\Gamma)\right)$, con $1 \leq l \leq \omega$, definimos por inducción,

1. Si $\alpha \equiv \neg\left(\alpha^{\prime}\right)$, con $\alpha^{\prime} \in \mathfrak{F}_{q k}^{l}\left(\mathcal{L}_{T}^{m}(\Gamma)\right)$, entonces

$$
\mathrm{I}(\alpha)=\neg\left(\mathrm{I}\left(\alpha^{\prime}\right)\right) .
$$

2. Si $\alpha \equiv \forall^{j} x_{j}\left(\mathrm{I}\left(\alpha^{\prime}\right)\right)$, con $\alpha^{\prime} \in \mathfrak{F}_{q k}^{l}\left(\mathcal{L}_{T}^{m}(\Gamma)\right)$ y $j<q$, entonces

$$
\mathrm{I}(\alpha)=\forall^{j} i^{T}\left(x_{j}\right)\left(\mathrm{I}\left(\alpha^{\prime}\right)\right) .
$$

3. Si $\alpha \equiv \wedge_{j} \alpha_{j}$, con $\alpha_{i} \in \mathfrak{F}_{q k}^{l}\left(\mathcal{L}_{T}^{m}(\Gamma)\right)$ para $1 \leq i \leq j<k$, entonces

$$
\mathrm{I}(\alpha)=\wedge_{j}\left(\mathrm{I}\left(\alpha_{j}\right)\right) .
$$

Llamaremos a esta función el reductor I fundamental.

Lo que se ha querido hacer en esta definición es mostrar transición que permitirá la igualdad semántica entre las estructuras e y $e_{\mathrm{I}}$, la primera la estructura simple ${ }^{33}$ y la segunda la estructura I fundamental ${ }^{34}$

Lema 3.21. Se cumplen las siguientes afirmaciones

1. Sea $v$ una asignación de variable en $e, y, x \in \mathcal{V}_{a} r_{a}$ con $a \in$ $T$, entonces existe $v^{\prime}$ asignación de variable en $e_{I}$, de modo que $(v(x), 0)=v^{\prime} \circ i^{T}(x)$.

2. Sea $v^{\prime}$ una asignación de variable en $e_{\mathrm{I}}$, entonces existe $v$ una asignación de variable en e, tal que $(v(x), 0)=v^{\prime} \circ i^{T}(x)$, para $x \in \mathcal{V}_{a} r_{a}$ con $a \in T$.

Demostración. Enumerando las demostraciones:

\footnotetext{
${ }^{33}$ Ver definición 2.1 ítem 1.

${ }^{34}$ Ver definición 2.11 .
}

Manuscrito - Rev. Int. Fil., Campinas, v. 38, n.2, pp.89-154, jul.-ago. 2015. 
1. Para $v$ asignación de variable en $e$, podemos construir la siguiente valuación en $e_{\mathrm{I}}$

$$
v^{\prime}(t)=\left(v \circ i_{a}^{-1}(t), 0\right), \text { para } t \in \mathcal{V} a r_{a^{T}}, \text { para algún } a \in T
$$

que es una valuación de variable puesto que

$$
\mathcal{V} a r_{T_{I_{T}}}^{1}=\bigcup\left\{\mathcal{V} a r_{b} \mid \operatorname{ord}_{I_{T}}(b)<1\right\}=\bigcup\left\{{\left.\mathcal{V} a r_{a^{T}} \mid a \in T\right\}}\right.
$$

Además de

$$
v^{\prime}(t) \subset U^{a}(e) \times 0=U^{a^{T}}\left(e_{\mathrm{I}}\right),
$$

dada la proposición 2.12 ítem 5 . Ahora notemos que para $x$ variable de tipo $a$

$$
v^{\prime}\left(i_{a}(x)\right)=\left(v \circ i_{a}^{-1}\left(i_{a}(x)\right), 0\right)=(v(x), 0) .
$$

2. Para $v^{\prime}$ una asignación de variables en $e_{\mathrm{I}}$, definamos

$$
v(x)=X, \mathrm{si}, v^{\prime}\left(i^{T}(x)\right)=(X, 0)
$$

Notemos que si $x$ es una variable de tipo $a$ tenemos $v^{\prime}\left(i^{T}(x)\right) \in$ $U^{a^{T}}\left(e_{\mathrm{I}}\right)$. Luego, por la proposición 2.12 ítem 5 ,

$$
v^{\prime}\left(i^{T}(x)\right)=(v(x), 0) \in U^{a^{T}}\left(e_{\mathrm{I}}\right)=U^{a}(e) \times\{0\},
$$

así $v(x) \in U^{a}(e)$. Luego $v$ es una asignación de variable en $e$. Por ultimo notemos que por definición de $v$, tenemos

$$
(v(x), 0)=v^{\prime}\left(i^{T}(x)\right)=v^{\prime} \circ i^{T}(x) .
$$

Teorema 3.22. I es una transición de e en $e_{\mathrm{I}}$ de argumento $(q, k,(m, 1))$.

Demostración. Sea $v$ una asignación de variable en $v$ fija pero arbitraria. Primero demostraremos que

$$
e \models \alpha[v] \text {, si y solo si, } e_{\mathrm{I}}=\mathrm{I}(\alpha)\left[v^{\prime}\right],
$$

donde $(v(x), 0)=v^{\prime} \circ i^{T}(x)$ para $x \in \mathcal{V} a r_{a}$ con $a \in T$. 
- Notemos que para $t$ una variable de tipo $a$, tenemos por definición $3.8 e(t)[v]=v(t)$, usando que $(v(x), 0)=v^{\prime} \circ i^{T}(x)$, para $x \in \mathcal{V} a r_{a}$ con $a \in T$. Tenemos

$$
(e(t)[v], 0)=(v(t), 0)=v^{\prime} \circ i^{T}(t)=e_{\mathrm{I}}\left(i^{T}(t)\right)\left[v^{\prime}\right] .
$$

Por otro lado Notemos que si $t \in$ Const $_{a}$, para $a \in T$, ocurre, dada la proposición 2.12 ítem 5 y 3.14 ítem 2, además de la definición 3.13 ,

$$
(e(t)[v], 0)=(t, 0)=i^{T}(t)=e_{\mathrm{I}}\left(i^{T}(t)\right)\left[v^{\prime}\right], \mathrm{y}, i^{T}(t) \in \text { Const }_{a^{T}} .
$$

Por lo tanto tenemos que: si $t \in \mathrm{T}\left(\mathcal{L}_{T}^{m}(\Gamma)\right)$, entonces, $(e(t)[v], 0)=$ $e_{\mathrm{I}}\left(i^{T}(t)\right)\left[v^{\prime}\right]$.

- Para $\alpha \in \mathfrak{F}^{A}\left(\mathcal{L}_{T}^{m}(\Gamma)\right)$, veamos:

1. Si $\alpha \equiv x \stackrel{a}{=} y$, con $x, y \in \mathrm{T}^{a}\left(\mathcal{L}_{T}^{m}(\Gamma)\right)$, tenemos

$$
e=\alpha[v] \text {, si y solo si, } e(x)[v]=e(y)[v],
$$

por el ítem anterior, tenemos además

$$
e=\alpha[v] \text {, si y solo si, } e_{\mathrm{I}}\left(i^{T}(x)\right)\left[v^{\prime}\right]=e_{\mathrm{I}}\left(i^{T}(y)\right)\left[v^{\prime}\right] .
$$

Como $i^{T}(x), i^{T}(y) \in \mathrm{T}^{a^{T}}\left(\mathcal{L}_{T_{I_{T}}}^{1}\left(\Gamma_{\mathrm{I}} \cup \mathrm{I}_{e}\right)\right)$, dada la definición 3.1 y 3.13 , entonces, por definiciones 3.8 y 3.20 ,

$$
e \models \alpha[v] \text {, si y solo si, } e_{\mathrm{I}}=\mathrm{I}(\alpha)\left[v^{\prime}\right] .
$$

2. Para $\alpha \equiv x_{0}\left(x_{1}, \ldots, x_{n}\right)$, con $x_{j} \in \mathrm{T}^{a_{j}}\left(\mathcal{L}_{T}^{m}(\Gamma)\right), 0 \leq j \leq n$, y $a_{0}=\left\langle a_{1}, \ldots, a_{n}\right\rangle$, tenemos

$$
\begin{aligned}
& e=\alpha[v] \text {, si y solo si, } \\
& \qquad\left(e\left(x_{1}\right)[v], \ldots, e\left(x_{n}\right)[v]\right) \in \operatorname{Re} \operatorname{Ro}(\mathcal{O})\left(e\left(x_{0}[v]\right)\right) .
\end{aligned}
$$

Luego, usando la definición 3.10 ítem 1 y 2, además de el item anterior,

$$
\begin{aligned}
& e=\alpha[v], \text { si y solo si, } \\
& \left(e_{\mathrm{I}}\left(i^{T}\left(x_{0}\right)\right)\left[v^{\prime}\right], e_{\mathrm{I}}\left(i^{T}\left(x_{1}\right)\right)\left[v^{\prime}\right], \ldots, e_{\mathrm{I}}\left(i^{T}\left(x_{n}\right)\right)\left[v^{\prime}\right]\right) \in \mathrm{I}_{e}(a),
\end{aligned}
$$


donde $a=\left\langle a_{0}^{s}, a_{1}^{s}, \ldots, a_{n}^{s}\right\rangle$. Lo anterior equivale a

$$
\begin{aligned}
& e \models \alpha[v], \text { si y solo si, } \\
&\left(e_{\mathrm{I}}\left(i^{T}\left(x_{0}\right)\right)\left[v^{\prime}\right], e_{\mathrm{I}}\left(i^{T}\left(x_{1}\right)\right)\left[v^{\prime}\right], \ldots, e_{\mathrm{I}}\left(i^{T}\left(x_{n}\right)\right)\left[v^{\prime}\right]\right) \in \mathcal{R} e l\left(O_{I_{T}}\right)\left(\delta_{e}^{\mathrm{I}}(a)\right)
\end{aligned}
$$

dadas las definiciones 1.7 ítem 3, y, 2.11 ítem 1 y 2 . Por lo tanto, por las definiciones 3.8 y 3.20 ,

$$
e=\alpha[v] \text {, si y solo si, } e_{\mathrm{I}}=\mathrm{I}(\alpha)\left[v^{\prime}\right] .
$$

3. Si $\alpha \equiv \emptyset^{\omega}\left(x_{1}, \ldots, x_{n}\right)$, con $x_{j} \in \mathrm{T}^{a_{j}}\left(\mathcal{L}_{T}^{m}(\Gamma)\right), 1 \leq j \leq n$, entonces, claramente

$$
\begin{aligned}
\emptyset^{\omega}\left(e\left(x_{1}\right)[v], \ldots, e\left(x_{n}\right)[v]\right), \\
\text { si y solo si, } \emptyset^{\omega}\left(e_{\mathrm{I}}\left(x_{1}\right)\left[v^{\prime}\right], \ldots, e_{\mathrm{I}}\left(x_{n}\right)\left[v^{\prime}\right]\right),
\end{aligned}
$$

es una tautología. Luego de manera obvia

$$
e \models \alpha[v], \text { si y solo si, } e_{\mathrm{I}}=\mathrm{I}(\alpha)\left[v^{\prime}\right] .
$$

- Para $\alpha \in \mathfrak{F}_{q k}^{l+1}\left(\mathcal{L}_{T}^{m}(\Gamma)\right)$ con $l \in \mathbb{N}^{0}$. Veamos:

1. Para $\alpha \equiv \neg\left(\alpha^{\prime}\right)$ con $\alpha^{\prime} \in \mathfrak{F}_{q k}^{l}\left(\mathcal{L}_{T}^{m}(\Gamma)\right)$. Veamos que usando una hipótesis de inducción sobre la forma inductiva de $\mathfrak{F}_{q k}\left(\mathcal{L}_{T}^{m}(\Gamma)\right)$, tenemos

$$
e=\alpha^{\prime}[v], \text { si y solo si, } e_{\mathrm{I}} \models \mathrm{I}\left(\alpha^{\prime}\right)[v],
$$

luego, por definición 3.8

$$
e=\neg\left(\alpha^{\prime}\right)[v] \text {, si y solo si, } e_{\mathrm{I}} \models \neg\left(\mathrm{I}\left(\alpha^{\prime}\right)\right)[v],
$$

luego por definición 3.20

$$
e \models \alpha[v], \text { si y solo si, } e_{\mathrm{I}} \models \mathrm{I}(\alpha)[v] .
$$

2. Para $\alpha \equiv \forall^{j} x_{j}\left(\alpha^{\prime}\right)$ con $\alpha^{\prime} \in \mathfrak{F}_{q k}^{l}\left(\mathcal{L}_{T}^{m}(\Gamma)\right)$ y $j<q$. Veamos que usando la misma hipótesis de inducción usada y alguna que otra modificación conveniente

$$
e \models \alpha^{\prime}\left[v_{x_{j}}^{p_{j}}\right] \text {, si y solo si, } e_{\mathrm{I}}=\mathrm{I}\left(\alpha^{\prime}\right)\left[{v^{\prime}}_{i^{T}\left(x_{j}\right)}^{i^{T}\left(p_{j}\right)}\right],
$$


donde, $p_{i} \in U^{a}(e)$ para $i \in j$. Por cierto no es difícil ver que

$$
\left(v_{x_{j}}^{p_{j}}(x), 0\right)={v^{\prime}}_{i^{T}\left(x_{j}\right)}\left(i^{T}(x)\right), \text { para, } x \in \mathcal{V} a r_{a}, \text { con } a \in T,
$$

cosa que es necesaria para la hipótesis de inducción. Así tenemos, por definición 3.8 y 3.11

$$
e \models \alpha[v], \text { si y solo si, } e_{\mathrm{I}}=\forall^{j} i^{T}\left(x_{j}\right)\left(\mathrm{I}\left(\alpha^{\prime}\right)\right)[v] .
$$

Por lo tanto, puesta la definición 3.20

$$
e \models \alpha[v] \text {, si y solo si, } e_{\mathrm{I}}=\mathrm{I}(\alpha)\left[v^{\prime}\right] .
$$

3. Para $\alpha \equiv \wedge_{j} \alpha_{j}$ con $\alpha_{j} \in \mathfrak{F}_{q k}^{l}\left(\mathcal{L}_{T}^{m}(\Gamma)\right)$ y $i \in j<k$. Usando la hipótesis de inducción invocadas en los ítem anteriores

$$
e \models \alpha_{i}[v] \text {, si y solo si, } e_{\mathrm{I}}=\mathrm{I}\left(\alpha_{i}\right)\left[v^{\prime}\right],
$$

para $i \in j$. Como $i$ es arbitrario, por definición 3.8,

$$
e=\wedge_{j} \alpha_{j}[v], \text { si y solo si, } e_{\mathrm{I}}=\wedge_{j} \mathrm{I}\left(\alpha_{j}\right)\left[v^{\prime}\right],
$$

lo que equivale, puesta la definición 3.20,

$$
e \models \alpha[v] \text {, si y solo si, } e_{\mathrm{I}}=\mathrm{I}(\alpha)\left[v^{\prime}\right] .
$$

Ya habiendo demostrado lo propuesto, prosigamos. Supongamos que $e \models \alpha$, en tal caso tenemos que para cualquier $v, e \models \alpha[v]$. Por el lema 3.21 ítem 2 podemos tomar $v$ de modo que $(v(x), 0)=v^{\prime} \circ i^{T}(x)$, con $x \in \mathcal{V} a r_{T}^{m}$, para $v^{\prime}$ una asignación de variable $v^{\prime}$ arbitraria. Por lo tanto, por lo demostrado, $e_{\mathrm{I}}=\mathrm{I}(\alpha)\left[v^{\prime}\right]$, es decir, dado que $v^{\prime}$ es arbitraria, $e_{\mathrm{I}}=\mathrm{I}(\alpha)\left[v^{\prime}\right]$.

Por otro lado, si para cualquier asignación de variable $v^{\prime}$ en $e_{\mathrm{I}}$ tenemos $e_{\mathrm{I}}=\mathrm{I}(\alpha)\left[v^{\prime}\right]$, entonces, por lema 3.21 ítem 1 , para cualquier $v$ asignación de variable en $e$, puedo escoger $v^{\prime}$ de modo que $(v(x), 0)=v^{\prime} \circ i^{T}(x)$, $\operatorname{con} x \in \mathcal{V}_{a r}^{m}$. Por lo tanto, por lo demostrado tenemos $e=\alpha[v]$. Como $v$ es cualquiera $e \models \alpha$. Por lo tanto, y en un gran esfuerzo de síntesis, I es una transición de argumento $(q, k,(m, 1))$. 
Lo importante de este teorema es que nos muestra una manera de reducir estructuras usuales de un solo individuo, pero de orden arbitrario, a estructuras de orden 1, con la salvedad de tener varios individuos.

Antes de continuar quisiera mencionar que $T$ es numerable, dado que $\mathcal{G}^{(l)}(\{i\})$ es numerable ${ }^{35}$ para todo $1 \leq l<\omega$, luego como la unión numerable de numerables es numerables entonces $T$ es numerable, del mismo $I_{T}$ también lo es. El próximo corolario asume tal hecho.

Corolario 3.23. Sea $\mathcal{L}_{T_{I}}^{m}(\Gamma)$, de modo que $\# I \leq \omega$, un lenguaje canónico para $\mu$. Luego existe una transición de $\mu$ en $\left(\left(\mu_{\mathcal{A}}\right)_{\mathrm{I}}\right)_{\mathcal{A}}$, de argumento $(q, k,(m, 1))$.

Demostración. Usando $\mathcal{U} \circ \mathrm{I} \circ \mathcal{U}$, función desde $\mathcal{L}_{T_{I}}^{m}(\Gamma)$ a $\mathcal{L}_{T}^{1}\left(\left(\left(\Gamma \cup \mathcal{A}_{\mu}\right)_{\mathrm{I}} \cup\right.\right.$ $\left.\left.\mathrm{I}_{\mu_{\mathcal{A}}}\right) \cup \mathcal{A}_{\left(\mu_{\mathcal{A}}\right)_{\mathrm{I}}}\right)$, tenemos lo querido. Esto es debido a los teoremas $3.19 \mathrm{y}$ 3.22 , el hecho de que la composición de transiciones es una transición, y que $I_{T}$ es numerable.

Los teoremas 3.22 y corolario 3.23 corresponden a nuestra versión de reducción a orden 1, mostrada en el trabajo Definability and Invariance[1]. Para una estructura usual de Newton da Costa, que en nuestro caso corresponde a la estructura simple $e$, solo basta usar $I \circ \mathcal{U}$, para obtener una reducción correcta a lenguajes de primer orden.

Por cierto si alguien quiere saber mas sobre el caso \#I> $>$ ver [7], en especifico desde 2.25 hasta 2.33 .

\section{Referencias}

[1] DA COSTA, N. C. A.; RODRIGUES, A. A. M. Definability and Invariance, Studia Logica 86 (2007), no. 1, 1-30.

[2] ——. preprint. Remarks in Abstract Galois Theory, 2010.

[3] —_. ; BUENO, O. Remarks in Abstract Galois Theory, Manuscrito - Rev. Int. Fil., Campinas, v. 34, n.1, p 153-185, jan-jun. 2011.

\footnotetext{
${ }^{35}$ No es mas que comprobar que es equipolente con un producto cartesiano finito de elementos numerables, aunque la demostración en detalle es por inducción sobre $l$.
} 
[4] CASTILlO, C. I. Lógica y teoría de conjuntos, Universidad de Valencia auto-edición 2011, http://www.uv.es/ivorra/Libros/Logica.pdf.

[5] LANG, S. Algebra, Springer

[6] BELL, J.L. y SLOMSON, A.B. Models and Ultraproducts, NothHolland Publishing Company - 1969.

[7] NAVARRO, V. Estructuras de Newton Da Costa y Aplicaciones, tesis de magíster universidad de santiago, 2013.

[8] ROBINSON, A. Introducction to model theory and to the metamathematics of algebra, North-Holland Publishing Company - Amsterdam London 1974. 